LAWRENCE LIVERMORE N A T IO NAL LABORATORY
Laser Ion Acceleration from the Interaction of Ultra-Intense laser Pulse with thi foils

Matthew Allen

March 25, 2004 
This document was prepared as an account of work sponsored by an agency of the United States Government. Neither the United States Government nor the University of California nor any of their employees, makes any warranty, express or implied, or assumes any legal liability or responsibility for the accuracy, completeness, or usefulness of any information, apparatus, product, or process disclosed, or represents that its use would not infringe privately owned rights. Reference herein to any specific commercial product, process, or service by trade name, trademark, manufacturer, or otherwise, does not necessarily constitute or imply its endorsement, recommendation, or favoring by the United States Government or the University of California. The views and opinions of authors expressed herein do not necessarily state or reflect those of the United States Government or the University of California, and shall not be used for advertising or product endorsement purposes.

This work was performed under the auspices of the U.S. Department of Energy by University of California, Lawrence Livermore National Laboratory under Contract W-7405-Eng-48. 


\title{
Ion Acceleration from the Interaction of Ultra-Intense Lasers with Solid Foils
}

\author{
by \\ Matthew Mark Allen \\ B.S. (University of Rochester, Rochester, NY) 1997 \\ A dissertation submitted in partial satisfaction of the \\ requirements for the degree of \\ Doctor of Philosophy \\ in \\ Engineering-Nuclear Engineering \\ in the \\ GRADUATE DIVISION \\ of the \\ UNIVERSITY OF CALIFORNIA, BERKELEY
}

Committee in charge:

Edward Morse, Chair

Pravesh Patel

Donald Olander

Jonathan Wurtele

Spring 2004 
The dissertation of Matthew Mark Allen is approved:

\begin{tabular}{lc}
\hline Chair & Date \\
\hline & Date \\
\hline & \\
\hline 2 Date \\
\end{tabular}

University of California, Berkeley

Spring 2004 
Ion Acceleration from the Interaction

of Ultra-Intense Lasers with Solid Foils

Copyright 2004

by

Matthew Mark Allen 


\begin{abstract}
Ion Acceleration from the Interaction

of Ultra-Intense Lasers with Solid Foils
\end{abstract}

by

Matthew Mark Allen

Doctor of Philosophy in Engineering-Nuclear Engineering

University of California, Berkeley

Edward Morse, Chair

The discovery that ultra-intense laser pulses $\left(I>10^{18} \mathrm{~W} / \mathrm{cm}^{2}\right)$ can produce short pulse, high energy proton beams has renewed interest in the fundamental mechanisms that govern particle acceleration from laser-solid interactions. Experiments have shown that protons present as hydrocarbon contaminants on laser targets can be accelerated up to energies $>50 \mathrm{MeV}$.

Different theoretical models that explain the observed results have been proposed. One model describes a front-surface acceleration mechanism based on the ponderomotive potential of the laser pulse. At high intensities $\left(I>10^{18} \mathrm{~W} / \mathrm{cm}^{2}\right)$, the quiver energy of an electron oscillating in the electric field of the laser pulse exceeds the electron rest mass, requiring the consideration of relativistic effects. The relativistically 
correct ponderomotive potential is given by

$$
U_{p}=\left(\left[1+\frac{I \lambda^{2}}{1.3 \times 10^{18}}\right]^{1 / 2}-1\right) m_{o} c^{2},
$$

where $I \lambda^{2}$ is the irradiance in $\mathrm{W} \mu \mathrm{m}^{2} / \mathrm{cm}^{2}$ and $m_{o} c^{2}$ is the electron rest mass. At laser irradiance of $I \lambda^{2} \approx 10^{20} \mathrm{~W} \mu \mathrm{m}^{2} / \mathrm{cm}^{2}$, the ponderomotive potential can be of order several MeV. A few recent experiments - discussed in Chapter 3 of this thesis consider this ponderomotive potential sufficiently strong to accelerate protons from the front surface of the target to energies up to tens of $\mathrm{MeV}$.

Another model, known as Target Normal Sheath Acceleration (TNSA), describes the mechanism as an electrostatic sheath on the back surface of the laser target. According to the TNSA model, relativistic hot electrons created at the laser-solid interaction penetrate the foil where a few escape to infinity. The remaining hot electrons are retained by the target potential and establish an electrostatic sheath on the back surface of the target. The electric field associated with this sheath has the form

$$
\vec{E} \approx \frac{k_{B} T_{e}}{e \lambda_{D}}
$$

where $k_{B} T_{e}$ is the electron temperature, $e$ is the electron charge and $\lambda_{D}=\left(\varepsilon_{o} k_{B} T_{e} / e^{2} n_{e}\right)^{1 / 2}$ is the standard Debeye length. Typical electron temperatures and scale lengths of $k_{B} T_{e} \sim 2 \mathrm{MeV}$ and $\lambda_{D} \sim 2 \mu \mathrm{m}$ for ultra-intense laser-plasma interactions can result in an electric field on the back surface of the target of $\vec{E}>10^{12} \mathrm{~V} / \mathrm{m}$. At this field strength, contaminants on the back surface of the target are field ionized and accelerated to high energies over the short scale length of the electric field. Protons 
are preferentially accelerated due to their high charge-to-mass ratio and subsequently shield heavier ions from the electric field.

In this thesis we present several experiments that study the accelerated ions by affecting the contamination layer from which they originate. Radiative heating was employed as a method of removing contamination from palladium targets doped with deuterium. We present evidence that ions heavier than protons can be accelerated if hydrogenous contaminants that cover the laser target can be removed. We show that deuterons can be accelerated from the deuterated-palladium target, which has been radiatively heating to remove contaminants. Impinging a deuteron beam onto a tritiated-titanium catcher could lead to the development of a table-top source of short-pulse, 14-MeV fusion neutrons.

We also show that by using an argon-ion sputter gun, contaminants from one side of the laser target can be selectively removed without affecting the other side. We show that irradiating a thin metallic foil with an ultra-intense laser pulse produces a proton beam with a yield of $1.5-2.510^{11}$ and temperature, $k T=1.5 \mathrm{MeV}$ with a maximum proton energy $>9 \mathrm{MeV}$. Removing contaminants from the front surface of the laser target with an argon-ion sputter gun, had no observable effect on the proton beam. However, removing contaminants from the back surface of the laser target reduced the proton beam by two orders of magnitude to, at most, a yield of $\sim 10^{9}$ and a maximum proton energy $<4 \mathrm{MeV}$. Based on these observations, we conclude that the majority $(>99 \%)$ of high energy protons $(E>5 \mathrm{MeV})$ from the interaction 
of an ultra-intense laser pulse with a thin foil originate on the back surface of the foil - as predicted by the TNSA model. Our experimental results are in agreement with PIC simulations showing back surface protons reach energies up to $13 \mathrm{MeV}$, while front surface protons reach a maximum energy of $4 \mathrm{MeV}$.

Well diagnosed and controllable proton beams will have many applications: neutron radiography, material damage studies, production of medical isotopes, and as a high-resolution radiography tool for diagnosing opaque materials and plasmas. Well collimated and focusable ion beams may also prove beneficial for alternative inertialfusion concepts such as proton fast ignition, a potentially viable method for achieving a controlled fusion reaction in the laboratory earlier than expected.

Edward Morse

Dissertation Committee Chair 


\section{Contents}

List of Figures $\quad$ iii

List of Tables $\quad$ v

1 Introduction $\quad 1$

1.1 The Promise of Fusion Power . . . . . . . . . . . . . . . . . 2

1.2 Basic Plasma Properties . . . . . . . . . . . . . . . . 6

1.3 Inertial Confinement Fusion . . . . . . . . . . . . . . . . . 8

1.4 Possibilities for Fast Ignition . . . . . . . . . . . . . . . . . . . . 12

1.5 Outline of the Thesis . . . . . . . . . . . . . . . . 14

2 Ultra-Intense Laser-Plasma Interactions 17

2.1 High Power Lasers . . . . . . . . . . . . . . . . . . . . . . . . . . . . 18

2.2 Absorption Mechanisms ... . . . . . . . . . . . . . 28

2.2.1 Fundamental Plasma Parameters . . . . . . . . . . . . . . 28

2.2.2 Inverse Bremsstrahlung . . . . . . . . . . . . . . . . . . . 31

2.2.3 Resonant Absorption . . . . . . . . . . . . . . . . . 32

2.2.4 Vacuum Heating . . . . . . . . . . . . . . . . . . . . 34

2.3 The Ponderomotive Force . . . . . . . . . . . . . . . . . 34

2.3.1 Overview and Historical Background . . . . . . . . . . . . . . 34

2.3.2 Single-Particle Derivation of the Ponderomotive Force . . . . . 36

2.3.3 Examples of the Nonlinear Force . . . . . . . . . . . . . . 38

2.3.4 $\vec{J} \times \vec{B}$ Heating . . . . . . . . . . . . . . . . 41

3 Ion Acceleration 43

3.1 Early Ion Acceleration Research . . . . . . . . . . . . . . . . 43

3.1.1 The Los Alamos Experiments . . . . . . . . . . . . . . . . . 47

3.2 The Ponderomotive Potential Revisited . . . . . . . . . . . . . . . 49

3.3 Observation of High Energy Protons . . . . . . . . . . . . . . . . . 51

3.3.1 The Michigan Experiments . . . . . . . . . . . . . . . 52 
3.3.2 The RAL Experiments . . . . . . . . . . . . . . 54

3.3.3 The Petawatt Experiments . . . . . . . . . . . . 56

3.4 Target Normal Sheath Acceleration . . . . . . . . . . . . 58

4 Particle Detection: Instruments and Diagnostics $\quad 62$

4.1 Radiochromic Film . . . . . . . . . . . . . . . . . . 63

4.2 Nuclear Activation Counting . . . . . . . . . . . . . . . . 70

4.2.1 Charged Particle Detection with Titanium . . . . . . . . . 70

4.2.2 Neutron Detection . . . . . . . . . . . . . . . . . . . 72

5 The DT Experiment $\quad 77$

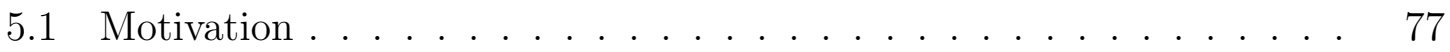

5.2 A Tritiated Titanium Target . . . . . . . . . . . . . . . . . 79

5.3 Production of a Deuteron Beam . . . . . . . . . . . . . . 81

5.4 Neutron Detection . . . . . . . . . . . . . . . . . 88

6 Removal of Surface Contaminants by Ion Sputtering 94

6.1 The Ion Sputter Gun . . . . . . . . . . . . . . . . . . . . . . 95

6.2 Surface Cleaning Experiment . . . . . . . . . . . . . . . . . . . . . . 99

6.3 Adsorption of Contaminants

on Target Surfaces . . . . . . . . . . . . . . . . . . . . . . . 108

6.4 Simulation of Experimental Results . . . . . . . . . . . . . . 111

7 Concluding Remarks and Recommendations for Future Work 118

7.1 Deuteron Acceleration and the Production of DT-Fusion Neutrons . . 119

7.2 The Origin of Accelerated Protons . . . . . . . . . . . . . . 121

7.3 Future Work . . . . . . . . . . . . . . . . . . . . . . 121

A Adsorption of Water onto $\mathrm{Au}$ and $\mathrm{Al}$ Foils $\quad 126$

B Code: Analysis of RCF 135

B.1 Mathematica Script . . . . . . . . . . . . . . . . 136

B.2 Java Script . . . . . . . . . . . . . . . . . . . . . . . . . 145

$\begin{array}{lr}\text { Bibliography } & 157\end{array}$ 


\section{List of Figures}

1.1 A simple power plant energy cycle . . . . . . . . . . . . 5

1.2 ICF capsule composition. . . . . . . . . . . . . . . . . . . 9

1.3 Two main approaches to ICF $\ldots \ldots \ldots \ldots$

1.4 Conventional and Fast Ignition ICF $\ldots \ldots \ldots \ldots$

1.5 Indirect drive with laser accelerated ion beam . . . . . . . . . . 13

2.1 Diagram of stretcher-compressor system for CPA . . . . . . . . . 22

2.2 Diagram of JanUSP laser system . . . . . . . . . . . . . . 26

2.3 P-polarized light at oblique incidence . . . . . . . . . . . . . 33

2.4 The nonlinear force in a plasma . . . . . . . . . . . . 40

3.1 Forces at the critical surface . . . . . . . . . . . . . 50

3.2 Wedge target produces two proton beams . . . . . . . . . . 58

3.3 Schematic of three main processes governing ion acceleration . . . . . 61

4.1 Experimental setup for laser-accelerated ions . . . . . . . . . . 65

4.2 Deposited dose in krad as a function of optical density for RCF . . . 66

4.3 RCF (MD-55) from JanUSP shot\# 10011605. . . . . . . . . . . 67

4.4 Analysis of JanUSP shot\# 10011605. . . . . . . . . . . . 68

4.5 Maxwellian dist. of proton beam. . . . . . . . . . . . . . 69

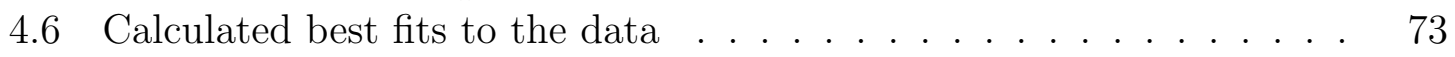

4.7 The DT-fusion cross section . . . . . . . . . . . . . . . . 74

4.8 Gold activation cross sections and activation spectrum $\ldots \ldots \ldots$

5.1 Schematic of DT experiment. . . . . . . . . . . . 78

5.2 Diagram of stainless-steel assembly that contains the tritiated-titanium disk . . . . . . . . . . . . . . . . . . 80

5.3 Schematic of heavy-water electrolytic cell. . . . . . . . . . 83

5.4 SIMS analysis of deuterated palladium foil . . . . . . . . . . 84

5.5 Radiative heater . . . . . . . . . . . . . . . . . . . . 85

5.6 Shot series on $25-\mu \mathrm{m}$ thick Pdfoils . . . . . . . . . . 86 
5.7 Quantitative analysis of proton beam from Pd targets . . . . . . . . 87

5.8 Gold activation data. . . . . . . . . . . . . . . . . . . . . . . . . 89

6.1 Kaufman type, permanent magnet $3 \mathrm{~cm}$ Ion Source . . . . . . . . . 95

6.2 Ion sputter gun shown in the JanUSP target chamber. . . . . . . . . 98

6.3 Experimental setup of ion sputter gun cleaning experiment. . . . . . . 100

6.4 Observed proton beam from $15-\mu \mathrm{m}$ thick $\mathrm{Al}$ targets. . . . . . . . . 102

6.5 XPS analysis of $\mathrm{Al}$ and $\mathrm{Au}$ targets. . . . . . . . . . . . . . . 103

6.6 Observed proton beam from $15-\mu \mathrm{m}$ thick Au targets. . . . . . . . . 106

6.7 Proton spectra from $15-\mu \mathrm{m}$ thick $\mathrm{Au}$ targets fit to a Maxwellian distribution. . . . . . . . . . . . . . . . 107

6.8 Composition and relative pressures of residual gas in JanUSP target chamber. . . . . . . . . . . . . . . . . . . . . . . 109

6.9 Lewis dot model of $\mathrm{H}_{2} \mathrm{O}$ molecule. . . . . . . . . . . . . . . . 110

6.10 Particle-in-cell simulation density profile . . . . . . . . . . . . 114

6.11 Particle-in-cell simulation density profile . . . . . . . . . . . 115

7.1 Flat foil and focusing target experiment. . . . . . . . . . . . . 123

7.2 Typical ICF targets with reentry cones . . . . . . . . . . . . . 124

A.1 Qualitative drawing of adsorption potential energy curve. . . . . . 128

A.2 3-D plot of the adsorbate concentration of water vapor on gold. . . . 132

A.3 3-D plot of the adsorbate concentration of water vapor on aluminumoxide. . . . . . . . . . . . . . . . . . 133 


\section{List of Tables}

1.1 World Consumption of Primary Energy . . . . . . . . . . . . . . 3

1.2 Comparison of isobaric and isochoric . . . . . . . . . . . 12

2.1 Laser gain media characteristics . . . . . . . . . . . . . . . 20

2.2 LLNL JanUSP and Petawatt laser system characteristics. . . . . . . . 27

4.1 Specifics of GAFCHROMIC Radiochromic film . . . . . . . . . . . 64

6.1 IBS 250 Power Supply. . . . . . . . . . . . . . . . . . . . . . . . . . . . . . 97

6.2 FIBS potentials . . . . . . . . . . . . . . . . 113 


\section{Acknowledgments}

Due to the fact that my doctoral studies were spent between the Nuclear Engineering Dept. at UC Berkeley and Lawrence Livermore National Laboratory, I have many people to acknowledge.

First and foremost I must thank my advisor, Prof. Edward Morse. His unfaltering support, during times of academic, emotional, and occasionally financial turmoil, made it possible to remain in graduate school without losing my sanity. I have the greatest admiration for his command of plasma physics and the nuclear sciences on both an experimental and theoretical level and hope to one day have at least a comparable amount of knowledge myself.

I would also like to thank Tom Cowan, my first advisor on the LLNL side for accepting me as a student of laser-nuclear physics and for being very understanding when personal matters required me to take a leave of absence from the lab.

I would like to thank Pravesh Patel my ultimate advisor on the LLNL side for accepting me as his very first student when Tom Cowan, among others, left the lab to pursue other opportunities. Dr. Patel's acumen of physics was as great as his patience in matters of the lab and the governing bureaucracy. His guidance and reassurance in times of uncertainty and anxiety were both a comfort and a blessing.

Dwight Price, V-division's principle laser scientist, was indispensable during our experimental time on the JanUSP laser. His willingness to stay late at the lab and tackle laser system malfunctions as they arose, enabled us to get the quality data 
that is presented later in this thesis.

Our experiments involving radioactive materials could not have been performed without the help of Sean Holte, V-division's safety officer. Sean's understanding of how research is preformed in the lab allowed us to establish operating procedures that were practical as well as compliant with safety regulations.

I would like to thank the staff of the Student Employee Graduate Research Fellowship (SEGRF) program for funding my doctoral studies and quickly resolving bureaucratic difficulties that inevitably all students at LLNL encounter. The decision to consolidate the SEGRF and other student programs under one office of student affairs was one of the best administrative decisions I witnessed during my time at the lab.

Thanks also go to Ken Wharton, for many enjoyable lunchtime conversations at the lab, and for letting me use the style of his Ph. D. thesis ${ }^{1}$ as model for my own. I look forward to the day when all (or at least a few) of Ken's "whacky theories" are shown to be true, and I can say I was in the presence of greatness.

At UC Berkeley, I have to thank Lisa Zemelman, our department's graduate student assistant, for helping to resolve various problems that arose and for being understanding when it took me so long to register for classes. Thanks also go to my office mates, Carlos Barrera and Dan Hua, for ensuring I had at least two-square feet of usable desk space.

\footnotetext{
${ }^{1}$ Laser Plasma Interactions Relevant to Inertial Confinement Fusion, K. B. Wharton, UCLA (1998), LLNL UCRL-LR-132566.
} 
Most heartfelt thanks go to Poul Petersen one of my fellow My-House residents and dear friend. As well as being able to answer questions in almost any branch of science, he laughed at my quirky physics humor and instilled in me a vast knowledge of the great state of Denmark that will surely be of immeasurable value when I'm Secretary øf State.

Of course all of my housemates and friends have been a great source of joy and inspiration, throughout my time Berkeley. Thanks to Leah Dorazio (one of my first friends at Berkeley) for being there during good and bad times, and for helping me debug the Java code used in my research. Thanks to Tina Green for suffering through my questions on economics and getting me a discount on my subscription to Foreign Affairs. Thanks to Axel Seemann for raising the academic level of our Sunday Supper conversations and for introducing us to terrible towny bars and drink concoctions whose originality was rivaled only by their ghastly taste. Thanks to these and the many others Helen, Ula, G.L., Boris, Jen, Derek and Sarah for putting up with me and not having me committed when obsession drove me to make the argument: Why I should be on Roswell.

Finally, I would like to thank my mom and sister for their kind and loving support throughout the many years I spent in graduate school when I could have been making money to pay back college loans. 


\section{Chapter 1}

\section{Introduction}

This chapter introduces the benefits of fusion power as a means of producing commercially available electricity. Basic plasma physics parameters relevant to the study of fusion plasmas are defined, and multiple approaches to achieving fusion power are briefly discussed. Inertial Confinement Fusion (ICF), which uses lasers or heavy ion beams to compress a small pellet filled with a fuel of deuterium and tritium, is shown as a promising candidate for fusion power. The fast ignitor concept, a potentially easier but as-of-yet-unvalidated approach to ICF, is described along with recent ion-acceleration experiments that could increase the concept's feasibility. An outline of this thesis describing laser-plasma, ion-acceleration experiments is given in Section 1.5. 


\subsection{The Promise of Fusion Power}

The availability of power is a primary interest to every nation or political state. whether power is consumed in the form of electricity or as horsepower in vehicles, it is essential for security and economic well-being. The availability of power is dependent on two things: a source of energy and a means to convert that energy into a useful form of power such as electricity.

Sources of energy can be classified as either renewable or exhaustible. The later form such as coal, oil, and natural gas represent $>80 \%$ of the world (primary) energy use. Renewable sources such as solar radiation, wind, and hydro account for $<10 \%$ of the world energy use. Nuclear fission as an energy source is difficult to classify as either renewable or exhaustible since reactors could be constructed to use nuclear fuel in an inexhaustible manner, but political forces (not technological barriers) have so far prevented such construction. Fission is also difficult to classify environmentally since, although fission power plants do not produce the greenhouse gases that contribute to global warming, they do produce measurable amounts of radioactive waste, which some consider intolerable.

Nations and states have generally chosen to use exhaustible sources of energy, which contribute to pollution and rising global temperatures, rather than renewable forms of energy, which are less harmful to the environment, because the former is more efficiently converted to power. Fossil fuels and nuclear energy can be transported to almost any location, unlike most forms of renewable energy which require the sun 


\begin{tabular}{lcc}
\hline \hline Source & Quadrillion $\left(10^{15}\right)$ Btu & Percent of Total \\
& & \\
& & \\
Petroleum & 156.48 & 38.7 \\
Natural Gas & 93.11 & 23.1 \\
Coal & 95.94 & 23.8 \\
Nuclear & 26.45 & 6.5 \\
Hydro & 26.85 & 6.6 \\
Geothermal, & 3.11 & 0.8 \\
Solar, Wind & & \\
\hline \hline
\end{tabular}

Table 1.1: World Consumption of Primary Energy, 2001. Data taken from Department of Energy, Energy Information Administration. www.eia.doe.gov

to shine, the wind to blow, or a river to flow somewhat near those requiring power. Nuclear fusion, the process that powers the Sun, will be superior to all forms of power production currently in use by providing an inexhaustible source of energy that does not contribute to global warming and is low in radioactive waste.

Fusion is the combination of light nuclei into a heavier nucleus. Fusion reactions are exothermic, with the energy produced equal to the mass difference between the sum of the products and the sum of the reactants, according to Einstein's famous relativity equation $E=\Delta m c^{2}$. Common fusion reactions involving isotopes of hydrogen such as deuterium (D) and tritium (T) and the energy associated with each product are: 


$$
\begin{array}{ll}
\mathrm{D}+\mathrm{D} & \longrightarrow \mathrm{T}(1.01 \mathrm{MeV})+p(3.02 \mathrm{MeV}) \\
\mathrm{D}+\mathrm{D} & \longrightarrow{ }^{3} \mathrm{He}(0.82 \mathrm{MeV})+n(2.45 \mathrm{MeV}) \\
\mathrm{D}+\mathrm{T} & \longrightarrow{ }^{4} \mathrm{He}(3.5 \mathrm{MeV})+n(14.1 \mathrm{MeV}) \\
\mathrm{D}+{ }^{3} \mathrm{He} & \longrightarrow{ }^{4} \mathrm{He}(3.6 \mathrm{MeV})+p(14.7 \mathrm{MeV}) \\
\mathrm{T}+\mathrm{T} & \longrightarrow{ }^{4} \mathrm{He}+2 n+11.3 \mathrm{MeV}
\end{array}
$$

We can see the energy released in the above nuclear reactions is in the $\mathrm{MeV}$ range. This is orders of magnitude higher than the energy released in the breaking of chemical bonds associated with the burning of fossil fuel. Fusion fuel is also more plentiful since deuterium, a naturally occurring isotope of hydrogen, is found in water at a concentration of approximately 1 in 7,000 parts. The Earth's oceans, therefore, provide an essentially infinite source of fusion energy if it can be efficiently converted to a useful form of power.

The production of electricity - arguably the most useful form of power - has been the main goal of fusion research for the past 50 years. If the energy released in fusion reactions can be produced efficiently and used as a heat source for steamturbine generators, the production of greenhouse gases could be greatly reduced and perhaps relegated only to the transportation sector. The fact that several fusion reactions produce charged particles has lead some to consider the possibility of producing electricity directly, without the need of steam-turbine generators, which have a low efficiency of $\sim 40 \%$. Unfortunately, releasing the energy stored in the nuclei of atoms 


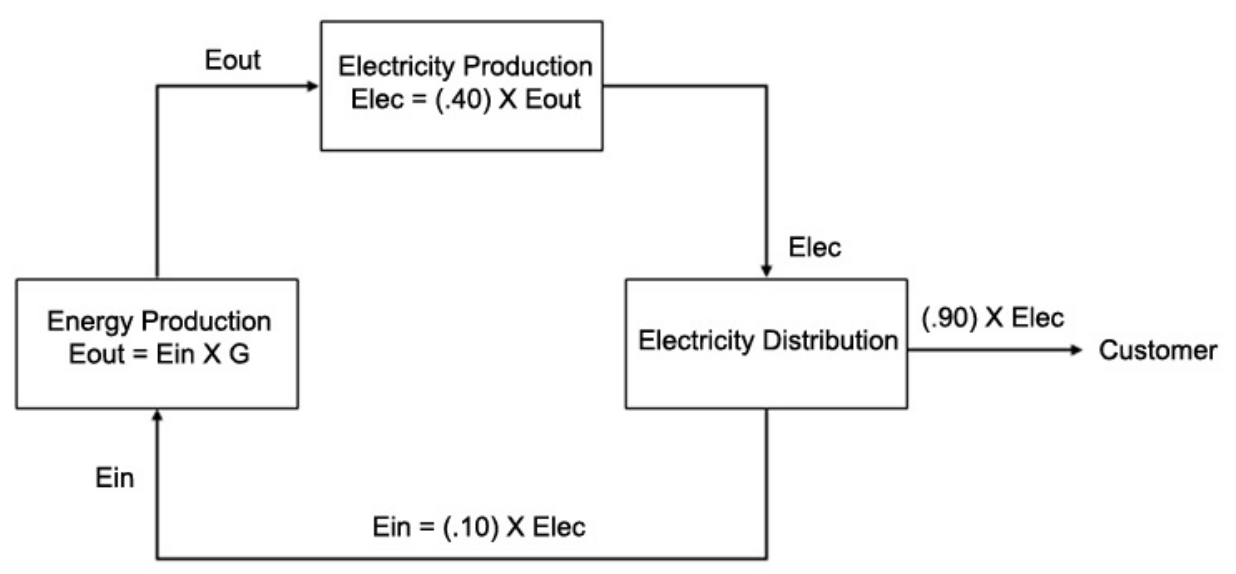

Figure 1.1: A simple power plant energy cycle including only an inefficiency of $40 \%$ in conversion to electricity and $10 \%$ return to plant predicts a necessary gain of $G=25$.

is more difficult than releasing the energy stored in chemical bonds of fossil fuels.

The usefulness of an energy source is often described in terms of gain $(G)$, the amount of energy produced divided by the amount of energy consumed. Referring to the simple energy cycle shown in Fig. 1.1, we can derive the required gain for a power plant producing commercial electricity. As stated above, the amount of energy produced $E_{\text {out }}$ is converted into electricity with an efficiency of $40 \%$. After its generation, the electricity is distributed with $90 \%$ going to the customer and $10 \%$ being used to run the plant. We can calculate the gain required to run such a plant by solving for $G$,

$$
G=\frac{E_{\text {out }}}{E_{\text {in }}}=\frac{E_{\text {out }}}{.10 \times \text { Elec }}=\frac{E_{\text {out }}}{.10 \times .40 \times E_{\text {out }}}=\frac{1}{.10 \times .40}=25 .
$$

This simple calculation shows that a gain of 25 is required to run the power plant, and we have not even accounted for inefficiencies in energy production. If we include 
the inefficiencies associated with energy production, we would quickly reach a gain of $G>100$. So far, the only man-made fusion devices to achieve a gain greater than one have been hydrogen bombs and associated nuclear weapons $(G>>1000)$; not a safe choice for the production of commercial electricity.

Before fusion energy can be considered a replacement for fossil fuel, a practical level of gain must be achieved in the laboratory in a containable and reliable manner. Laser fusion, a possible method of achieving break even $(G=1)$ and controllable high gain $(G>100)$, is presented in the next several sections and throughout the remainder of this thesis.

\subsection{Basic Plasma Properties}

In order for nuclei to fuse, they must get very close to each other; that is, they must have sufficient energy to overcome the Coulomb barrier associated with the nucleus. At these energies (several keV or higher), light elements would be partially or completely ionized, existing as dissociated positively charged ions and negatively charged electrons. This state of existence is considered the fourth state of matter the first three states being solid, liquid, and gas - or the plasma state:

We can define a plasma as a quasi-neutral gas of charged and uncharged particles that exhibit a collective behavior. [1]

Since plasmas require high temperatures to sustain ionization, most naturally occurring plasmas can only be found in exotic locations such as stellar interiors and 
atmospheres. Although temperatures on the Earth are often too low to sustain plasmas for any substantial amount of time, a few terrestial examples do exists such as the flash of a lightning bolt, the Northern and Southern Lights, and the conducting gas inside a fluorescent tube or neon sign.

Two dominant characteristics of a plasma are its temperature and its density or number of particles. As stated above the temperature or kinetic energy of the plasma must be higher than the potential energy of the charged particles. At such energies, plasmas have the ability to distribute themselves in order to shield out electric potentials that are applied to it. This means that negative charges will gather around positive charges effectively shielding positive charges from feeling the effects of one another. It is this process of charge shielding that gives the plasma its quasineutral property. The characteristic length over which shielding occurs is given by the Debye length,

$$
\lambda_{D}^{2} \equiv\left(\frac{\epsilon_{o} k_{B} T_{e}}{n e^{2}}\right),
$$

where $k_{B}$ is the Boltzmann constant, $T_{e}$ is the electron temperature, $n$ is the electron density, and $e$ is the electron charge.

Clearly, the definition for Debye length is only valid if there are enough particles in the plasma to constitute an effective shield. We can compute the number, $N_{D}$, of particles in a "Debye sphere" by presuming $\lambda_{D}$ is the sphere's radius:

$$
N_{D}=n \frac{4}{3} \pi \lambda_{D}^{3} .
$$


For a plasma to exhibit "collective behavior", the number of particles in the Debye sphere must be much greater than one: $N_{D} \gg 1$.

\subsection{Inertial Confinement Fusion}

For a plasma of hydrogen isotopes to produce a sustained fusion reaction, the ions must be confined long enough to contact one another. In 1972, Knuckles et al. proposed the use of laser beams to heat and compress a spherical capsule with a shell composed of an outer ablator region, and an inner region of cryogenic DT fuel [2]. Typical capsule composition - as thought to be required for ignition - is depicted as a wedge in Fig. 1.2. Energy from the laser driver is delivered to the ablator, which heats up and expands. To conserve momentum, this expansion produces a rocket-like acceleration of the fuel inward. In the final stage of compression, the fuel consists of two distinct regions - a central "hot spot," containing $\sim 2-5 \%$ of the main fuel and a dense, cold main fuel region comprising the remaining mass [3]. If the areal density of the hot spot $\left(\rho R_{\text {hot }}\right)$ is of order $0.3 \mathrm{~g} / \mathrm{cm}^{2}$, which, in a $5-10 \mathrm{keV}$ plasma is approximately the range of the $3.6 \mathrm{MeV}$ alpha particle produced by the DTfusion reaction, then the alpha particles will stop within the hot spot further raising the plasma temperature, causing a propagating burn wave through the remaining compressed cold fuel [4].

Compressing the target to high density will produce an ion-ion collision time that is much shorter than the disassembly time of the exploding pellet. This scheme, 


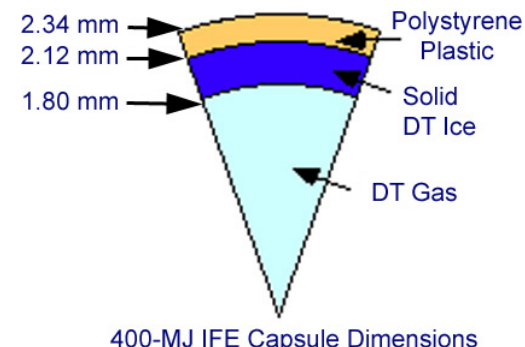

400-MJ IFE Capsule Dimensions
Figure 1.2: Composition of ICF capsule thought to be required for ignition. Spherical capsules are filled with an equal mixture of deuterium and tritium, and then chilled to cryogenic temperatures
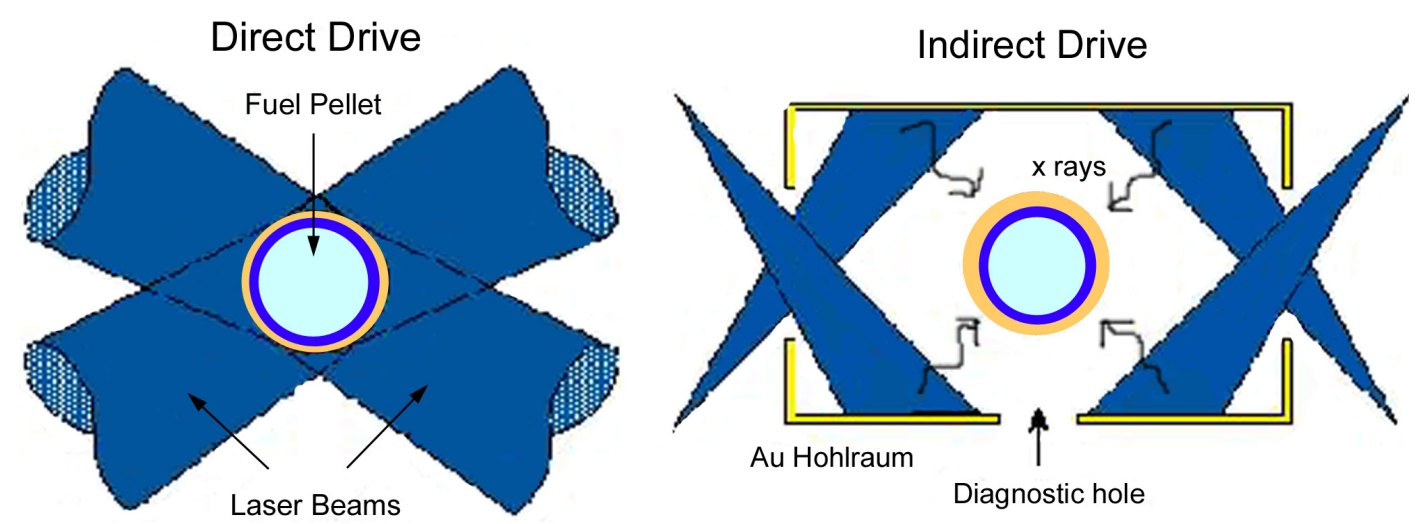

Figure 1.3: Two main approaches to ICF are Direct Drive in which laser beams are focused directly onto the fuel pellet and Indirect Drive in which the lasers interact with the inner walls of the hohlraum.

known as inertial confinement fusion (ICF) has many variations, all of which rely on the fundamental requirement that the inward inertia of the pellet compress the fuel; the fuel burns, releasing more energy than was used to compress it, before the target disassembles.

There exist two main experimental and theoretical approaches to ICF; both are depicted in Fig. 1.3. In the first, known as Direct Drive, laser beams are used to uniformly irradiate a spherical DT fuel pellet. The laser energy is transferred to electrons and ions by processes such as inverse bremsstrahlung and various plasma 


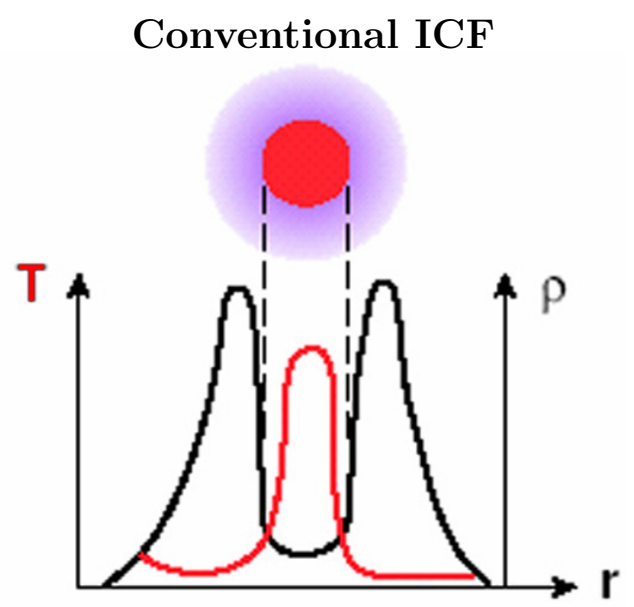

(a) $\rho k_{B} T_{\text {hot }} \approx \rho k_{B} T_{\text {cold }}$ (isobaric)
Fast Ignitor

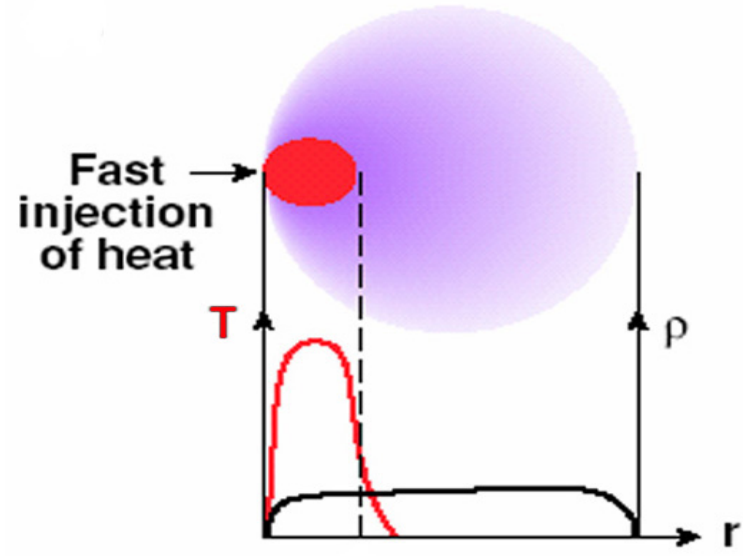

(b) $\rho_{\text {hot }} \approx \rho_{\text {cold }}$ (isochoric)

Figure 1.4: (a) Low-density central spot ignites a high-density cold shell. (b) Rapidly heated side spot ignites high-density fuel.

absorption mechanisms discussed in the next chapter.

Indirect Drive is similar to Direct Drive, but constraints on the latter by the need for high quality irradiation uniformity and compression symmetry are reduced by indirectly heating the fuel pellet with secondary $\mathrm{x}$ rays. In the Indirect Drive approach, the fuel capsule is mounted inside of a high-Z metallic container called a hohlraum $^{1}$. Hohlraums can be cylindrical with 2 Laser Entrance Holes (LEHs) or tetrahedral with 4 LEHs and are typically made of gold because of gold's high laserto-x ray conversion efficiency. The driver energy is absorbed in the hohlraum walls that surround the capsule. The heated wall material emits $\mathrm{x}$ rays, which drive the capsule implosion [3].

Both of the above techniques require an "isobaric compression" of the fuel pellet,

\footnotetext{
${ }^{1}$ The word hohlraum is simply the German word for a hollow space.
} 
so called because the two regions of the hot and cold fuel are said to be in pressure equilibrium [5]. An alternative model known as the "fast ignitor" was proposed by Tabak et al. [6], in which the fuel is compressed by conventional driver methods and a separate ultra-intense laser or particle pulse is used to deliver energy sufficiently rapidly to ignite the fuel at a much lower peak density. A schematic of how the two approaches differ is presented in Fig. 1.3. A lower fuel density allows a more favorable gain calculation based on the isochoric (uniform density) model of Kidder [7]. A quantitative comparison of the two models is presented in Table 1.2.

A lower peak density reduces the necessary driver energy to compress the target, analogous to our $E_{i n}$ in Fig. 1.1. More fuel mass available for burn means more energy produced, analogous to a larger $E_{\text {out }}$. Increasing the energy produced, while decreasing the required input energy results in a larger gain. As we can see from Table 1.2, the gain predicted by the isochoric model is $\sim 20$ times higher than that of the isobaric model.

The main obstacle in the fast-ignitor approach is finding the means of depositing enough energy to ignite a hot spot in the pellet before pressure equilibrium can reestablish itself. The advent of short-pulse, ultra-intense lasers may provide the necessary means of overcoming this obstacle. 


\begin{tabular}{lcc}
\hline \hline Property & Isobaric model & Isochoric model \\
& & \\
\hline & 380 & 7300 \\
Gain & 220 & 880 \\
$R_{\text {main fuel }}(\mu \mathrm{m})$ & 420 & 42 \\
$\rho_{\text {main fuel }}\left(\mathrm{g} / \mathrm{cm}^{3}\right)$ & 12 & 120 \\
Fuel mass $(\mathrm{mg})$ & & \\
\end{tabular}

Table 1.2: Comparison of optimal quantities in the isobaric and isochoric models. Based on a coupling efficiency of $30 \%$ and incident driver energy of $2 \mathrm{MJ}$ as predicted for the NIF. Adapted from Tabak et al. [6].

\subsection{Possibilities for Fast Ignition}

Fast ignition requires the precompression of a fuel pellet and the subsequent injection of a short-pulse, ultra-intense laser or particle (electron or ion) pulse. Several technical questions remain to be answered as to which type of pulse will have the highest probability of success. Although short-pulse laser technology has advanced very rapidly in the last decade and has shown that lasers can produce intense beams of relativistic electrons, it is not clear that they can produce a well collimated electron beam capable of depositing sufficient energy into the compressed fuel. Heavy-ion beams have been considered a viable candidate for fast ignition given their high coupling efficiency to the fuel at the end of their range (Bragg peak). However, delivering heavy-ion beams in the small spot sizes and short pulse lengths necessary for fast ignition (as well as heavy-ion driven ICF in general) is still a large technical difficulty the heavy-ion research community is working to overcome. 


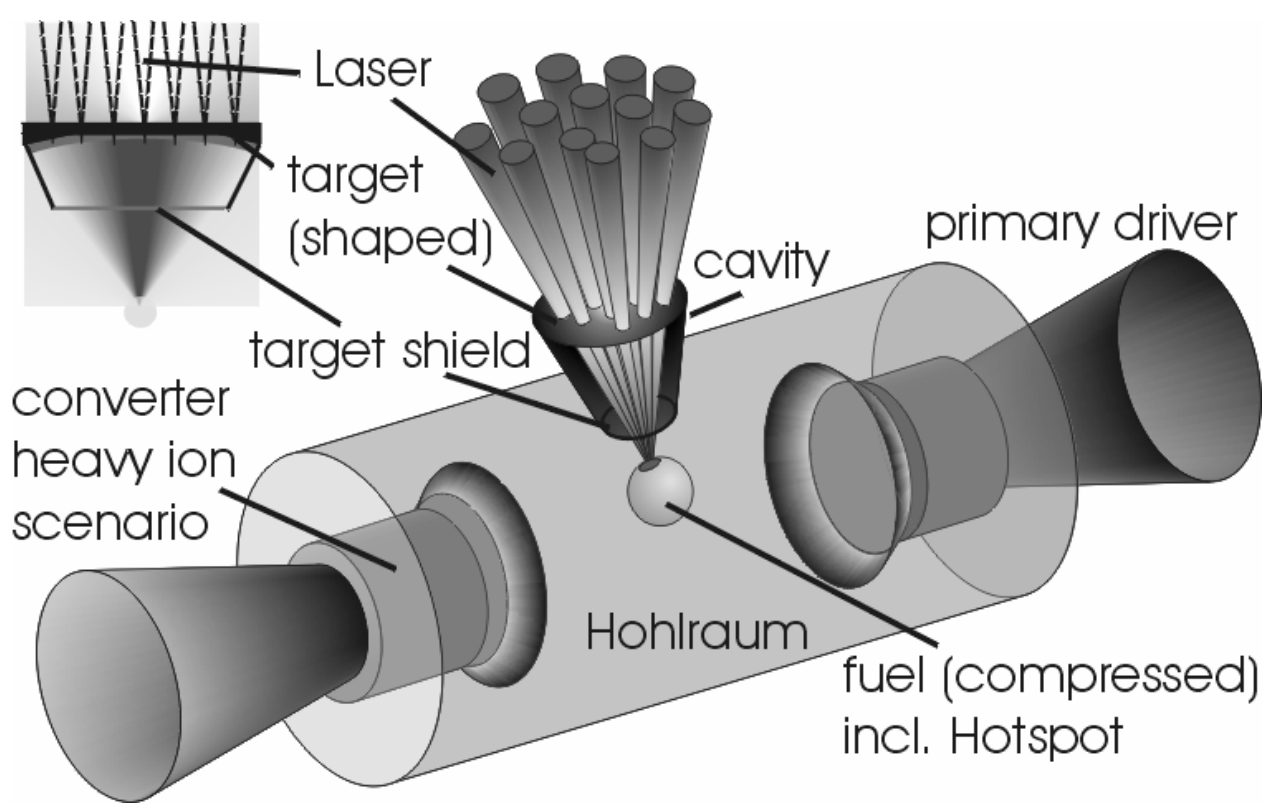

Figure 1.5: Indirectly driven fast ignition using a laser accelerated proton beam. The rear surface of the laser-target is shaped to focus the proton beam into a suitable spot size for ignition. Used with permission from M. Roth [8].

The discovery that ultra-intense laser could generate high yields of well collimated protons, as described by Roth et al., immediately made possible a means of iondriven fast ignition, "while circumventing the difficulties of ion acceleration, pulse compression, focusing and transport [8]." The concept of using laser-accelerated proton beams for fast ignition is shown in Fig. 1.5. Multiple petawatt-class lasers are focused onto a target in close proximity to the compressed fuel pellet. An intense beam of protons is accelerated from the rear surface of the target, which is curved to ballistically focus the beam to the desired spot size. For specificity, Fig. 1.5 shows a hohlraum heated by heavy-ion beams, but the target design could easily be adapted to laser-driven Indirect Drive or even Direct Drive scenarios. 


\subsection{Outline of the Thesis}

Before laser-accelerated ion beams can be tailored to the conditions required for fast ignition, the governing physics of the phenomenon must be understood. To provide further understanding of laser-accelerated ion beams is the goal of this thesis.

Chapter 2 provides a brief history of high power lasers and the relevant concepts of laser-plasma interactions to ion acceleration. Chirped pulse amplification of laser pulses to the ultra-intense regime is described, along with two high-power laser systems that have been used in ion-acceleration research: the Petawatt laser and the JanUSP laser both at Lawrence Livermore National Laboratory. Several energy absorption mechanisms that transfer energy from the laser pulse to the plasma are presented. The nonlinear ponderomotive force is discussed along with examples such as self-focusing and $\vec{J} \times \vec{B}$ heating.

Chapter 3 provides a review of the physical mechanisms presently used to describe ion acceleration from laser-plasma interactions. The chapter begins with the early Helios experiments at Los Alamos National Laboratory, in which hydrogenous contaminants on laser targets were accelerated to energies of tens of $\mathrm{keV}$. Then, the ponderomotive force is re-presented in the modern ultra-intense regime as a means of calculating the velocity attained by ions as a result of high radiation pressure at the laser-plasma interface. Recent experiments that have observed ions accelerated to energies in the $\mathrm{MeV}$ range are presented. A remaining question in the field as to the dominant acceleration mechanism - front-surface ponderomotive acceleration, back- 
surface electrostatic sheath acceleration, or a combination of the two - is presented in this chapter and discussed throughout the remainder of this thesis.

Chapter 4 describes the methods and diagnostics of particle detection used in ion-acceleration experiments presented in this thesis. The two predominant means of particle detection used in the experiments described here were radiochromic film, a film sensitive to radiation dose, for the detection of protons and nuclear activation of gold foils for the detection of neutrons.

Chapter 5 presents results from an experiment attempting to show that removing hydrogenous contaminants by radiative heating allows for the acceleration of heavier ions. Deuterons - which have a charge-to-mass ratio one half that of protons - were accelerated by radiatively heating deuterated targets prior to laser irradiation. The accelerated deuterons propagated toward a tritium target and neutrons from the DT-fusion reaction were detected by nuclear activation of gold foils. Fabrication of deuterated-palladium targets is discussed, as well as the construction of a novel sealed tritium target that could be used in the laser-target chamber with little or no tritium contamination.

Chapter 6 presents results from a second approach at removing hydrogenous contaminants by using an argon ion-sputter gun to selectively etch one side of the lasertarget. Aluminum and gold targets of $15-\mu \mathrm{m}$ thickness were "cleaned" on one side at a time (either front surface or back surface), and the resulting proton beam was recorded on radiochromic film. 
The final chapter, Chapter 7, concludes this thesis with a discussion of the implications for ion acceleration with respect to the fast ignitor concept, remaining questions and proposals for future work. The effectiveness of removing hydrogenous contaminants by radiative heating and ion-sputter cleaning is discussed, and contributions this thesis made to further our understanding of the physical processes related to the acceleration of ions in laser-plasma interactions are summarized. 


\section{Chapter 2}

\section{Ultra-Intense Laser-Plasma}

\section{Interactions}

As high power lasers are required for all conceivable forms of laser-fusion power, we begin this chapter with a brief history of high power laser technology. We then describe fundamental parameters associated with laser-plasma interactions and dominant absorption mechanisms that govern the transfer of energy from a laser pulse to a plasma. We conclude with examples of why the ponderomotive force and other nonlinear forces are highly relevant in the regime of ultra-intense, short-pulse laserplasma interactions. 


\subsection{High Power Lasers}

Since the first demonstration of the optical laser in 1960, advances in peak laser power have moved in leaps and bounds $[9,10]$. The very first lasers were continuous wave $(\mathrm{CW})$ or free running lasers with powers of kilowatts. The discovery of Qswitching quickly after the laser's first demonstration raised the peak power of pulsed systems to that of megawatts. Mode locking in the 1970s raised the peak power by another three orders of magnitude up to the gigawatt level. Peak laser power reached a plateau at this level and remained there for almost a decade. Then, two very important discoveries in the mid-1980's, the ability to generate ultra short pulses and the application of Chirped Pulse Amplification (CPA) to solid state lasers [11], paved the way for petawatt laser pulses, currently the highest producable laser power.

The two most direct methods of increasing laser peak power are increasing the energy of the laser pulse and/or decreasing the pulse duration. Until the early 1990s, high power was mainly achieved by adding amplifiers in the laser chain, thereby increasing the energy of the shortest possible pulses (nanosecond pulse duration) that could be reliably generated at the time. In 1991, the discovery of Kerr-lens mode locking [12] made it possible to routinely and reliably generate pulses as short as $10 \mathrm{fs.}$ When mode locking conditions are met, the electric field amplitude of the laser pulse is given by the Fourier transform of the magnitude of the spectral amplitude, and the pulse width, $\Delta \tau$, is related to the width of the laser pulse spectrum, $\Delta \nu$, by the 
relation,

$$
\Delta \tau \Delta \nu=\beta,
$$

where $\beta$ is a numerical factor of order unity [13]. The exact value of $\beta$ depends on the particular shape of the spectral intensity distribution. For a Gaussian pulse, $\beta=2 \ln 2 / \pi$, which limits the bandwidth product to $\Delta \tau \Delta \nu \geq 0.441$. Pulses of this sort are said to be transform limited.

Armed with femtosecond pulses, the only thing laser scientists needed to reach the highest attainable peak powers was a method of amplification.

The first requirement of any amplifying medium for short pulse lasers, is that the bandwidth of the amplifier be large enough to accommodate the full spectrum of the short pulse. Table 2.1 lists several popular gain media that are currently used such as neodymium-doped glass (Nd:glass) and titanium-doped sapphire (Ti:sapp) along with several that are still being developed (Alexandrite). Ti:sapphire, a material developed at the Lincoln Laboratory of the Massachusetts Institute of Technology [14, 15], is currently popular as a gain medium since it can theoretically support the shortest possible pulses approaching $\Delta \tau<5$ fs.

The second criterion to be met in the amplifying medium is the ability to efficiently extract energy stored in the amplifier. In order to accomplished this, the fluence of the pulse must to be near the saturation fluence of the medium defined as,

$$
F_{s a t}=\frac{h \nu}{\sigma},
$$

where $h$ is Planck's constant, $\nu$ is the laser frequency, and $\sigma$ is the medium's stimulated 


\begin{tabular}{ccccc}
\hline \hline Amplifying Medium & $\sigma$ & $\Delta \lambda$ & $\Delta \tau$ & $P_{t h}$ \\
& $\left(10^{-20} \mathrm{~cm}^{2}\right)$ & $\begin{array}{c}\Delta \mathrm{nm}) \\
(\mathrm{fs})\end{array}$ & $\left(\mathrm{TW} / \mathrm{cm}^{2}\right)$ \\
& & & & \\
Nd:glass phosphate & 4 & 22 & 80 & 60 \\
Nd:glass silicate & 2.3 & 28 & 60 & 100 \\
Nd:glass combination & 1.5 & 60 & 30 & 400 \\
Ti:sapphire & 30 & 120 & 8 & 120 \\
Alexandrite & 1 & 100 & 10 & 2000 \\
Cr:LiSAF & 3 & 50 & 15 & 300 \\
& & & & \\
\hline \hline
\end{tabular}

Table 2.1: laser gain media characteristics

emission cross section. Most materials used as gain media for solid state lasers have a saturation fluence between $1 \mathrm{~J} / \mathrm{cm}^{2}$ (Ti:sapphire) and $6 \mathrm{~J} / \mathrm{cm}^{2}$ (Nd:glass). The theoretical peak power $P_{t h}$ can be estimated as the saturation fluence divided by the pulse duration,

$$
P_{t h}=\frac{F_{s a t}}{\tau}=\frac{h \nu}{\sigma \tau} \approx 2 \frac{h \nu \Delta \nu}{\sigma}
$$

From Eq. (2.3), we see that the highest peak powers require both a small emission cross section and a large spectral bandwidth.

The greatest obstacle in any gain medium for ultra-short pulses, in truth any medium through which the pulse must propagate, is the intensity dependent index of refraction $n=n_{0}+n_{2} I$, where $I$ is the intensity of the pulse. The intensity must remain at all times below the critical level at which nonlinear effects distort the spatial and temporal profiles of the pulse. The integrated nonlinear index of any material 
along the optical path of the laser is given by the $B$ integral

$$
B=\frac{2 \pi}{\lambda} \int \frac{\Delta n}{n} d l=\frac{2 \pi}{\lambda} n_{2} \int_{0}^{L} I(z) d z
$$

where $\lambda$ is the laser-wavelength and $L$ is the propagation length. Critical intensities correspond to a $B$ value of approximately $2 \pi$. These nonlinear distortions can cause severe effects such as filamentation and self focusing, resulting in damage to the amplifier. This is the most challenging issue in the amplification of USPs because it shows that a pulse as short as a picosecond with a fluence of $1 \mathrm{~J} / \mathrm{cm}^{2}$ could travel only $1 \mathrm{~mm}$ through a standard solid state laser amplifier before nonlinear distortions would degrade the beam.

The advent of chirped-pulse amplification (CPA) introduced a technique for attaining peak powers while maintaining a low value of the $B$ integral within the gain medium $[11,16,17]$. A chirped-pulse simply means a pulse whose electromagnetic frequency varies with time. In this technique, USPs are stretched temporally before amplification so that the pulse intensity is low in the gain medium, and then recompressed after the desired level of amplification. Fig. 2.1 shows a schematic of a modern stretcher-compressor system that uses holographic gratings for both processes of stretching and compressing.

In the diagram, a sub-picosecond pulse is generated in the oscillator and enters the laser-chain from the left. It is immediately deflected into the stretcher, where the opposed holographic gratings induce a positive group velocity dispersion (GVD) such that the red components of the pulse travel a shorter distance through the system 


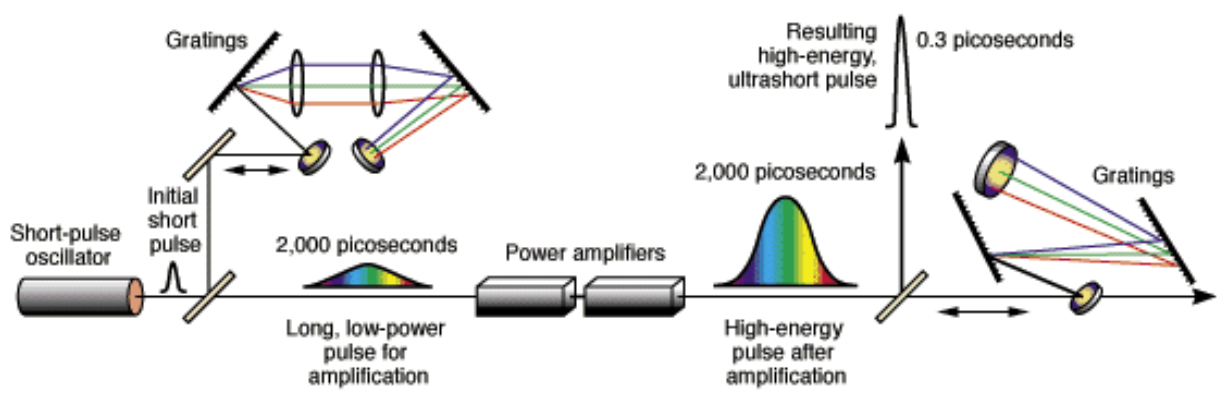

Figure 2.1: Diagram of stretcher-compressor system for CPA.

than the blue components. Once the pulse is stretched to several nanoseconds in length, it is safe to amplify in standard solid state amplifiers. After amplification, the high-energy pulse is compressed by parallel gratings back down to sub-picosecond pulse length.

After a pulse has been amplified and compressed, it must be focused to achieve a high intensity. The smallest theoretical focal spot diameter (measured by $\frac{1}{e^{2}}$ points of the spatial intensity distribution) for a laser-pulse with a Gaussian profile is

$$
d_{1 / e^{2}}=2.44 \lambda f / \#
$$

where $f$ /\# represents the f-number (diameter / focal length) of the final focusing optic and $\lambda$ is the wavelength of the laser light. Ideally this spot size would be limited only by diffraction to the smallest possible size known as the diffraction limit. In practice, however, passive optical imperfections and thermal effects due to non-uniform pumping of the gain medium limit the smallest achievable focused spot diameter to within 2-3 times the diffraction limit. 
Although chirped-pulses can be safely amplified in standard solid-state laser materials, peak power achievable with CPA is limited by the damage threshold of the diffraction gratings. Gold diffraction gratings have a damage threshold of approximately $400 \mathrm{~mJ} / \mathrm{cm}^{2}$. In order to protect the gratings, most compressors are typically operated at $75 \%$ of the damage threshold or approximately $300 \mathrm{~mJ} / \mathrm{cm}^{2}$. Therefore, the limiting factor in the energy up to which a pulse can be amplified is the size of the compressor gratings. The first petawatt laser [18] developed at Lawrence Livermore National Laboratory (LLNL) used $94 \mathrm{~cm}$ diameter large-aperture diffraction gratings (1480 lines/mm) with a throughput efficiency of $84 \%$ of the initial energy. The system was operated at a damage threshold of $330 \mathrm{~mJ} / \mathrm{cm}^{2}$, which limited the incident beam energy to $880 \mathrm{~J}$.

The LLNL petawatt laser employed a Ti:sapphire oscillator to produce the shortest possible pulses. However, since it also relied on the high power Nd:glass amplifier chain of the NOVA laser, the recompressible pulse length was much longer than the initial pulse due predominately to the effects of gain narrowing. In the amplifying material, a pulse exhibiting a Gaussian line shape, frequency bandwidth is reduced as described by,

$$
\Delta \omega=\Delta \omega_{A m p} \sqrt{\frac{3}{G\left(\omega_{A m p}\right)-3}}
$$

where $\omega_{A m p}$ is the material gain bandwidth and $G$ is the gain in decibels. Systems with high gain and a small gain bandwidth such as Nd:glass, will tend to decrease the frequency bandwidth of the propagating pulse. A loss of frequency bandwidth makes 
it impossible to compress a pulse back to its original sub-picosecond pulse length.

In the case of the petawatt laser, the pulse immediately after the stretcher had a pulse duration of $\sim 3 \mathrm{~ns}$ and a bandwidth of $16.5 \mathrm{~nm}$. After the final stage of glass amplifiers, gain narrowing had reduced the pulse to a pulse duration of $900 \mathrm{ps}$ and a spectral width of $5.3 \mathrm{~nm}$. This limited the recompression of a pulse to $\sim 500 \mathrm{fs}$. At the typical operating energy of $660 \mathrm{~J}$, this allowed for the production of pulses with a peak power was $1.2 \mathrm{PW}$.

Systems that use a large chain of glass amplifiers can achieve high energy and peak power, however, they suffer from incredibly poor contrast, the ratio of the prepulse to the main pulse intensity. The main sources of prepulse in any laser system are: (1) pulses leaking from round trips in the regenerative amplifier; (2) amplified spontaneous emission (ASE) from each stage of the amplification chain; (3) high-order phase distortions, typically due to system bandwidth limitations and/or stretching or compression errors. Although prepulse is not a main concern in many applications, in the study of solid density plasmas, prepulse will greatly affect the density conditions of the plasma that interacts with the main pulse. The LLNL Petawatt had a prepulse that arrived at the target 2 ns before the main pulse with a contrast of $10^{-4}$. This prepulse created a substantial preplasma in front of the target surface.

The JanUSP laser at LLNL is a state-of-the-art short-pulse, high contrast laser system and currently holds the world record for highest irradiance at $I \lambda^{2}>10^{21} \mathrm{~W} \mu \mathrm{m}^{2} / \mathrm{cm}^{2}$. Its name derives from the "Janus" Nd:glass laser system that is used as a pump source 
for the final two stages of an ultra-short-pulse (USP) laser system [19, 20, 21]. A schematic of the JanUSP facility is shown in Fig. 2.2.

The JanUSP laser utilizes several technological advancements in laser and material science. First and foremost, the final two amplifiers in the system are $10 \mathrm{~cm}$ diameter Ti:sapphire disks with a thickness of $2.5 \mathrm{~cm}$, making them the largest Ti:sapphire disk amplifiers ever produced. These are pumped by the large aperture, Nd:glass, Janus laser that is frequency doubled to $\sim 530 \mathrm{~nm}$, green light, near the titanium absorption peak. With $150 \mathrm{~J}$ of green light pumping the amplifier disks, as high as $15 \mathrm{~J}$ of $800 \mathrm{~nm}$ light has been achieved on target. Since the amplifiers are laser-pumped instead of pumped by flash lamps, less energy is wasted in the form of heating the gain medium. This decreases the effects of thermal lensing, which can distort the beam, and reduces the cooling time between shots.

After amplification, compression of the laser pulse is performed with two 1480 line/mm gold coated, large aperture $(G 1=33 \mathrm{~cm}$ and $G 2=40 \mathrm{~cm})$, diffraction gratings, with an overall throughput efficiency of $75 \%$ in double pass. Assuming a Gaussian distribution the final pulse width is $\sim 75 \mathrm{fs}$, and the FWHM spectral width is about $13.6 \mathrm{~nm}$ which is nearly identical to that of the initial mode-locked oscillator pulse. This gives a time-bandwidth product of $\Delta \nu \Delta \tau=0.48$.

After the pulse is compressed, it is routed to the target chamber, in the same $10^{-5}$ torr range vacuum environment as that of the compressor. It is focused onto the target by a $15.24 \mathrm{~cm}$ diameter gold-coated $f / 2$ off-axis parabola. The FWHM of the 


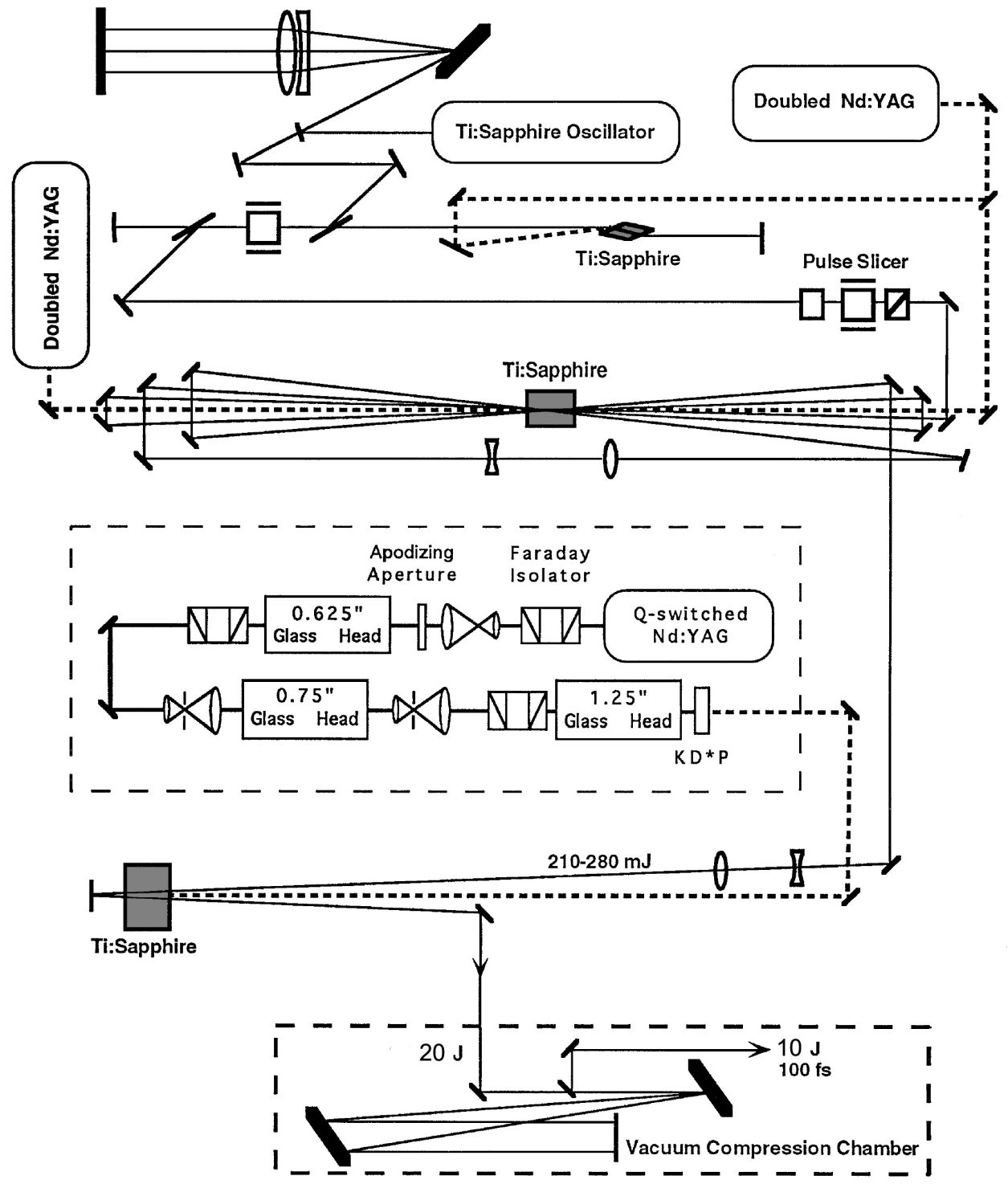

Figure 2.2: Diagram of the JanUSP laser system including the frequency-doubled Nd:glass laser used to pump the final amplifiers. Used with permission from D. Price [20]. 
focal spot has been measured to be $3.2 \mu \mathrm{m}$ or about 1.5 times the diffraction limit. It is this combination of energy $>10 \mathrm{~J}$, pulse width $<100 \mathrm{fs}$ and a focal spot size of 1.5 times the diffraction limit that make possible an irradiance $>10^{20} \mathrm{~W} \mu \mathrm{m}^{2} / \mathrm{cm}^{2}$.

\begin{tabular}{ccc}
\hline \hline & JanUSP (Ti:sapphire) & Petawatt (Nd:glass) \\
& & \\
Energy $(\mathrm{J})$ & 10 & $\sim 600$ \\
$\Delta \tau(\mathrm{fs})$ & 100 & $500-1000$ \\
$\lambda(\mathrm{nm})$ & 800 & 1053 \\
Power $(\mathrm{TW})$ & 100 & 1200 \\
$I \lambda^{2}\left(\mathrm{~W} \mu \mathrm{m}^{2} / \mathrm{cm}^{2}\right)$ & $>10^{20}$ & $8 \times 10^{20}$ \\
Contrast & $10^{-9}$ & $10^{-4}$ \\
& & \\
\hline \hline
\end{tabular}

Table 2.2: Comparison of the LLNL JanUSP and Petawatt laser system characteristics.

Shorter pulses, fewer amplifiers in the laser chain, and various techniques of prepulse reduction near the oscillator give JanUSP another advantage over glass laser system in the form of very high contrast. The focused prepulse has been measured to be no greater than $10^{11} \mathrm{~W} / \mathrm{cm}^{2}$. A saturable absorber [22] before the regenerative amplifier combined with two stages of good quality crossed-glan laser polarizers and Pockel cells after the regenerative amplifier effectively attenuate the prepulse, providing a contrast of $10^{-9}$. This ensures that the main pulse will interact predominately with the target at solid density conditions, near the target surface instead of being absorbed in a preplasma hundreds of microns in front of the original target surface.

For completeness, the characteristics of both the JanUSP Ti:sapphire and the 
Petawatt Nd:glass laser systems have been listed in Table 2.2. We can see that peak power is somewhat more attainable with the large energies available in glass laser systems, while high irradiance is achievable with both Ti:sapphire and Nd:glass systems. Due to both its high irradiance and high contrast, the JanUSP laser system is an excellent tool for the study of ultra-intense laser-matter interactions at solid density, and was the principle system used for the research presented in this thesis.

\subsection{Absorption Mechanisms}

\subsubsection{Fundamental Plasma Parameters}

An absorption mechanism is a physical process (or collection of processes) that result in the transfer of energy from the laser pulse to the solid material or plasma with which the pulse is interacting. In the case of most classical or long-pulse lasermatter interactions, absorption predominately takes place in the long scale-length density plasma in front of the target. Once the intensity of the laser pulse exceeds $10^{12} \mathrm{~W} / \mathrm{cm}^{2}$, the material begins to ionize, and a plasma is formed on the surface of the target.

On many short-pulse laser systems, the prepulse that precedes the main pulse has sufficient intensity to induce ionization. The main, high intensity laser pulse (when it arrives typically nanoseconds later) interacts with this "preplasma" in front of the 
target surface. The density scale-length of the preplasma is defined as,

$$
L_{n} \equiv \frac{n}{d n / d x} \approx c_{s} \tau_{\text {Lped }},
$$

where $n$ is the plasma density, $d n / d x$ is the slope of the density, $\tau_{\text {Lped }}$ is the duration of the laser-pulse pedestal, and $c_{s}$ is the ion sound speed given by,

$$
c_{s}=\sqrt{k_{B}\left(Z T_{e}+T_{i o n}\right) / M_{i o n}} .
$$

Depending on the contrast of the laser system, the pedestal can be anywhere from $10^{-4}$ to $10^{-9}$ times the intensity of the main pulse. Short pulse lasers with a pedestal of $10^{-8}$ or less of the main pulse intensity tend to have short, front surface scale-length plasmas $(2-5 \mu \mathrm{m})$. Lasers with a pedestal in the $10^{-4}$ range tend to generate long scale-length preplasmas that extend up to hundreds of microns in front of the target surface. For the case of USPs, if the pre-pulse or pedestal as it is sometimes called can be reduced, the main pulse energy can interact with a high-density plasma near the original target surface.

Laser light transfers its energy to the plasma, primarily, through the electrons that oscillate in the electric field of the laser pulse as described by,

$$
\frac{p_{o s c}}{m_{e} c}=\frac{\gamma v_{o s c}}{c}=\frac{e E_{o}}{m_{e} c \omega_{o}}=\sqrt{\frac{I \lambda^{2}}{1.37 \times 10^{18}}},
$$

where $p_{o s c}$ and $v_{o s c}$ are the electron oscillation momentum and velocity respectively, $e$ is the electron charge, $E_{o}$ is the laser electric field, $I \lambda^{2}$ is the irradiance in units of $\mathrm{W} \mu \mathrm{m}^{2} / \mathrm{cm}^{2}$ [23]. For an irradiance $>10^{18} \mathrm{~W} \mu \mathrm{m}^{2} / \mathrm{cm}^{2}$, the quiver energy of the 
electron in the electric field of the laser pulse exceeds the rest mass of $0.511 \mathrm{MeV} / \mathrm{c}^{2}$. This requires the consideration of relativistic effects when describing the coupling of ultra-short, ultra-intense laser-pulses to a plasma.

If we ignore collisional effects, the propagation of a laser pulse (or any electromagnetic wave) in a plasma is governed by the dispersion relation

$$
k\left(\omega_{o}\right)=\frac{1}{c} \sqrt{\omega_{o}^{2}-\frac{\omega_{p e}^{2}}{\gamma}}
$$

where $k\left(\omega_{o}\right)$ is the wavenumber of the laser, $\gamma$ is the relativistic factor, $\omega_{o}$ is the frequency of the laser light and $\omega_{p e}=\sqrt{n e^{2} / \epsilon_{o} m_{e}}$ is the plasma frequency in m.k.s units. In the non-relativistic case $(\gamma=1)$, which is valid up to an irradiance of $5 \times 10^{17} \mathrm{~W} \mu \mathrm{m}^{2} / \mathrm{cm}^{2}$, the wave will propagate through the increasingly denser plasma up to the point at which $\omega_{p e}=\omega_{o}$. At this point, the wavenumber becomes imaginary and the wave can no longer propagate. The density at which this occurs is known as the critical density and is given simply by solving for the density in the definition of the plasma frequency where $\omega_{p e}$ is equal to the frequency of the laser light,

$$
\omega_{o}^{2}=\frac{n_{c r} e^{2}}{\gamma m_{e}^{o} \epsilon_{o}},
$$

which leads directly into

$$
n_{c r}=\frac{\omega_{o}^{2} \gamma m_{e}^{o} \epsilon_{o}}{e^{2}}
$$

where $m_{e}^{o}$ represents the electron rest mass. Plugging the constants into the above equation yields the critical density in the form

$$
n_{c r}=\gamma \frac{1.1 \times 10^{21}}{\lambda_{\mu}^{2}} \quad \text { in units of } \mathrm{cm}^{-3}
$$


where $\lambda_{\mu}$ is the laser wavelength in microns.

At the critical surface, the plasma density is sufficiently high for the electrons oscillating in the electric field of the laser to compensate the incident field and reradiate the light wave. While the energy contained in the laser pulse is reflected, an evanescently decaying field is transmitted across the barrier. The length to which this field extends into the over-dense plasma is known as the plasma skin depth, given by $c / \omega_{p e}$.

We can see from Eq. (2.13), in the relativistic case, the critical density is increased by the factor $\gamma$, which allows the laser to penetrate deeper into the plasma. This occurs due to the relativistic mass increase of the electrons oscillating in the electric field of the laser. This augmented mass reduces the electrons ability to react as quickly to the oscillating electric field and, therefore, a higher density is required to generate the same compensating field as in the non-relativistic case.

\subsubsection{Inverse Bremsstrahlung}

As the laser pulse propagates into the plasma, up to the critical surface, it loses energy. One of the most well understood, and the most dominant absorption mechanism for classical, long pulse laser systems is inverse bremsstrahlung. An electromagnetic wave incident on one electron cannot impart any energy since the electron will experience a net zero acceleration in one full cycle of the electric field. In a plasma, however, stochastic effects of other charges will cause collisions, preventing electrons 
from making complete cycles within the experienced electric filed. The laser pulse will diminish as its energy is transferred to the electrons and the electron-plasma temperature increases. The electrons, in turn, couple to the ions, thereby increasing the ion-plasma temperature.

\subsubsection{Resonant Absorption}

Another important absorption mechanism in the regime of long-pulse systems occurs near the critical surface, as electron-plasma waves are resonantly excited and driven into the over-dense plasma. This form of resonant absorption is greatest when the laser pulse is obliquely incident on a target at some angle, $\theta$ as shown in Fig. 2.3. At oblique incidence, the laser pulse cannot propagate up the critical surface but rather up to the density known as the turning point, given by

$$
n_{\text {turn }}=n_{c r} \cos ^{2} \theta,
$$

where is it specularly reflected. Some of the energy carried in the laser pulse will tunnel into the over-dense plasma, and in the case of p-polarized light (where the electric field of the laser light is in the direction of the density gradient), can resonantly excite electron plasma waves. It has been shown that electrons accelerated by this process have a distribution that is roughly a high energy tail superimposed on the initial background Maxwellian temperature distribution [24], and is observed to scale as

$$
T_{h o t} \approx 10\left(T_{k e V} I_{15} \lambda^{2}\right)^{1 / 3} k e V,
$$




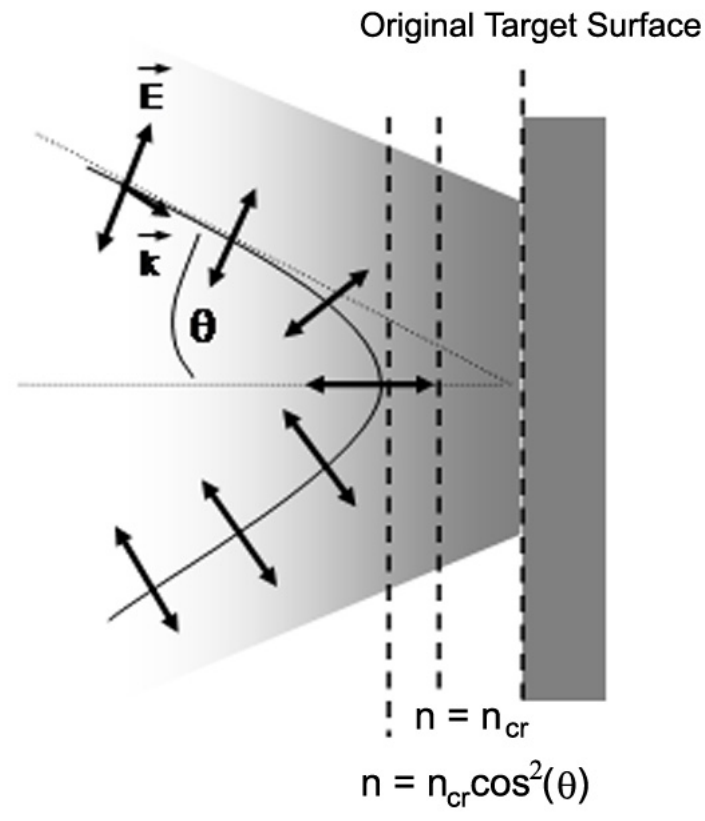

Figure 2.3: P-polarized light at oblique incidence will be reflected at the turning point rather than the critical surface.

where $T_{k e V}$ is the background electron temperature in $k e V$, and $I_{15} \lambda^{2}$ is the irradiance in units of $10^{15} \mathrm{~W} \mu \mathrm{m}^{2} / \mathrm{cm}^{2}$.

These two "classical" absorption mechanisms, along with various three-wave instabilities such as Stimulated Raman Scattering (SRS) and Stimulated Brillioun Scattering (SBS), will dominate absorption processes up to intensities of $10^{17} \mathrm{~W} / \mathrm{cm}^{2}$. However, as the electron oscillation velocity becomes relativistic $\left(\frac{p_{o s c}}{m_{e} c} \approx 1\right)$, inverse bremsstrahlung and resonant absorption are dwarfed by new absorption mechanisms that turn on at intensities $>10^{18} \mathrm{~W} / \mathrm{cm}^{2}$. 


\subsubsection{Vacuum Heating}

"Not-so-resonant" resonance absorption, also known as vacuum heating, becomes very significant in the regime of ultra-short pulses with high intensities. Vacuum heating is similar to the "classical" resonance absorption mechanism in that electrons are driven across a density gradient by the electric field of the laser pulse. The difference between the two arises in the fact that unlike resonance absorption, where the laser light interacts with an increasing plasma density with scale length many times the laser wavelength, vacuum heating requires the density gradient scale length to be less than that of the laser wavelength. In this scenario, no resonance can exists. Heating occurs as electrons at the vacuum plasma interface are pulled into vacuum by the incident electric field and then driven back into the plasma at a random phase.

In multiterawatt-laser-plasma interactions, dominant absorption mechanisms tend to be a combination of resonance absorption, vacuum heating and $\vec{J} \times \vec{B}$ absorption due to the oscillating component of the ponderomotive force of the laser light as described below.

\subsection{The Ponderomotive Force}

\subsubsection{Overview and Historical Background}

The ponderomotive (or nonlinear) force is always present in a spatially varying force field that is oscillating in time. It can be derived in various ways starting from 
principles of electrodynamics, hydrodynamics, and plasma physics. In 1845, Lord Kelvin first described the ponderomotive force as a force density $F_{K}$ produced in a dielectric medium of refractive index $\tilde{n}$ given by

$$
F_{K}=\vec{P} \cdot \nabla \vec{E}
$$

where $\vec{P}$ is the polarization of the material, $\vec{E}$ is the electric field, and $\tilde{n}^{2}=\epsilon$ is the dielectric constant [25]. Remembering the definition of polarization, $\vec{P}=\left(\tilde{n}^{2}-1\right) \epsilon_{o} \vec{E}$ and using the vector identity,

$$
\vec{E}_{s} \times\left(\nabla \times \vec{E}_{s}\right)=\frac{1}{2} \nabla\left(\vec{E}_{s} \cdot \vec{E}_{s}\right)-\left(\vec{E}_{s} \cdot \nabla\right) \vec{E}_{s}
$$

we can rewrite Eq.(2.16) as

$$
F_{K}=\epsilon_{o}\left(\frac{\tilde{n}^{2}-1}{2}\right) \nabla E^{2}-\epsilon_{o}\left(\tilde{n}^{2}-1\right) \vec{E} \times(\nabla \times \vec{E}) .
$$

In electrostatics, the electric field can be defined as the curl-free gradient of a potential $\psi$,

$$
\vec{E}=-\nabla \psi ; \quad \nabla \times \vec{E}=0 .
$$

Therefore, in the electrostatic case, the last term in Eq. (2.18) is zero, and the ponderomotive force is equivalent to the gradient of $\vec{E} \cdot \vec{E}$,

$$
F_{K}=\epsilon_{o}\left(\frac{\tilde{n}^{2}-1}{2}\right) \nabla E^{2}
$$

In the case of a plasma, the refractive index $\tilde{n}$ is given in terms of the plasma frequency $\omega_{p e}$ as,

$$
\tilde{n}^{2}=1-\frac{\omega_{p e}^{2}}{\omega_{o}^{2}}
$$


Substituting the refractive index into Eq. (2.20) we can describe the ponderomotive force of Kelvin acting on a plasma as the force density,

$$
F_{K}=-\frac{1}{2} \frac{\omega_{p e}^{2}}{\omega_{o}^{2}} \epsilon_{o} \nabla E^{2}
$$

\subsubsection{Single-Particle Derivation of the Ponderomotive Force}

We can derive the ponderomotive force of an electromagnetic (EM) wave in a plasma by considering the motion of an electron in the oscillating $\vec{E}$ and $\vec{B}$ fields of the wave [1]. The electron equation of motion in an EM wave is given by the generalized Lorentz force,

$$
m \frac{d \vec{v}}{d t}=-e[\vec{E}(\vec{r})+\vec{v} \times \vec{B}] .
$$

We assume the electric field has the waveform

$$
\vec{E}(\vec{r})=\vec{E}_{s}(\vec{r}) \cos \left(\omega_{o} t\right),
$$

where $\vec{E}_{s}(\vec{r})$ contains the spatially varying amplitude. In the first order, we can neglect the $\vec{v} \times \vec{B}$ term and consider the electric field at the initial electron position $\vec{r}_{o}:$

$$
\begin{gathered}
m \frac{d \vec{v}_{1}}{d t}=-e \vec{E}\left(\vec{r}_{o}\right), \\
\overrightarrow{v_{1}}=-\left(\frac{e}{\omega_{o} m_{e}}\right) \overrightarrow{E_{s}} \sin \left(\omega_{o} t\right)=\frac{d \vec{r}_{1}}{d t},
\end{gathered}
$$




$$
\delta \vec{r}_{1}=\left(\frac{e}{\omega_{o}^{2} m_{e}}\right) \vec{E}_{s} \cos \left(\omega_{o} t\right) .
$$

$\delta \overrightarrow{r_{1}}$ is the instantaneous displacement of the electron in the electric field. Going to second order, we expand $\vec{E}_{s}(\vec{r})$ about $\vec{r}_{o}$,

$$
\vec{E}(\vec{r})=\vec{E}\left(\vec{r}_{o}\right)+\left.\left(\delta \vec{r}_{1} \cdot \nabla\right) \vec{E}\right|_{r=r_{o}} .
$$

We must now consider the second order $\vec{v}_{1} \times \vec{B}_{1}$ term. Using Maxwell's equations we can derive $\vec{B}_{1}$ in terms of the electric field:

$$
\begin{gathered}
\nabla \times \vec{E}=-\frac{\partial \vec{B}}{\partial t}, \\
\vec{B}_{1}=-\left(\frac{1}{\omega}\right) \nabla \times \vec{E}_{s} \sin \left(\omega_{o} t\right) .
\end{gathered}
$$

From this, we can see the second order contribution to Eq.(2.23) is,

$$
m_{e} \frac{d \vec{v}_{2}}{d t}=-e\left[\left(\delta \vec{r}_{1} \cdot \nabla\right) \vec{E}+\vec{v}_{1} \times \vec{B}_{1}\right] .
$$

Inserting Eqs. (2.26), (2.27), (2.28) and (2.30) into (2.31) and averaging over time, gives us

$$
m_{e}\left\langle\frac{d \vec{v}_{2}}{d t}\right\rangle=-\frac{e^{2}}{m_{e} \omega_{o}^{2}} \frac{1}{2}\left[\left(\vec{E}_{s} \cdot \nabla\right) \vec{E}_{s}+\vec{E}_{s} \times\left(\nabla \times \vec{E}_{s}\right)\right]=\vec{f}_{N L} .
$$

The factor $\frac{1}{2}$ arises from the time averaging of the sine term, $\left\langle\sin ^{2}\left(\omega_{o} t\right)\right\rangle=\left\langle\cos ^{2}\left(\omega_{o} t\right)\right\rangle=$ $\frac{1}{2}$. Again we make use of the double cross product identity to cancel the $\left(\vec{E}_{s} \cdot \nabla\right) \vec{E}_{s}$ term, which leaves us with,

$$
\vec{f}_{N L}=-\frac{1}{4} \frac{e^{2}}{m_{e} \omega^{2}} \nabla E_{s}^{2}
$$


However, this is only the force on a single electron. The force acting on the plasma is this force times the density $n$ expressed in terms of the plasma frequency $\omega_{p e}$. Finally, realizing that $E_{s}^{2}=2\left\langle E^{2}\right\rangle$, we arrive at the standard nonlinear force,

$$
\vec{F}_{N L}=-\frac{1}{2} \frac{\omega_{p e}^{2}}{\omega_{o}^{2}} \nabla\left\langle\epsilon_{o} E^{2}\right\rangle .
$$

If the wave generating the ponderomotive force is an electrostatic wave, the changing electron density will generate variations in the electric field and the first term in Eq. (2.31) will dominate. The physical mechanism in this case is that electrons oscillating along the direction of the electric field move further in the half-cycle when they are moving from a strong-field region to a weak field region, than when they are moving from a weak-field region into a strong field region. Therefore there is a net drift away from the region of strong field.

\subsubsection{Examples of the Nonlinear Force}

As an EM wave propagates through a plasma in the direction of increasing density, the magnitude of the electric field will "swell" in a manner inversely proportional to the dielectric constant. We can derive this relationship very easily by beginning with the definition of Poynting's vector:

$$
\begin{aligned}
\vec{S} & =\frac{1}{\mu_{o}}(\vec{E} \times \vec{B}) \\
|\vec{S}| & =\frac{1}{\mu_{o}}|\vec{E}||\vec{B}| \quad\left(\text { from Maxwell's eqs: } k|\vec{E}|=\omega_{o}|\vec{B}|\right) \\
|\vec{S}| & =\frac{k}{\mu_{o} \omega_{o}}|\vec{E}|^{2}
\end{aligned}
$$




$$
|\vec{S}|=\frac{1}{\mu_{o} c^{2}} \frac{k c^{2}}{\omega_{o}}|\vec{E}|^{2} .
$$

Using the plasma formulation of $\frac{k c^{2}}{\omega_{o}}$,

$$
\frac{k c^{2}}{\omega_{o}}=\frac{c}{\omega_{o}} \sqrt{\omega_{o}^{2}-\omega_{p e}^{2}}=c \sqrt{1-\frac{\omega_{p e}^{2}}{\omega_{o}^{2}}}=c \sqrt{\epsilon},
$$

we can rewrite the Poynting vector as,

$$
|\vec{S}|=\frac{c \sqrt{\epsilon}}{\mu_{o} c^{2}}|\vec{E}|^{2} .
$$

Ignoring the dissipation of energy through collisions, the energy carried by the wave in vacuum must remain equal to the energy carried by the wave in the plasma

$$
|\vec{S}|_{\text {vac }}=|\vec{S}|_{\text {plasma }} .
$$

Therefore, the electric field of an EM wave in a plasma can be expressed in terms of the vacuum electric field $\vec{E}_{v a c}$ and the plasma dielectric constant $\epsilon$ as,

$$
|\vec{E}|^{2}=\frac{\left|\vec{E}_{v a c}\right|^{2}}{\sqrt{\epsilon}} .
$$

Fig. 2.4 shows a calculation of the square of the electric field of an EM plane wave as it propagates through a plasma. The square of the field slowly increases until it reaches a singularity at the critical surface. Beyond the critical surface - where the wave number becomes imaginary - the wave can no longer propagate and becomes a rapidly decaying electric field. The resulting nonlinear forces effectively ablate the plasma in the under-dense region (driving the plasma corona toward vacuum), and compress the plasma in the over-dense region. This compression is equivalent to the classical radiation pressure at the critical surface. 


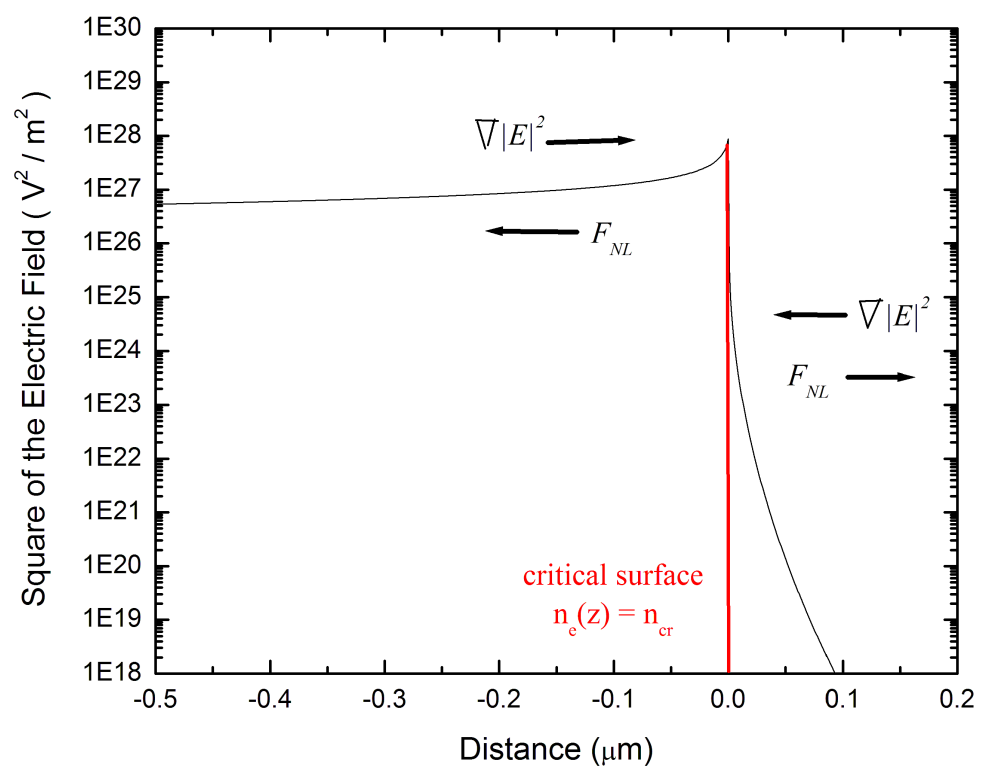

Figure 2.4: The nonlinear forces act in the direction opposite the $E^{2}$-field, ablating the under-dense while compressing the over-dense. Calculation performed in Mathematica presuming mono-chromatic plane wave $\left(\vec{E}=E_{o} e^{i k z}\right)$ with laser intensity of $I=10^{19} \mathrm{~W} / \mathrm{cm}^{2}$ and laser wavelength $\lambda=1 \mu \mathrm{m}$.

Self-focusing of laser pulses is also an effect of the nonlinear force. Most laser focal spots have a transverse Gaussian intensity distribution that results in a radially directed ponderomotive force. As particles are expelled from regions of high $\left\langle E^{2}\right\rangle$, the refractive index in these regions increases, forcing the plasma to act as a convex lens, causing the laser light. The threshold laser power (in gigawatts) for which this effect becomes significant is given by,

$$
P_{\text {threshold }}=17 \frac{n_{c r}}{n} \mathrm{GW}
$$

Self-focusing of the laser pulse will result in a higher intensity, which could produce other nonlinear effects that are intensity dependant. 


\subsection{4 $\vec{J} \times \vec{B}$ Heating}

As stated above, intensities $>10^{18} \mathrm{~W} / \mathrm{cm}^{2}$ can now routinely be achieved. In fact, intensities $>10^{20} \mathrm{~W} / \mathrm{cm}^{2}$ have already been demonstrated [19]. Self-focusing of the laser light in the plasma can effectively raise these intensities even higher. If the quiver velocity of an electron in the laser pulse becomes relativistic, it is the second term in Eq. (2.31) that dominates the ponderomotive force. As the $\vec{v} \times \vec{B}$ term becomes equal to the electro-static term, the electron orbit is distorted and the electron is pushed in the direction of propagation. This longitudinal component of the ponderomotive force can dephase electrons from what would otherwise be a purely transverse oscillation, into an orbit resembling a figure-eight.

At this level of ultra-intense laser-plasma interactions, we must use the relativistically correct expression for the ponderomotive force [26],

$$
\vec{F}_{N L, r e l}=-\nabla\left\{(\gamma-1) m_{0} c^{2}\right\}
$$

where the relativistic factor is defined as $\gamma=\sqrt{1+\frac{p^{2}}{m_{0}^{2} c^{2}}}$. Although this force is typically non-resonant in the direction of propagation, at the critical surface where the wave becomes a strong, rapidly decaying field, it can accelerate electrons to very high energies. For a linearly polarized wave, the force has a frequency component of $2 \omega_{0}$. This means that twice in one laser cycle, electrons are accelerated into the over-dense plasma.

Ponderomotive heating, also referred to as $\vec{J} \times \vec{B}$ heating arises due to the nonlinear 
component of the Lorentz force, and is similar to the "not-so-resonant" resonance absorption mechanism discussed above. It differs in that the oscillating component of the ponderomotive force is driving electrons across the critical surface rather than the component of the electric field of the laser, as in the vacuum heating mechanism.

We can calculate a temperature for the hot electrons accelerated by $\vec{J} \times \vec{B}$ heating by assuming it is roughly equal to the ponderomotive potential [27],

$$
U=\int \vec{F}_{N L, r e l} \cdot d x .
$$

Therefore, the electron temperature can be described by,

$$
T_{H o t}=(\gamma-1) m_{0} c^{2} .
$$

Plugging in for $\gamma$ gives us,

$$
T_{H o t}=\left(\sqrt{1-\frac{p^{2}}{m_{0}^{2} c^{2}}}-1\right) m_{0} c^{2} .
$$

We can further simplify the expression for $T_{H o t}$ by substituting our expression for the electron oscillation momentum from Eq. (2.9) into Eq. (2.47), which results in

$$
T_{\text {Hot }}=\left[\sqrt{1-\frac{I \lambda^{2}}{1.37 \times 10^{18}}}-1\right] m_{0} c^{2} .
$$

We can see from Eq. (2.48), as irradiance exceeds $10^{18} \mathrm{~W} \mu \mathrm{m}^{2} / \mathrm{cm}^{2}$, electron temperatures of several $\mathrm{MeV}$ become possible, as will be discussed in the next chapter. 


\section{Chapter 3}

\section{Ion Acceleration}

Ion acceleration from laser-plasma interactions has been studied experimentally, theoretically and computationally for the past thirty years. In this chapter, we present theoretical descriptions of ion acceleration as well as a review of ion acceleration experiments in the classical, long-pulse and contemporary, short-pulse regimes.

\subsection{Early Ion Acceleration Research}

Initially, the acceleration of ions from laser plasmas was seen only as a deleterious effect to target compression in laser-fusion experiments. In such experiments, recoil from the laser ablation of ions drives the compression of spherical shells of various compositions to high densities and temperatures. Lasers used in conventional laserfusion studies are typically high energy (several kJ) and long pulse duration (several ns), with intensities on order of $I \approx 10^{15} \mathrm{~W} / \mathrm{cm}^{2}$. During ablation, ions and electrons 
in the plasma absorbed energy from the laser pulse via the mechanisms described in Chapter 2. The majority of electrons establish a thermal equilibrium with the ions at a plasma temperature of $2-3 \mathrm{keV}$, which drives the ablation process. However, if sufficient energy remains in the laser pulse at the time it reaches the critical surface, a small fraction of electrons can be accelerated to energies up to tens of $\mathrm{keV}$ due to ponderomotive processes and enhanced resonant absorption. These "hot" electrons are often undesirable in ICF experiments since they can propagate through the target preheating the fusion fuel, exacerbating the difficulties associated with compression.

Hot electrons expanding toward vacuum slow down, and are eventually pulled back by the potential of the laser-target which has become strongly, positively charged. As these electrons propagate toward the critical surface, they are reflected out into vacuum once more, and the process repeats itself many times over the duration of the laser pulse. As the electrons circulate through the target, however, the laser electric field is decreasing. This results in the electrons receiving a net acceleration away from the target toward vacuum. This net acceleration toward vacuum couples to the ions in the form of the self-consistent electric field. This coupling mechanism of laser light to electrons to high energy ions can decrease the energy available for compression and heating of the laser target.

Early analytical efforts to describe the the expansion of a plasma into vacuum, employed a one-dimensional fluid model with the assumption of quasi-neutrality [28]. In this model, the electrons are assumed to be in isothermal equilibrium with a density 
given by the Boltzmann relation,

$$
n_{e}=n_{e o} e^{\left(e \phi / T_{e}\right)} .
$$

If quasi-neutrality is assumed $\left(n_{e}=Z n_{i}\right.$ where $n_{e}$ and $n_{i}$ are the electron and ion densities respectively), and electron inertia is neglected, the ions can be described by the continuity and momentum equations,

$$
\begin{aligned}
& \frac{\partial n_{i}}{\partial t}+\frac{\partial}{\partial x}\left(n_{i} v_{i}\right)=0, \\
& \frac{\partial v_{i}}{\partial t}+v_{i} \frac{\partial v_{i}}{\partial x}=-\frac{c_{s}^{2}}{n_{i}} \frac{\partial n_{i}}{\partial x},
\end{aligned}
$$

where $v_{i}$ and $n_{i}$ are the ion velocity and density, respectively, and $c_{s}$ is the ion sound speed.

We can obtain self-similar solutions to Eq. (3.2) in planar geometry by assuming $v_{i}$ and $n_{i}$ have dependence on space and time of the form $\xi=x / t$, which gives the usual solutions:

$$
\begin{aligned}
& n_{i}=n_{o} e^{-\left(1+x / c_{s} t\right)}, \\
& v_{i}=c_{s}+x / t .
\end{aligned}
$$

A rough estimate for the accelerating electric field can be found from the electron momentum equation near the ion front at a snap-shot in time:

$$
\begin{aligned}
n_{e} e \vec{E} & =\frac{\partial P_{e}}{\partial x}, \\
\vec{E} & =\frac{k_{B} T_{e} \partial n_{e} / \partial x}{n_{e} e},
\end{aligned}
$$


where $P_{e}=n k_{B} T_{e}$ is the plasma pressure, and $e$ is the electron charge. If we take the derivative of $n_{e}$,

$$
\frac{\partial n_{e} / \partial x}{n_{e}}=-\frac{1}{c_{s} t},
$$

and realize that in the case of the self-similar solution, $c_{s} t=L_{n}$, where $L_{n}$ is the local plasma scale length, we can show the electric field is given by ,

$$
\vec{E}=\frac{k_{B} T_{e}}{e L_{n}} .
$$

Given high electron temperatures and small plasma scale lengths, the self-similar solution to the above equations predicts an electric field capable of accelerating ions to high energies. However, it also predicts an ion density profile which falls off to zero exponentially. In practice, the ion energy distribution does not extend to infinity, but rather reaches a maximum cutoff energy beyond which no ions are found. At the position of such an "ion front," the condition of quasi-neutrality is no longer satisfied and a self-similar solution is not possible [29]. Instead, the potential must be evaluated from the Poisson equation,

$$
\frac{\partial^{2} \phi}{\partial x^{2}}=\frac{e Z}{\epsilon_{o}}\left(n_{e o} e^{\left(e \phi / T_{e}\right)}-n_{i}\right) .
$$

The electric field at any point $x$ ahead of the ion front must be evaluated by integration of the charge density,

$$
\vec{E}(x)=\vec{E}(L)+\frac{1}{\epsilon_{o}} \int_{x_{o}}^{x} \rho\left(x^{\prime}\right) d x^{\prime},
$$

where $x_{o}$ denotes the position of the ion front. 


\subsubsection{The Los Alamos Experiments}

Experiments devoted purely to the study of fast-ion generation were performed at the Helios, $\mathrm{CO}_{2}$ laser facility of the Los Alamos National Laboratory (LANL) [30]. At that facility, pulses of 80-300 $\mathrm{J}$ with pulse duration of $1 \mathrm{~ns}$, could produce laser intensities on target of $I \approx 10^{15} \mathrm{~W} / \mathrm{cm}^{2}$. Due to the fundamental wavelength of $\mathrm{CO}_{2}$ lasers of $\lambda=10.6 \mu \mathrm{m}$, the irradiance $\left(I \lambda^{2}\right)$ is of order $10^{18} \mathrm{~W} \mu \mathrm{m}^{2} / \mathrm{cm}^{2}$ which is capable of producing hot electrons in the tens of $\mathrm{keV}$ range.

By employing a Thomson parabola, a device that separates ions by momentum as well as charge state, in combination with a cellulose-film particle track detector, the experimenters at LANL were able to observe velocity distributions of various ions. They showed that irradiating a plastic $\mathrm{CH}$ target produced protons with a maximum velocity of $\sim 10^{9} \mathrm{~cm} / \mathrm{s}$ and several charge states of carbon which had a maximum velocity per charge state of $\sim 0.5 \times 10^{9} \mathrm{~cm} / \mathrm{s}$. They also showed that resistively heating a graphite wire up to $1500{ }^{\circ} \mathrm{C}$, expelled hydrogen impurities and made possible the production of a pure carbon plasma. Irradiating this "cleaned wire" accelerated $\mathrm{C}^{6+}$ ions up to velocities of $1.25 \times 10^{9} \mathrm{~cm} / \mathrm{s}$ and $\mathrm{C}^{5+}$ ions up to $1 \times 10^{9} \mathrm{~cm} / \mathrm{s}$. The $\mathrm{C}^{6+}$ and $\mathrm{C}^{5+}$ ions appeared to self-consistently attenuate the accelerating field seen by the lower charge states in the same way that protons, when present, attenuate the field seen by the higher carbon charge states in a $\mathrm{CH}$ plasma.

An expression for the hot electron temperature was empirically determined in the 
first set of the Helios experiments to be

$$
T_{h} \simeq 14.0\left(I \lambda^{2}\right)^{1 / 3} T_{c}^{1 / 3}
$$

where $I \lambda^{2}$ is in units of $10^{16} \mathrm{~W} \mu \mathrm{m}^{2} / \mathrm{cm}^{2}$ and $T_{c}$ is the background electron temperature in $\mathrm{keV}$ at the critical density. The hot electron temperature was measured to be $23.0 \mathrm{keV}$ in the case of a $\mathrm{CH}$ plasma and a slightly higher electron temperature of $31.0 \mathrm{keV}$ in the case of a pure carbon plasma. The later observation shows that accelerating protons will reduce the electron temperature and therefore the energy coupling into the compression of a laser-fusion target.

In a large compilation of data from more than 25 laboratories and various laser systems (including $\mathrm{Kr}: \mathrm{F}$, ruby, Nd:glass, iodine, $\mathrm{CO}_{2}$ ), Gitomer et al. [31] showed that hydrogen and hydrogenous contaminants from vacuum-pump oil, water vapor and hydrocarbons are typically present on laser targets of many compositions (such as Ti, Ta and Au foils). On some targets, this hydrocarbon contamination layer may be as much as $20 \AA$ in thickness. The authors showed a strong correlation between the energy per atomic mass unit (AMU) and the hot electron temperature. The hot electron temperature was determined by measuring plasma x rays (see [31] and the references therein) and showed the electron temperature depends on the irradiance as,

$$
T_{h} \simeq a\left(I \lambda^{2}\right)^{4 / 9}
$$

where $a$ is a proportionality constant. The authors concluded, at an irradiance > $10^{16} \mathrm{~W} \mu \mathrm{m}^{2} / \mathrm{cm}^{2}$, most of the absorbed laser energy is transfered to hydrogenous 
contaminants in the form of proton kinetic energy.

\subsection{The Ponderomotive Potential Revisited}

As laser intensities increased, simulations [23] and later experiments [32, 33, 34] began to show that ions could be accelerated up to energies of several $\mathrm{MeV}$ due to the large radiation pressure exerted on the plasma. At this point, it is beneficial to go through the derivation of the radiation pressure to ensure accurate use of the term.

The energy flux density of an EM wave is described by Poynting's vector as,

$$
\vec{S}=\frac{1}{\mu_{o}}(\vec{E} \times \vec{B}) .
$$

For the case of a mono-chromatic plane wave in the $\hat{z}$ direction,

$$
\vec{E}=E_{o} \cos \left(k z-\omega_{o} t\right) \text { and } \vec{B}=\frac{1}{c} \vec{E} \text { (in vacuum). }
$$

Plugging the E and B fields into Poynting's vector yields,

$$
\vec{S}=c \epsilon_{o} E_{o}^{2} \cos ^{2}\left(k z-\omega_{o} t\right) \hat{z} .
$$

Next, we define the intensity as the time average of the energy density flux,

$$
I \equiv\langle\vec{S}\rangle=\frac{1}{2} c \epsilon_{o} E_{o}^{2} \hat{z}
$$

And finally, the radiation pressure is merely the energy density given by,

$$
P_{\text {rad }}=\frac{I}{c}=\frac{1}{2} \epsilon_{o} E_{o}^{2} \hat{z} .
$$




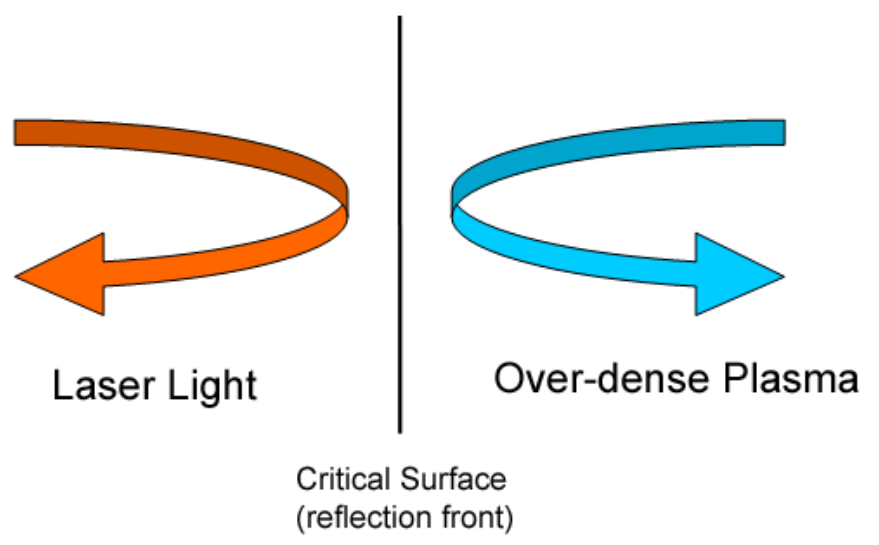

Figure 3.1: At the plasma critical surface, the ponderomotive potential is equivalent to the classical radiation pressure. This pressure can be hundreds of Mbar for intensities $>10^{19} \mathrm{~W} / \mathrm{cm}^{2}$.

We can see from Eq. (3.18) that ultra-high intensities can produce radiation pressures hundreds of megabars in magnitude. For example, an intensity of $I=10^{19} \mathrm{~W} / \mathrm{cm}^{2}$, corresponds to a radiation pressure of $P_{\text {rad }} \approx 300$ Mbar.

If the energy density of the EM wave greatly exceeds the kinetic energy of the plasma, the critical surface can be "pushed" inward, launching an ion shock wave. We can solve for the velocity of the ion wave by balancing the radiation pressure with the ion momentum flux in the frame of the reflection surface:

$$
P_{\text {rad }}=n M_{i} v_{i}^{2}
$$

At this reflection surface, the plasma frequency is equal to the frequency of the laser light $\left(\omega_{p e}=\omega_{o}\right)$ and we see that the ponderomotive potential reduces to the radiation pressure from Eq. (3.18):

$$
U_{p}=\frac{\omega_{p e}^{2}}{\omega_{o}^{2}} \frac{1}{2} \epsilon_{o} E_{o}^{2}=\frac{1}{2} \epsilon_{o} E_{o}^{2}=P_{r a d} .
$$


This allows us to set the ion momentum flux, equal to the ponderomotive potential,

$$
v_{i}^{2}=\frac{U_{p}}{n M_{i}},
$$

and solve for the ion velocity in more familiar terms [23]:

$$
\frac{v_{i}}{c}=\sqrt{\frac{n_{c}}{2 n_{e}} \frac{Z m_{e}}{M_{i}} \frac{I \lambda_{\mu}^{2}}{1.37 \times 10^{18}}} .
$$

Solving the above equation for typical values of, say, $I=2 \times 10^{19} \mathrm{~W} / \mathrm{cm}^{2}$, ion velocities of $v_{i} \approx 0.063 c$ are possible. We can calculate the energy for a proton by substituting this velocity and the proton mass $\left(938.2700 \mathrm{MeV} / c^{2}\right)$ into the classical energy equation:

$$
E_{\text {proton }}=\frac{1}{2} M_{i} v_{i}^{2}=\frac{1}{2}\left(938.27 \frac{\mathrm{MeV}}{c^{2}}\right)(0.063 c)^{2} \approx 1.86 \mathrm{MeV} .
$$

When ion acceleration up to $\mathrm{MeV}$ energies was first observed in laser-plasma experiments with laser intensities $I>10^{19} \mathrm{~W} / \mathrm{cm}^{2}$, it was thought to be exclusively the result of ponderomotive acceleration. In the next several sections we review several recent experiments and present evidence of an additional acceleration mechanism, a strong electrostatic sheath on the rear surface of the laser target, that may be the dominant acceleration mechanism within specific experimental parameters.

\subsection{Observation of High Energy Protons}

In the late 1990's and the year 2000, research groups at various institutions observed protons with energies of several $\mathrm{MeV}$ from the laser irradiation of solid foil- 
targets. No sooner was proton acceleration observed, than was the responsible mechanism debated. In the sections that follow, we present several mechanisms of ion acceleration with respect to various experimental parameters.

\subsubsection{The Michigan Experiments}

One of the first groups to observe ion acceleration from CPA laser-plasma interactions was Maksimchuk et al. at the Center for Ultrafast Optical Science at the University of Michigan [32]. Experiments were performed with a 10 TW hybrid Ti:sapphire/Nd:glass laser capable of generating laser pulses of $4 \mathrm{~J}$ in $400 \mathrm{fs}$. At the system's fundamental wavelength of $\lambda=1.053 \mu \mathrm{m}$, the contrast of the main pulse intensity to the prepulse was $10^{5}: 1$. The contrast was improved to $10^{7}: 1$ by using a $4 \mathrm{~mm}$ KDP crystal to double the frequency of the laser pulse. Because frequency doubling is a non-linear effect, the high-intensity main pulse is more efficiently doubled to a wavelength of $\lambda=0.532 \mu \mathrm{m}$ than the lower-intensity prepulse, making the later easier to separate - or remove entirely - through the use of wavelength specific optics. The maximum focused intensity was $3 \times 10^{18} \mathrm{~W} / \mathrm{cm}^{2}$ at the doubled wavelength.

Energetic protons were observed up to energies of $1.5 \mathrm{MeV}$ from the laser irradiation of thin $(\approx 1.5 \mu \mathrm{m})$ aluminum and Mylar foils. Using a combination of CR-39 (a nuclear track detector sensitive only to ions with energies greater than $100 \mathrm{keV} /$ nucleon) and Mylar filters serving as attenuators, the authors measured a proton spectrum with a resolution of about $100 \mathrm{keV}$. The proton spectrum showed 
an exponential decay from $400 \mathrm{keV}$ to $1 \mathrm{MeV}$ with a temperature of $230 \mathrm{keV}$. From $1 \mathrm{MeV}$ to $1.5 \mathrm{MeV}$ there existed a plateau, ending sharply at the cutoff energy of 1.5 MeV. This plateau region just before a sharp cutoff is a characteristic feature of electrostatic acceleration.

To determine from which side of the target ions were accelerated, the authors measured nuclear activation of ${ }^{10} \mathrm{~B}$ (from the reaction ${ }^{10} \mathrm{~B}(d, n){ }^{11} \mathrm{C}$ ) by laser-accelerated deuterons. A sample of boron was placed $1 \mathrm{~cm}$ behind the target which was a $6 \mu \mathrm{m}$ Mylar foil with a thin layer of deuterated plastic on the front surface. When the target was laser irradiated in this arrangement, they measured $10^{5}$ atoms of the positronactive isotope ${ }^{11} \mathrm{C}$. When a the layer of deuterated plastic was added to the back side of the foil, no activation was measured. In their own words, "These results indicate unequivocally that for the condition of our experiment ions are accelerated from the front side of the thin foil."

The authors showed that proton energies depend predominately on the plasma scale-length of the front surface, and for a high-contrast system can be explained by "vacuum heating" at the sharp interface due to the Brunel effect of the $\vec{v} \times \vec{B}$ force. High energy electrons generated on the front surface are driven into the target, producing a strong electrostatic field, accelerating ions in the forward direction. Protons that originate from hydrogenous contaminants on the front surface, are accelerated through the thin film target and emerge through the rear surface as a high energy beam with an angular divergence of $40^{\circ} \pm 10^{\circ}$. 
Although, these experiments show that for certain experimental parameters deuterons were accelerated from the front surface of the target, they do not conclusively show that acceleration of ions from the back surface is not possible under different experimental parameters, for several reasons. Firstly, the targets used in the Michigan experiments were so thin, the plasma skin-depth could only be several wavelengths of the laser light. It is difficult to imagine the back surface of the target could remain sufficiently cold and unperturbed long enough for a posterior, strong gradient electrostatic sheath to develop. Secondly, in the case where the deuterated plastic was added to the back surface, there was no effort to clean the target of hydrogenous contaminants. Therefore, even if an electrostatic sheath did exist on the back surface, protons from contaminants would have been preferentially accelerated, attenuating the electric field necessary to accelerate deuterons. Such an experiment could only be performed with a method of removing the hydrogenous contaminants from the target without effecting the deuterium dopant one wishes to accelerate.

\subsubsection{The RAL Experiments}

A multi-institutional collaboration at the Rutherford Appleton Laboratory (RAL) has also contributed extensively to laser-ion acceleration research. Clark et al. describe high energy proton beams with measured energies up to $\approx 18 \mathrm{MeV}$ [33]. The RAL short-pulse system, is capable of $\lambda=1.053 \mu \mathrm{m}, 50 \mathrm{~J}$ laser pulses with duration of $0.9-1.2 \mathrm{ps}$, producing focused intensities up to $I=5 \times 10^{19} \mathrm{~W} / \mathrm{cm}^{2}$. They irradiated 
aluminum targets of $125 \mu \mathrm{m}$ thickness (much thicker than the Michigan experiments) at a $45^{\circ}$ angle of incidence to the front surface of the target. To detect ions, they used a combination of CR-39 and radiochromic film (RCF). RCF is composed of an organic dye sandwiched between two pieces of a polymer (typically nylon). The dye, which is normally transparent, turns blue when exposed to ionizing radiation. The properties of RCF and how it can be used to measure radiation dose and therefore integrated yield of charged particle acceleration will be discussed extensively in the next chapter.

Placing alternating layers of CR-39 and RCF (both $\approx 2 \times 2$ in $^{2}$ in area) several inches behind the laser target, the authors observed an ion beam with a cone halfangle of $30^{\circ}$, with a center hot spot corresponding to proton energy $\approx 18 \mathrm{MeV}$. They also observed what appeared to be an "ion ring" structure on successive layers of CR39. This structure, apparent up to energies of $\approx 10 \mathrm{MeV}$, led them to postulate the existence of magnetic fields $>30 \mathrm{MG}$, which deflected ions into a ring-like pattern.

Strong magnetic fields can certainly be generated from the electron current of high-energy electrons in the laser plasma. Such fields would tend to focus electron beams, but defocus beams of positively charged ions. The RAL group contends that the energy of the emitted protons and the angle at which they are emitted is caused by an azimuthal magnetic field established by the high-energy electron current in the first few picoseconds of the laser-plasma interaction. While the authors do admit that the protons must come from either hydrogenous contaminants on either the front or 
back surface of the target, they claim it is "highly unlikely" that the protons originate from the back surface:

A simple estimate shows that unrealistic field strengths of $>1 \times 10^{9} \mathrm{G}$ are required to achieve the observed proton deflections over a distance of a few microns (which is the size of the rear surface plasma). It is more probable that the energetic protons are generated at the front surface and are subsequently transported through the target. [original parenthesis]

The author's conclusions do not disallow the existence of a back-surface electrostatic sheath. Its existence is simply considered improbable due to the high magnetic field that would be required to produce the observed ring structure.

\subsubsection{The Petawatt Experiments}

The Petawatt laser (introduced in the previous chapter, c.f. Hatchett et al. [34] and Snavely et al. [35]) at the Lawrence Livermore National Laboratory (LLNL) was capable of several hundreds of joules of $1 \mu \mathrm{m}$ laser light in $0.5-5 \mathrm{ps}$, which produced an irradiance on target in excess of $3 \times 10^{20} \mathrm{~W} \mu \mathrm{m}^{2} / \mathrm{cm}^{2}$. Using the large Nd:glass amplifiers allowed the LLNL petawatt to generate high intensities, but resulted in poor contrast with a 2 ns prepulse at $10^{-4}$ of the main pulse intensity. This resulted in a preplasma with an on-axis electron density of $3 \times 10^{-19} \mathrm{~cm}^{-3}$ in a plane $70 \mu \mathrm{m}$ in front of a flat target, with an exponential fall off to lower densities having a scale length of $40 \mu \mathrm{m}$. A powerful prepulse such as this demands target thicknesses greater than, at least, tens of microns to ensure the target is not blown away before the arrival of the main pulse. 
The highest proton yield as well as the highest proton energies to date were observed at this facility. Irradiating a range of $\mathrm{Au}$ and $\mathrm{CH}$ targets with thicknesses from 20 to $120 \mu \mathrm{m}$ at angles of incidence both normal and $45^{\circ}$ always produced an intense beam of protons perpendicular to the rear surface of the target. Using a detector stack of alternating titanium foils and RCF placed behind the laser target, a rough proton spectrum could be calculated. The maximum penetration of multiple layers of the detector pack showed the existence of protons with energies $>40 \mathrm{MeV}$, and the attenuation with thickness gave a slope temperature of $6 \mathrm{MeV}$. An absolute proton yield was measured by nuclear activation of titanium. The ${ }^{48} \mathrm{Ti}(p, n)^{48} \mathrm{~V}$ reaction has a threshold of $5 \mathrm{MeV}$ and a peak cross-section of $\approx 500 \mathrm{mb}$ at $12 \mathrm{MeV}$. The total number of protons above threshold was determined to be $\sim 3 \times 10^{13}$, which represents $30 \mathrm{~J}$ or $7 \%$ of the laser pulse energy.

In an effort to specifically determine whether the protons observed by the detectors originated from the front or back surface of the target, a $2 \mathrm{~mm}$ wide $30^{\circ}$ wedge target of $\mathrm{CH}$ was irradiated. Strong evidence that the accelerating field existed at the rear surface was seen in two distinct proton beams corresponding and normal to the major and minor "rear" surfaces of the target as shown in Fig. 3.2.

If protons originated on the front surface and propagated through the target, there would be a visible spot in the middle of the film. Instead, the only indication of protons is recorded on sections of the film that were perpendicular to the wedge's "rear" surfaces. The authors also explain that, in the case of the Petawatt laser, 


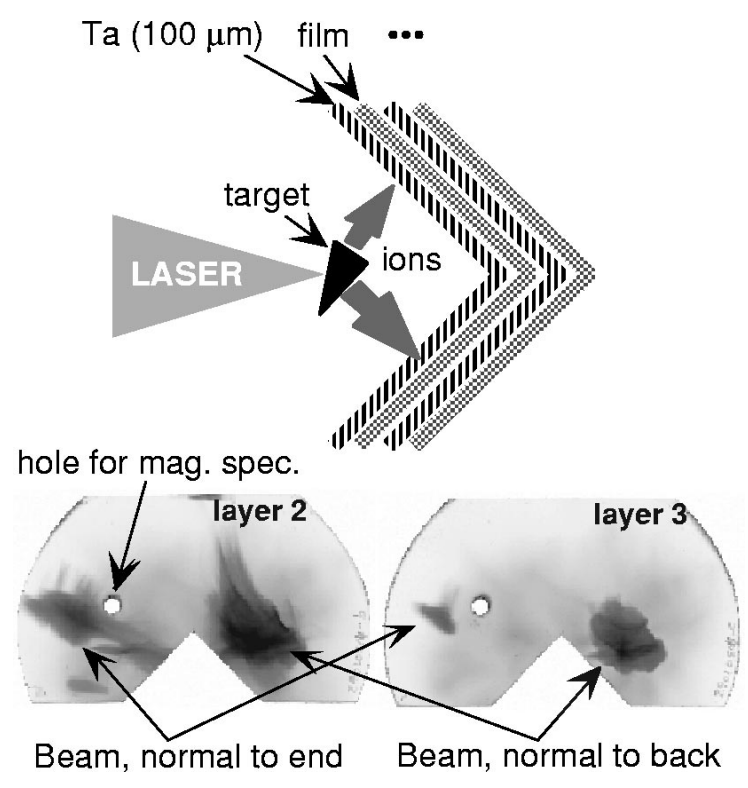

Figure 3.2: Wedge target schematic and RCF images via 200 and $300 \mu \mathrm{m}$ Ta (protons $>14$ and $>17 \mathrm{MeV}$ ). The image shows two protons beams, the larger from the major face and the smaller from the minor face of the wedge. Used with permission from S.P. Hatchett $[34]$.

where contrast was low and prepulse intensity was high. Contaminants on the front surface would be blown away before the arrival of the main pulse. Not to mention that an area much larger than the $10-\mu \mathrm{m}$ diameter focal spot size would be needed to supply the observed number of protons. In the next section, a theoretical model of rear-surface ion acceleration is presented and discussed.

\subsection{Target Normal Sheath Acceleration}

The acceleration mechanism, now referred to as target normal sheath acceleration (TNSA), is very similar to the electrostatic model described in Section 3.1.1 except the 
scaling for $T_{\text {hot }}$ is now given by the ponderomotive potential instead of by resonance absorption.

The hot-electron energy spectrum in a laser plasma has been shown to resemble a relativistic Maxwellian distribution with an effective temperature

$$
k_{B} T_{h o t} \cong m c^{2}\left(1+\frac{U_{p}}{m c^{2}}\right)^{1 / 2}
$$

which scales with the ponderomotive potential of the laser [36]. This model, described below, was presented initially by Hatchett et al. [34] and discussed in further detail by Wilks et al. [37].

Following the Petawatt experimental parameters, we assume the laser prepulse creates a plasma on the front surface of the target that expands spherically into vacuum. The main pulse interacts with this preplasma when it arrives $\sim 2$ ns later in time, and generates a large number of hot electrons near the region of the critical surface. Only a very small fraction of these electrons can escape the target before the rest are trapped by the resulting Coulomb potential. Those contained by the target's potential will bounce back and forth through the target while drifting transversely. As the electrons relax to a Boltzmann equilibrium, they will establish a sheath on the back surface with a scale length initially equal to the Debye length of the hot electrons,

$$
\ell_{o} \equiv\left(\frac{k_{B} T_{h o t}}{4 \pi e^{2} n_{e, h o t}}\right)^{1 / 2}=2.4 \mu m\left(\frac{k_{B} T_{h o t}}{1 \mathrm{MeV}}\right)^{1 / 2}\left(\frac{n_{e, h o t}}{10^{19} \mathrm{~cm}^{-3}}\right)^{-1 / 2}
$$

We can see from the above equation that the hot-electron Debye length is of order sev- 
eral microns for the electron temperatures and densities relevant to our experimental parameters.

This combination of relativistic electron temperature and high hot-electron density will result in a very strong electrostatic sheath field,

$$
\vec{E} \approx \frac{k_{B} T_{h o t}}{e \ell_{o}}=\frac{\text { megavolts }}{\text { microns }} .
$$

This electric field is sufficiently strong to field ionize the contaminants on the back surface of the target and accelerate them to high energies over the short distance that the sheath extends. Such an intense gradient in field strength can only be achieved with an ultra-short pulse where a large number of electrons are created in a time period much shorter than the target disassembly time. The hot electrons establish a very short scale-length sheath plasma before the shock wave (traveling at the sound speed) launched on the front surface can disrupt the back surface and elongate the plasma scale length.

As the plasma expands on the rear surface, the ions carry electrons with them in a region of quasi-neutrality, allowing for the production of beam currents above the vacuum Alven limit. Ahead of the expanding ion front, where the local hot electron Debye length is greater than the local ion scale length the hot electron sheath exists. As the electrons lose energy by ranging out, adiabatic cooling or by accelerating ions the electron Debye length will become equal to the ion scale length. When this occurs, there is no longer a strong charge separation, therefore no electrostatic field, and the acceleration process stops. Particle-in-cell (PIC) models have shown that even though 


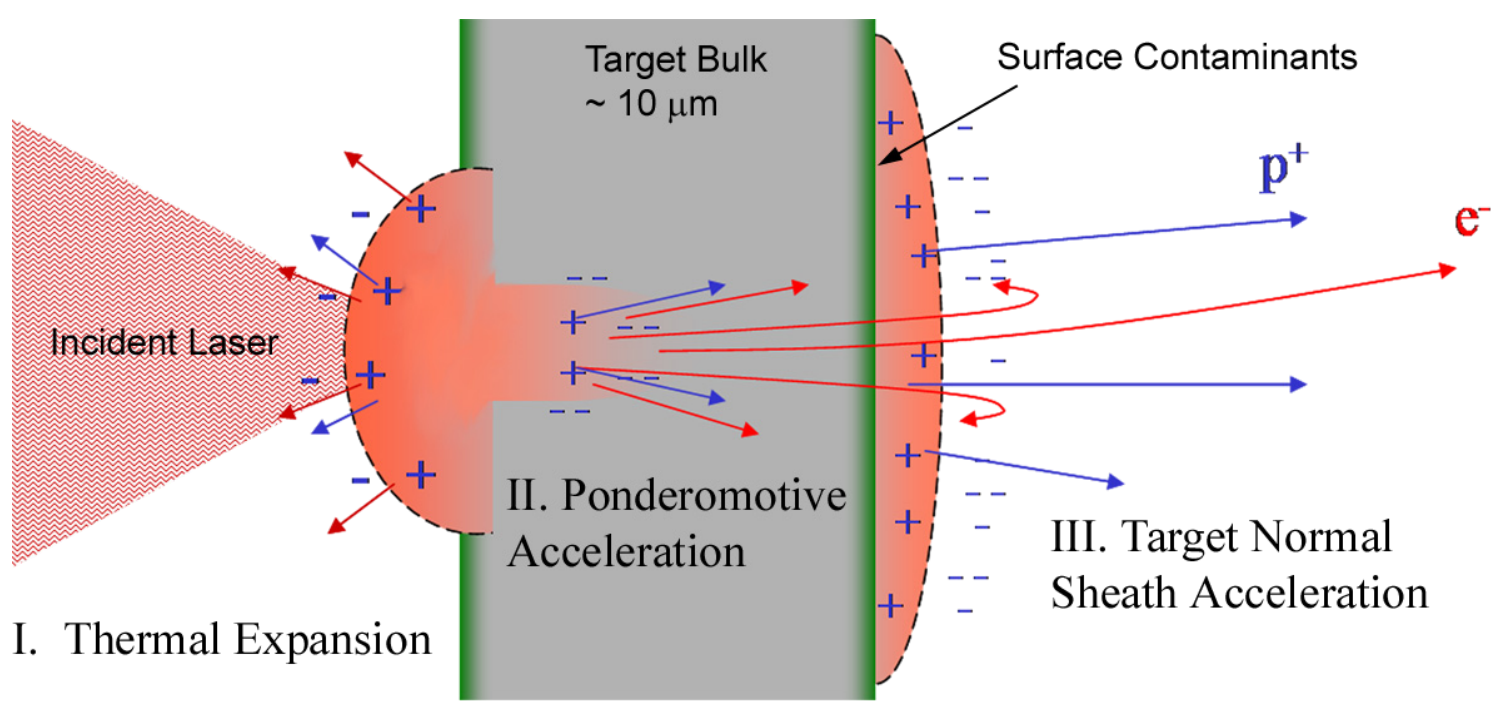

Figure 3.3: Schematic of three main processes governing ion acceleration from a laser target: I. Thermal Expansion $E_{i o n} \sim$ sub-MeV. II. Ponderomotive Acceleration $E_{\text {ion }}=1-3 \mathrm{MeV}$, on the order of the laser ponderomotive potential. III. Target Normal Sheath Acceleration $E_{\text {ion }}>10 \mathrm{MeV}$

the acceleration process lasts for less than 1 ps [38], the accelerating gradients are sufficient for ions to gain energies of tens of $\mathrm{MeV}$.

In conclusion, it should be stated that ions can be accelerated by several different mechanisms, as shown in Fig. 3.3. The thermal expansion of the plasma will accelerate ions up to energies of tens of $\mathrm{keV}$. The ponderomotive potential of the laser pulse can accelerate ions from the front surface of the laser target up to several $\mathrm{MeV}$. The TNSA model describes a back-surface electrostatic sheath capable of accelerating protons and heavier ions up to tens or hundreds of $\mathrm{MeV}$. A theoretical review of the various acceleration mechanisms discussed above is presented by Pukhov in Ref. [38]. 


\section{Chapter 4}

\section{Particle Detection: Instruments}

\section{and Diagnostics}

Originally, laser-plasma experiments were diagnosed with film based detectors such as x-ray pinhole cameras, x-ray microscopes, and spectrometers. As chargecoupled-devices (CCDs) developed higher dynamic ranges and quantum efficiencies, film based detectors were gradually phased out of many large scale laser-plasma experiments. CCDs offered the benefit of instantaneous, on-line analysis without the delays and inconsistencies associated with the processing of film. However, in the regime of ultra-short, ultra-intense laser-plasma interactions with solid targets, the emitted gamma radiation due to bremsstrahlung conversion of hot electrons in the target material is sufficiently intense to damage most CCDs and other electronic diagnostics. Therefore, a good choice for diagnosing laser-accelerated ions are instruments 
that are both static and film based.

In this chapter, we describe the primary diagnostics used in experiments discussed in the following chapters: radiochromic film and nuclear activation of metallic foils. Radiochromic film recorded 2-D spatial information about the emitted proton beam as well as total proton yields, and a gross energy spectrum. Nuclear activation of thin, metallic foils was useful in determining the total yields of protons (in the case of titanium activation) and neutrons (in the case of gold activation).

\subsection{Radiochromic Film}

Radiochromic film (RCF) is a self-developing, radiation-sensitive film composed of a transparent material that is coated with an organic dye. Upon exposure to ionizing radiation, the film turns blue in color hence the term "radio"- "chromic". This color change is a function of exposure; higher doses of radiation result in higher optical densities of the film. The sensitivity of RCF is related to deposited energy, and is low with respect to gamma rays and minimum-ionizing electrons that pass through the film without losing much energy. However, with respect to protons and heavier ions that often reach their Bragg peak, depositing all of their energy in the film, RCF has a high sensitivity.

The particular brand of RCF used in the experiments presented here was GAFCHROMIC ${ }^{\circledR}$ dosimetry film manufactured by International Specialty Products (ISP) [39]. Three distinct varieties of RCF were used in the experiments, each with their own strengths 


\begin{tabular}{|c|c|c|c|c|c|}
\hline \multicolumn{2}{|l|}{ MD-55 } & \multicolumn{2}{|c|}{ HD-810 } & \multicolumn{2}{|c|}{ HS } \\
\hline Layer & Thickness $\mu \mathrm{m}$ & Layer & Thickness $\mu \mathrm{m}$ & Layer & Thickness $\mu \mathrm{m}$ \\
\hline Polyester Base & 66 & Surface & 0.75 & Polyester & 96.5 \\
\hline Sensitive Layer & 16 & Active Layer & 6.5 & Active Layer & 40 \\
\hline Pressure-sensitive Adhesive & 25.4 & Polyester & 96.5 & Polyester & 96.5 \\
\hline Polyester Base & 25.4 & & & & \\
\hline Pressure-sensitive Adhesive & 25.4 & & & & \\
\hline Sensitive Layer & 16 & & & & \\
\hline Polyester Base & 66 & & & & \\
\hline Total Thickness & 240.2 & Total Thickness & 103.75 & Total Thickness & 233 \\
\hline $\begin{array}{l}\text { Table 4.1: } \\
\text { GAFCHROMIC } \\
\text { http://www.ispcorp.cor } \\
\text { hto }\end{array}$ & $\begin{array}{l}\text { descri } \\
\text { netry film } \\
\text { roducts } / \text { do }\end{array}$ & $\begin{array}{l}\text { ion of } \\
\text { Informa } \\
\text { metry. }\end{array}$ & $\begin{array}{l}\text { [nternational } \\
\text { ion taken }\end{array}$ & $\begin{array}{l}\text { Specialty } \\
\text { from compan }\end{array}$ & $\begin{array}{l}\text { Products' } \\
y \quad \text { website }\end{array}$ \\
\hline
\end{tabular}

and shortcomings. MD-55, for example, is a high-sensitivity film with two sensitive layers each $15-\mu \mathrm{m}$ thick on a polyester base of $67 \mu \mathrm{m}[40]$. The high sensitivity of this film makes it a good choice in measuring low signals, but the thick layers of polyester prevent the detection of protons below $\sim 6 \mathrm{MeV}$. HD-810 has no initial polyester layer and is capable of detecting very low energy particles, however, it is less sensitive due to its thinner active dye layer. A detailed description of each film composition is given in Table 4.1. For the detection of highly ionizing radiation, RCF is more convenient than x-ray film since, unlike x-ray film, it is not strongly sensitive to room light and requires no processing. The film is purchased in 5 in. $\times 5$ in. sheets and can easily be cut into sizes appropriate for experiments.

Proton yields at specific proton energies can be measured by placing a stack of 


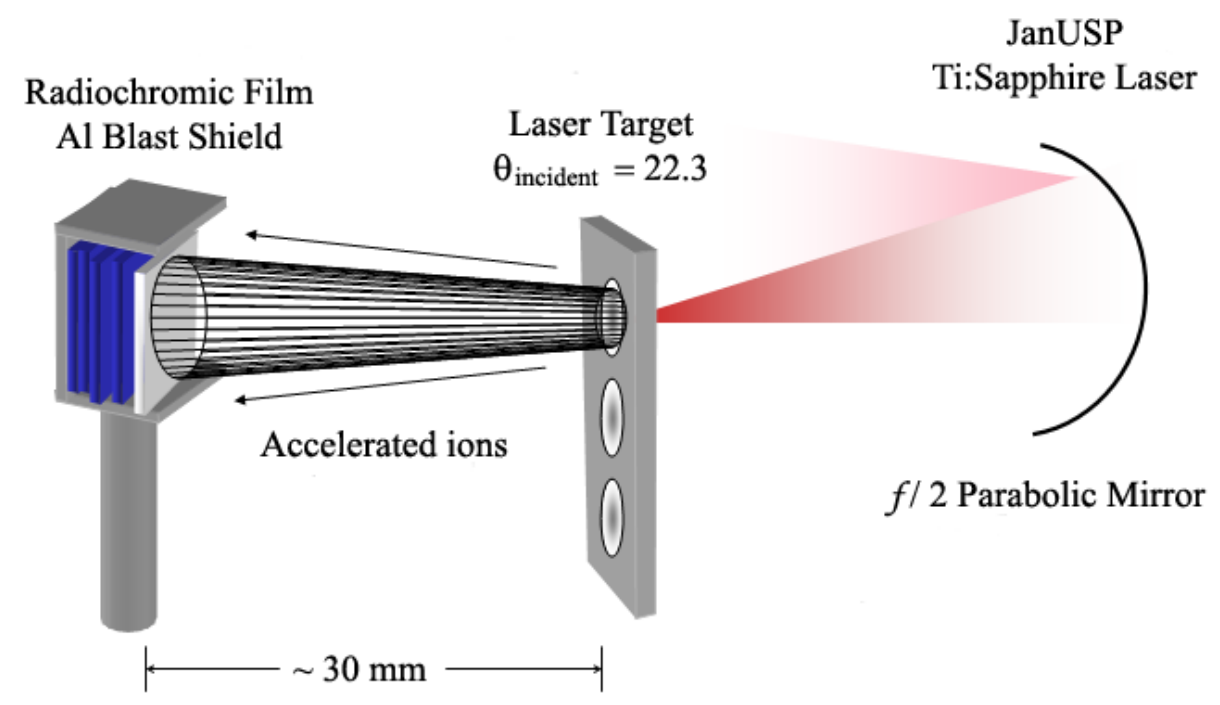

Figure 4.1: Experimental setup for laser-accelerated ions using RCF as the principle diagnostic. An aluminum blast shield protects the film pack from target blow off and debris. The first piece of film (layer 1), directly behind the blast shield, is often saturated.

$\mathrm{RCF}$ behind the laser target, normal to the back surface in direct line with the generated ion beam as shown in Fig. 4.1. The optical density of each individual piece of RCF can then be measured, and the specific dose from energetic protons can be calculated.

The deposited dose as a function of optical density (O.D.) for MD-55 was determined by irradiating the film with a cobalt-60 source of known activity. The deposited dose was found to depend on O.D. as

$$
\begin{aligned}
& \operatorname{Dose}(D)= \\
& \quad 1.12 \times 10^{-10.8379 \times D^{0.1}+30.517 \times D^{0.5}-30.86 \times D^{0.9}+13.668 \times D^{1.3}-2.09 \times D^{1.7}}
\end{aligned}
$$

where $D$ is the optical density of the film. From Fig. 4.2, which shows a plot of 


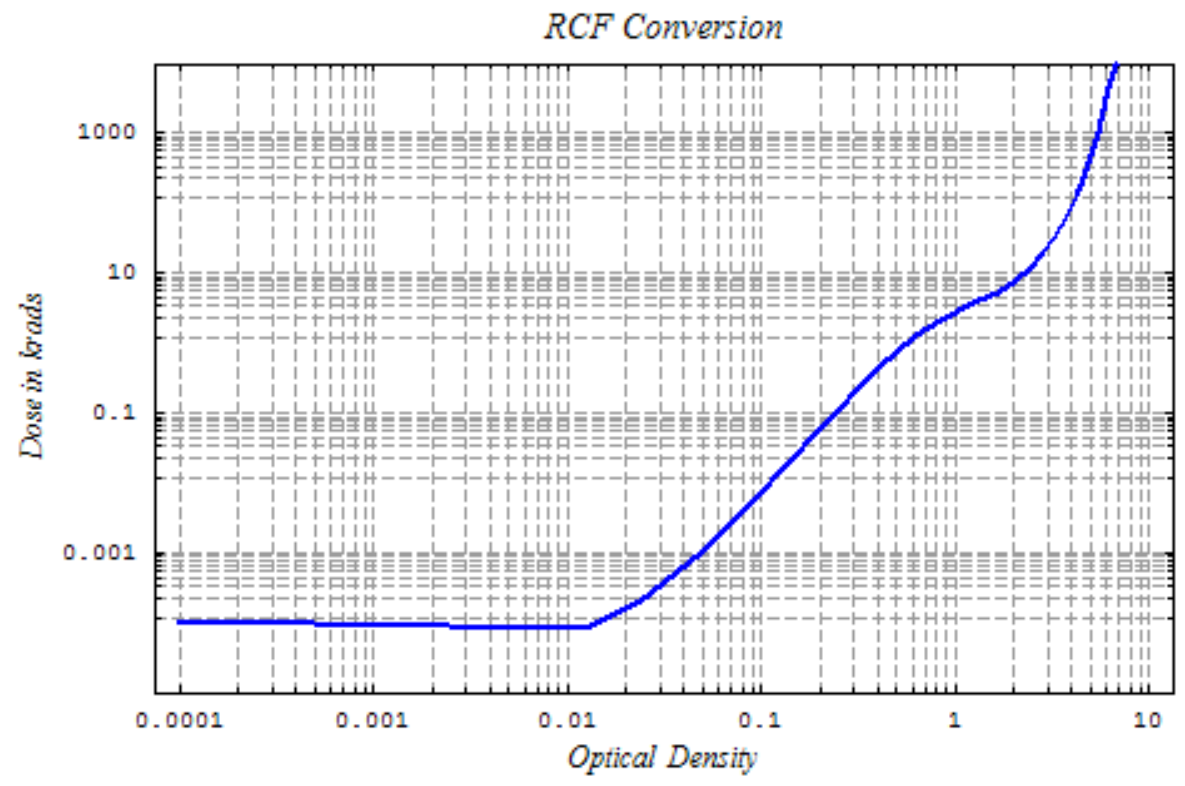

Figure 4.2: Deposited dose in krad as a function of optical density for RCF.

Eq.(4.1) over the typical range, we can see the function is highly nonlinear. This nonlinearity increases the signal to noise ratio and decreases (but does not eliminate) the need for background subtraction. The accuracy of the deposited dose function was confirmed by cross calibration of the film with nuclear activation techniques as described in the next section.

The film was digitized with a Perkin-Elmer Photometric Data System (PDS) using a $22 \mu \mathrm{m} \times 22 \mu \mathrm{m}$ square aperture. Images were stored as unsigned 12-bit data files with a maximum pixel value of 4096 . The density factor was set at 0.00125 . Therefore the optical density, defined as

$$
\text { O.D. }=\text { pixel value } \times 0.00125
$$



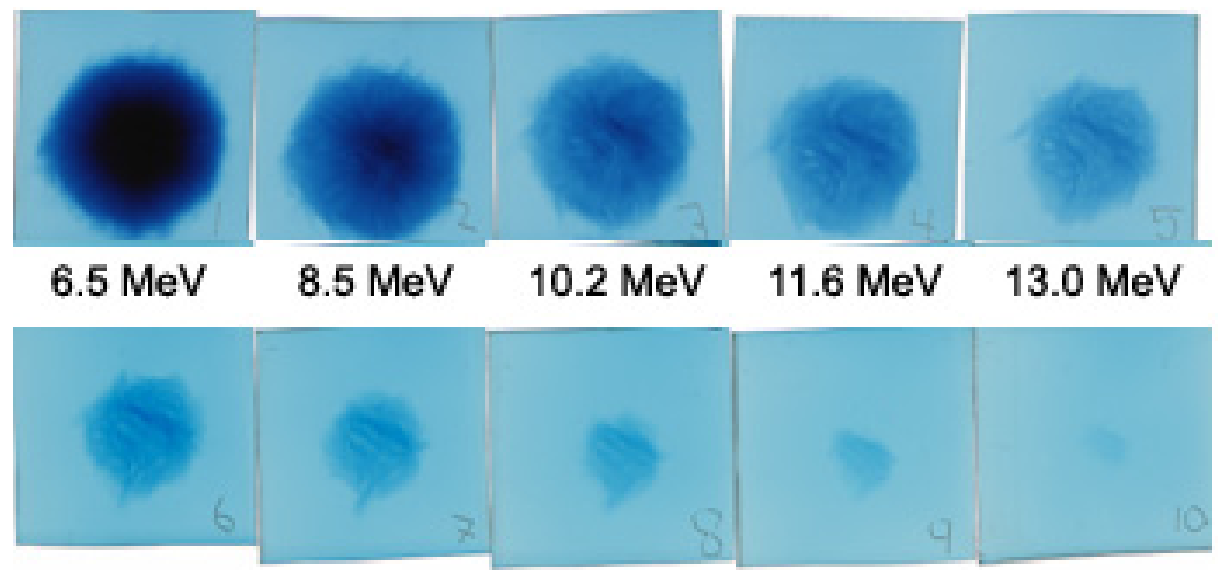

\section{$\begin{array}{llll}14.2 \mathrm{MeV} & 16.4 \mathrm{MeV} \quad 18.4 \mathrm{MeV} \quad 19.3 \mathrm{MeV} \quad 20.0 \mathrm{MeV}\end{array}$}

Figure 4.3: RCF from JanUSP shot\# 10011605. Laser target was $25-\mu \mathrm{m}$ thick Pd; the laser energy was $10.7 \mathrm{~J}$. Protons evident up to the tenth layer of film show the existence of proton energies $>20 \mathrm{MeV}$.

gives a maximum of $O \cdot D .=5.12$. Since the first layer of the RCF pack (layer 1 ) is often saturated, the majority of information about the proton beam is gained from examining layers 2 and deeper.

An example of exposed RCF is shown in Fig. 4.3. Each piece of RCF is approximately 1-in. square, and the film stack was approximately $20 \mathrm{~mm}$ behind the laser target. The absorbed dose peaks in the center and decreases radially outward along the beam profile. The diameter of the beam progressively decreases with each increasing layer of RCF due to protons ranging out in each layer of the film. In this particular shot, protons are apparent up to the tenth layer of RCF, corresponding to a maximum proton energy of $>20 \mathrm{MeV}$.

To model the observed proton spectrum, we developed a code that incorporates 


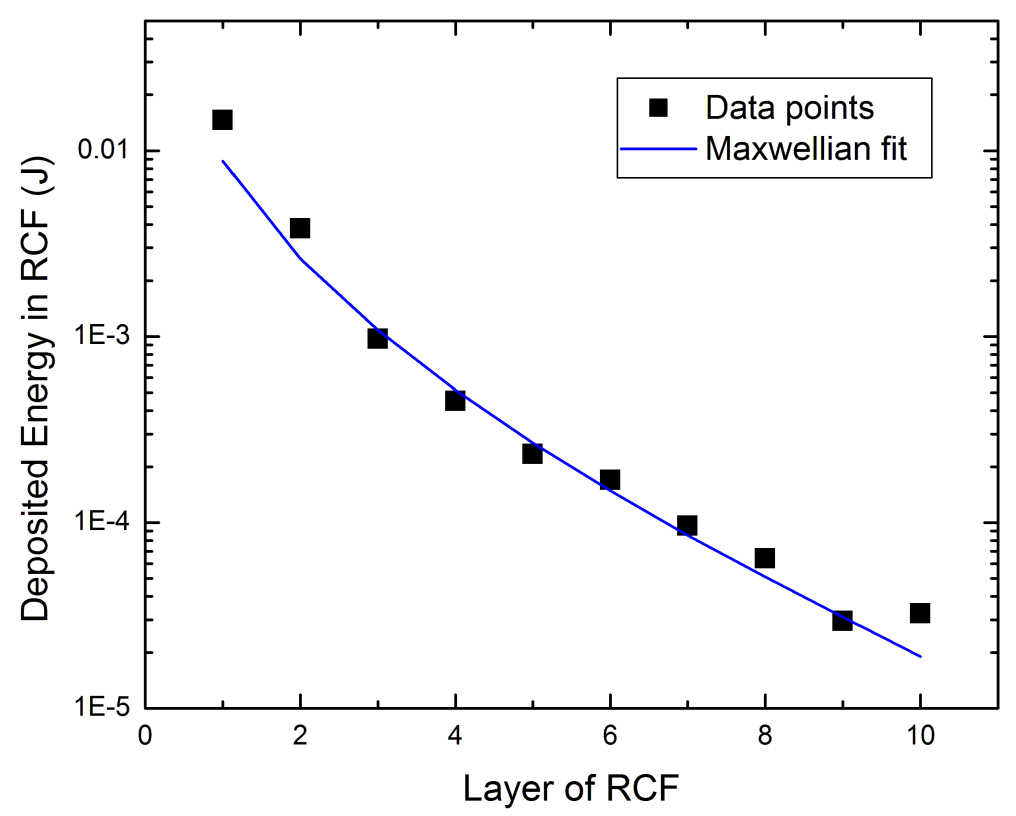

Figure 4.4: Analysis of JanUSP shot\# 10011605. Laser target was $25-\mu \mathrm{m}$ thick Pd, and the laser energy was $10.7 \mathrm{~J}$. Proton number $\left(N_{o}\right)$ and temperature $(k T)$ were found to be $3 \times 10^{11}$ and $2.1 \mathrm{MeV}$ respectively.

the same combination of empirical and analytical energy deposition theory as that of SRIM (c.f. [41, 42, 43] and the ref. therein), a group of programs that calculate the stopping and range of ions into matter using a quantum mechanical treatment of ion-atom collisions. The code we developed is written in a combination of Java and Mathematica to achieve a high computational speed while allowing ease of data manipulation and real-time plotting of results, without the necessity of recompiling the code every time the input changes. ${ }^{1}$ The code divides the film pack into $1-\mu \mathrm{m}$ layers and calculates the deposited energy of an incident proton as it propagates

\footnotetext{
${ }^{1}$ The source for our code is listed in Appendix B.
} 
through successive layers. Each incident proton from energies of zero up to $20 \mathrm{MeV}$ is normalized to a Maxwellian distribution of the form

$$
N(E)=N_{o} \frac{2}{\sqrt{\pi}(k T)^{3 / 2}} \sqrt{E} e^{-E / k T},
$$

where $N_{o}$ represents total proton number and $k T$ is the temperature of the distribution in $\mathrm{MeV}$.

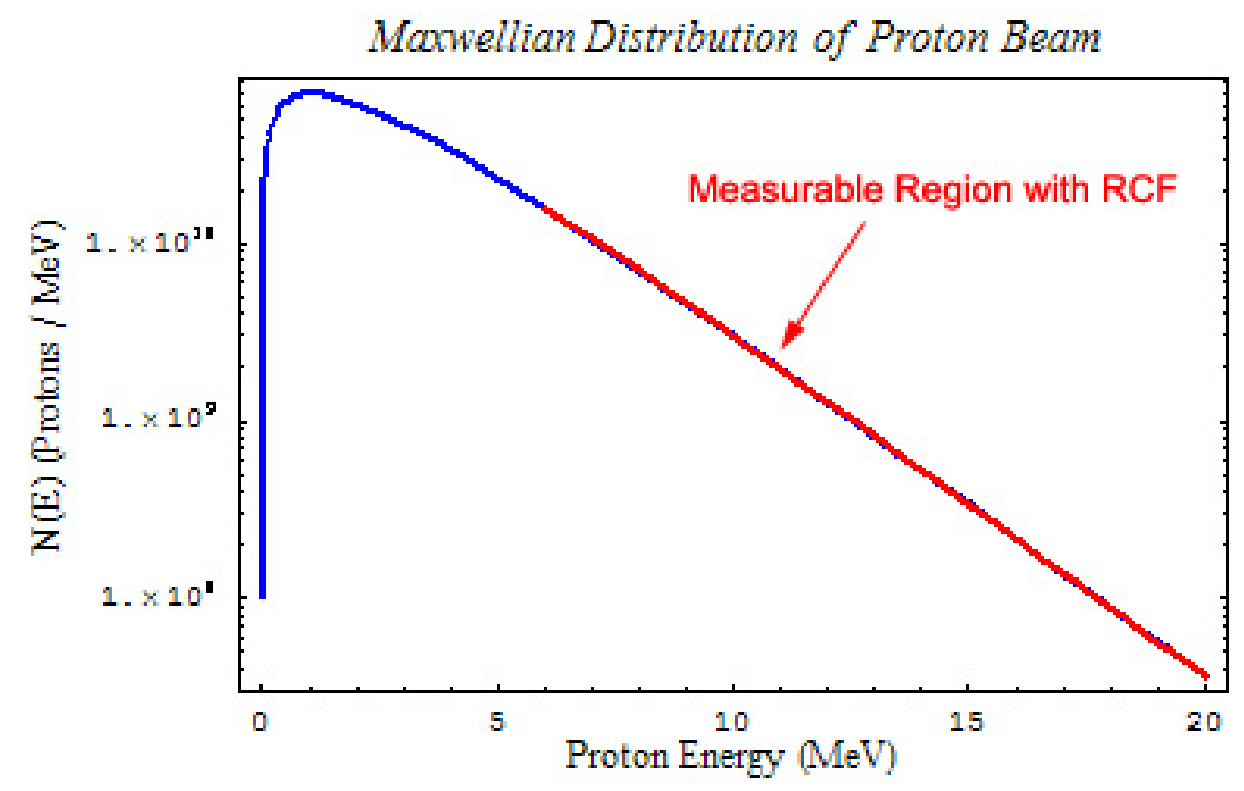

Figure 4.5: Maxwellian distribution of proton beam, where proton number $\left(N_{o}\right)$ and temperature $(k T)$ are $3 \times 10^{11}$ and $2.1 \mathrm{MeV}$ respectively.

Using this method, the energy deposited in each active dye layer of RCF is plotted with the measured dose of the experimental data obtained from the optical density. The proton number $\left(N_{o}\right)$ and temperature $(k T)$ are then adjusted interactively to achieve the best fit with the data. For illustration, the analysis of the data shown in Fig. 4.3 is presented in Fig. 4.4. For this particular shot, the proton number and 
temperature were found to be $3 \times 10^{11}$ and $2.1 \mathrm{MeV}$ respectively. A Maxwellian distribution with this yield and temperature is shown in Fig. 4.5. As shown in the figure, the film only measures the portion of the distribution above $6 \mathrm{MeV}$. For this particular shot, protons $>6 \mathrm{MeV}$ represent $\approx 13 \%$ of the total distribution. Although this is sufficient to fit a Maxwellian, it is also possible to fit an exponential function to the same data points. A Maxwellian fit was selected because the boundary condition $N(0)=0$ seems more physical than the associated boundary condition for an exponential. To date, no one has been able to accurately measure the low-energy end of the observed proton spectrum.

\subsection{Nuclear Activation Counting}

\subsubsection{Charged Particle Detection with Titanium}

Charged particle detection has played an important role in diagnosing laseraccelerated protons and deuterons. Snavely et al. used nuclear activation of titanium to measure proton yields in the first Petawatt experiments at LLNL [35]. Titanium is an attractive choice for proton detection since the ${ }^{48} \mathrm{Ti}(p, n)^{48} \mathrm{~V}$ reaction has a threshold of $5 \mathrm{MeV}$ and a peak of $\sim 500$ mbarn at $12 \mathrm{MeV}$ [44]. By counting characteristic gamma emission of the activated ${ }^{48} \mathrm{~V}$ a total number of protons with energies $>5 \mathrm{MeV}$ can be determined. Using a multi-layered stack of titanium as the detector for the laser-accelerated ion beam, a rough energy spectrum can be inferred by calcu- 
lating the range-energy response of each successive layer. This technique also allows us to cross calibrate our calculation of the RCF response discussed above.

Using a detector stack of alternating layers ${ }^{48} \mathrm{Ti}$ (75- $\mu \mathrm{m}$ thick) and RCF $(240-\mu \mathrm{m}$ thick$)$, a total number and temperature for the proton distribution can be determined independently by two distinct methods. Such an experiment was performed at the JanUSP laser with 9.2 J of laser energy incident on a 3- $\mu \mathrm{m}$ thick Al foil [45]. Fig. 4.6 shows (a) the integrated energy in the proton beam deposited in each layer of RCF and (b) the measured yield of ${ }^{48} \mathrm{~V}$ atoms in each Ti foil. The measured isotope count has been convolved with the detector efficiency, foil geometry, and half-life to obtain the number of ${ }^{48} \mathrm{Ti}$ activations induced in the foil at the initial time of the laser shot. The data points represent layers of either RCF or Ti, and the horizontal axes has been converted to the minimum proton energy required to register a signal in each specific layer.

The solid lines in Fig. 4.6 are the calculated best fits to the data. In the case of the RCF, the best fit is obtained with $N_{o}=2.3 \times 10^{11}$ and $k T=2.15 \mathrm{MeV}$. For the activated Ti, we find $N_{o}=2.4 \times 10^{11}$ and $k T=2.3 \mathrm{MeV}$. In both cases, an excellent fit over the entire spectrum is obtained. The two independent fits agree well with each other to better than $7 \%$ in both the total proton number and the temperature. This allows us to conclude that our calibration for the response function of RCF is accurate and that the use of a Maxwellian for the proton energy distribution is acceptable at least in the region $>5 \mathrm{MeV}$. It also verifies that the deposited dose in the RCF is 
predominantly from protons, and not due to $\mathrm{x}$ rays or electrons.

Using the best fit values of the proton energy distribution, we can calculate the total kinetic energy of the protons,

$$
\text { Total Proton Energy }=\int_{5}^{E_{\max }} E N(E) d E,
$$

where the limits of integration are between $5 \mathrm{MeV}$ and $E_{\max }$, the maximum proton energy observed on the RCF. The lower limit must be set to $5 \mathrm{MeV}$ for two reasons: (1) The Al blast shield in front of the detector stack plus the first layer of polyester in MD-55 will prevent any protons with energy less than $\sim 5 \mathrm{MeV}$ from reaching the $\mathrm{RCF}$, and (2) the cross section for titanium activation is $\sim 5 \mathrm{MeV}$, which prevents any nuclear activation at lower energies. Substituting the average fit values of $N_{o}=$ $2.35( \pm 0.1) \times 10^{11}$ protons and $k T=2.2( \pm 0.1) \mathrm{MeV}$ into Eq. (4.4) we find the total kinetic energy in the protons to be $63 \mathrm{~mJ}$. This represents a conversion efficiency of $0.66 \%$ of the incident laser energy into protons with energy $>5 \mathrm{MeV}$.

\subsubsection{Neutron Detection}

The art and technique of neutron detection is typically divided into two categories: low-energy neutrons (from thermal energy up to hundreds of keV) and high-energy neutrons (energies of $\mathrm{MeV}$ and greater). Although low-energy neutrons were produced in the experiments presented in the following chapters, our principle interest was

the production and characterization of high-energy neutrons. Specifically, neutrons 


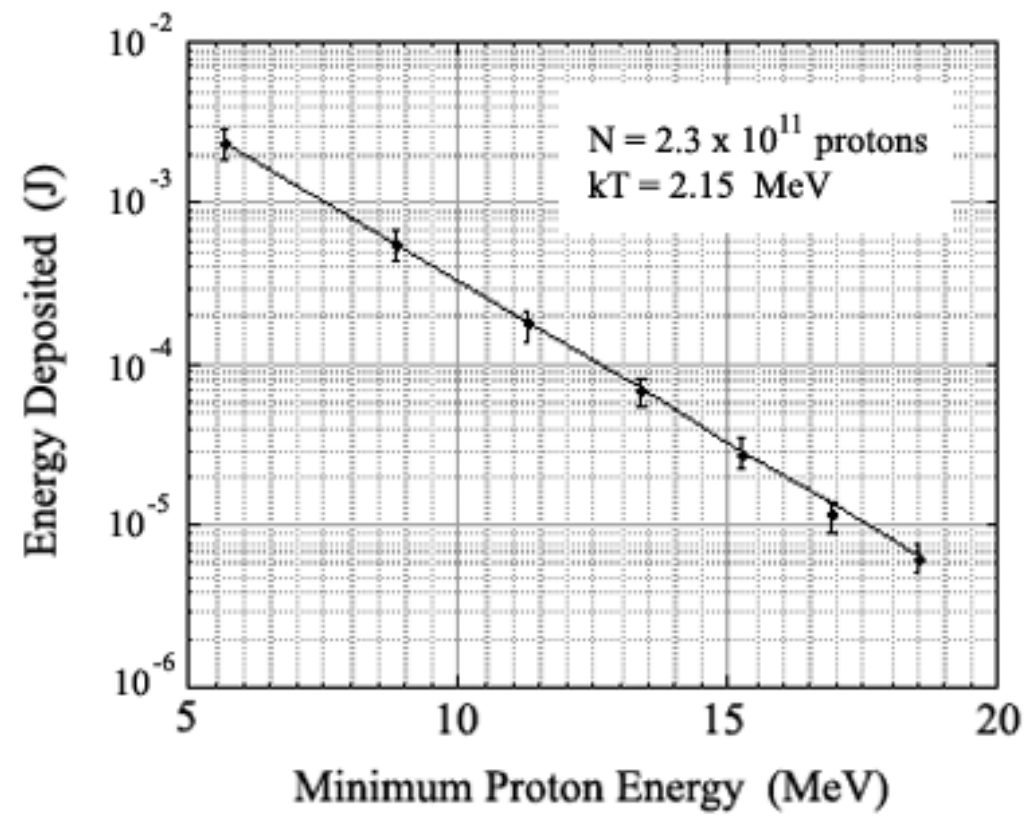

(a)

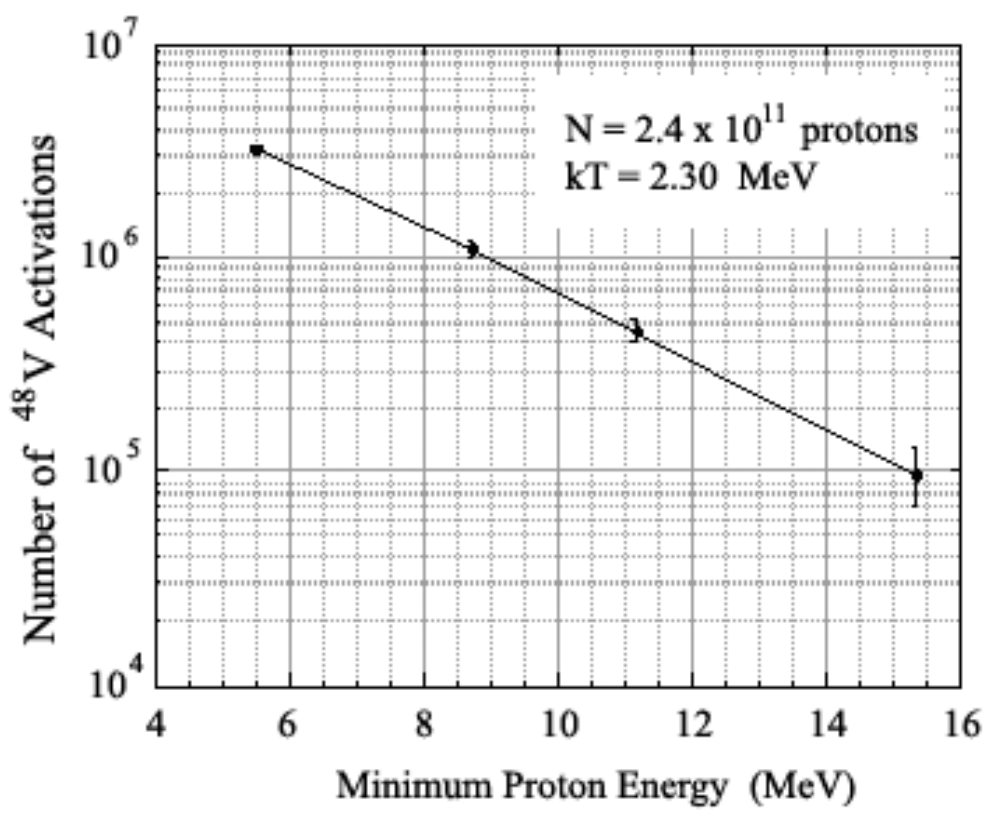

(b)

Figure 4.6: Calculated best fits to the data of (a) the integrated energy in the proton beam deposited in each layer of RCF and (b) the measured yield of ${ }^{48} \mathrm{~V}$ atoms in each Ti foil. Shot parameters: $9.2 \mathrm{~J}$ of laser energy incident on a $3-\mu \mathrm{m}$ thick $\mathrm{Al}$ foil. 


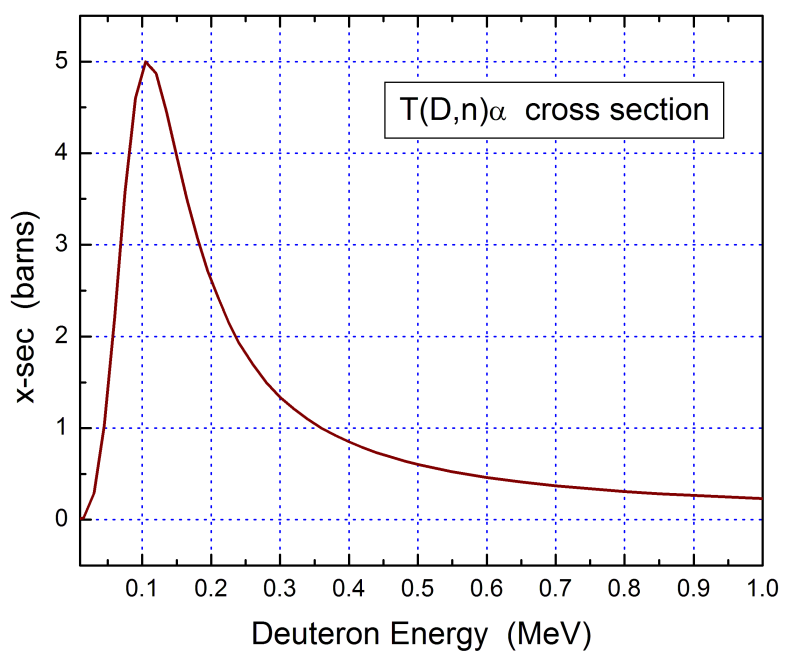

Figure 4.7: The DT-fusion reaction has a peak cross section of $\approx 5$ barns at an energy of $110 \mathrm{keV}$.

produced from the fusion of deuterium and tritium (DT reaction) described by,

$$
\mathrm{D}+\mathrm{T} \longrightarrow \alpha+n \quad Q=+17.6 \mathrm{MeV}
$$

The cross section for the DT reaction is shown in Figure 4.7.

This reaction has one of the highest fusion cross sections of $\approx 5$ barns at an energy of $110 \mathrm{keV}$ [46], which has kept it at the center of most fusion research programs for the past eighty years. The liberated neutron carries away approximately $70 \%$ of the total energy, $14.1 \mathrm{MeV}$. The heavier alpha particle carries the remaining $30 \%$ of the energy, 3.5 MeV. To demonstrate deuteron acceleration and the feasibility of a short-pulse ( $<1 \mathrm{~ns}$ ), 14-MeV neutron source, laser accelerated deuterons were used to irradiate a tritiated-titanium target. An in-depth description of the DT neutron 
experiment is given in Chapter 5. Here we restrict discussion to the method of nuclear activation used to detect high-energy neutrons.

Naturally occurring gold is found entirely (abundance $=100 \%$ ) in the form of the stable isotope ${ }^{197} \mathrm{Au}$. The nuclear reaction ${ }^{197} \mathrm{Au}(n, 2 n){ }^{196} \mathrm{Au}$ has a cross section $>2$ barns at neutron energies of $14 \mathrm{MeV}$. The fact that this cross section is essentially zero up until $8 \mathrm{MeV}$ makes it an ideal choice for the detection of DT-fusion neutrons. When using ${ }^{197} \mathrm{Au}$ as a detector, care must be taken to ensure measured counts are from fusion neutrons and not $\gamma$ rays from the competing ${ }^{197} \mathrm{Au}(\gamma, n){ }^{196} \mathrm{Au}$ reaction. A plot of the relevant gold activation cross sections is shown in Fig. 4.8(a). Small amounts of ${ }^{198} \mathrm{Au}$ will be produced as a result of low energy neutrons from various $(i o n, n)$ reactions with material inside the target chamber and the walls of the target chamber itself.

Samples were counted with absolutely calibrated GeLi detectors at the LLNL Nuclear Counting Facility. The measured isotope count is convolved with the detector efficiency, foil geometry, and half-life of the sample [47]. The long half-life of ${ }^{196} \mathrm{Au} \quad\left(\tau_{1 / 2}=6.18\right.$ days $)$ gives more than sufficient time to acquire data using the laser - which has a 30-minute shot cycle - and deliver it to the counting facility. Samples were counted from one to several days in order to ensure good counting statistics. A typical spectrum from a neutron producing shot is shown in Fig. 4.8(b).

If the contribution from high-energy gamma rays can be properly accounted for and we neglect the possible "down shift" of neutron energies, the number of DT- 


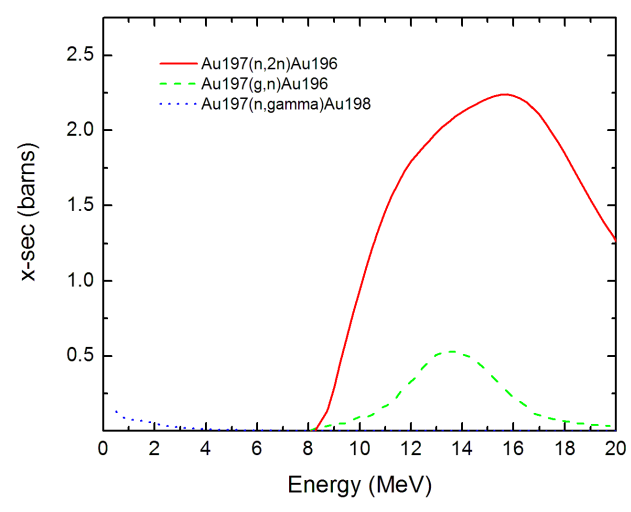

(a)

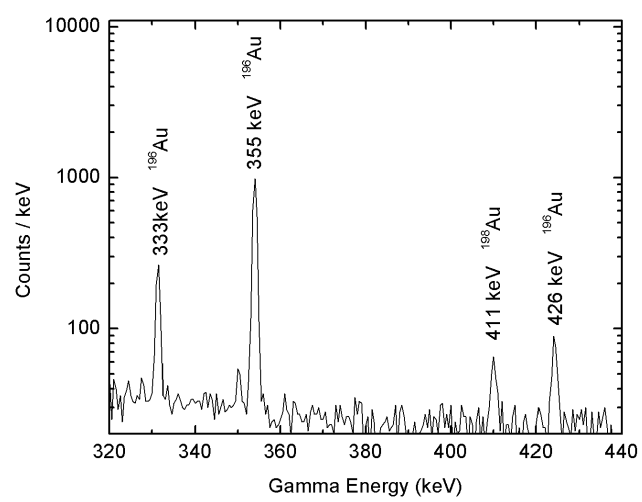

(b)

Figure 4.8: (a) Gold nuclear activation cross sections. Neutrons and gamma rays above $8 \mathrm{MeV}$ will produce ${ }^{196} \mathrm{Au}$. Low energy neutrons will produce ${ }^{198} \mathrm{Au}$. (b) Gamma spectrum from activated gold sample. Three lines are visible from the production of ${ }^{196} \mathrm{Au}\left(\tau_{1 / 2}=\right.$ 6.18 days $)$ and one from the production of ${ }^{198} \mathrm{Au}\left(\tau_{1 / 2}=2.69\right.$ days $)$.

fusion neutrons produced can be estimated by assuming only neutrons of $14.1 \mathrm{MeV}$ contribute to the production of ${ }^{196} \mathrm{Au}$. The neutron yield is then just the measured counts of activated gold atoms divided by the solid angle of the gold sample,

$$
\text { Yield }=\frac{\text { counts }}{d \Omega / \Omega}
$$

The number of deuterons produced can then be calculated by dividing the neutron yield by the probability a deuteron will interact with a triton within a distance $d x$ it travels through a tritiated target,

$$
\# \text { of deuterons }=\frac{\text { yield }}{\int N \sigma_{D T} d x}
$$

where $N$ is the atom density of the tritium target and $\sigma_{D T}$ is the DT-fusion cross section. Measured Au-activation spectra and calculated neutron yields are discussed in greater detail in Chapter 5 . 


\section{Chapter 5}

\section{The DT Experiment}

In this chapter, we present evidence that ions heavier than protons can be accelerated if hydrogenous contaminants that cover the laser target can be removed. We show that deuterons can be accelerated from a deuterated-palladium laser target, which has been radiatively heating to remove contaminants. Impinging a deuteron beam onto a tritiated-titanium catcher could lead to the development of a table-top source of short-pulse, 14-MeV fusion neutrons. Particles (protons and neutrons) are diagnosed using the techniques presented in Chapter 4.

\subsection{Motivation}

It stands to reason that if protons can be accelerated from laser interactions with thin foils, so can heavier ions. To accomplish this, however, protons that are preferentially accelerated due to their high charge-to-mass ratio must be removed. Hegelich et 


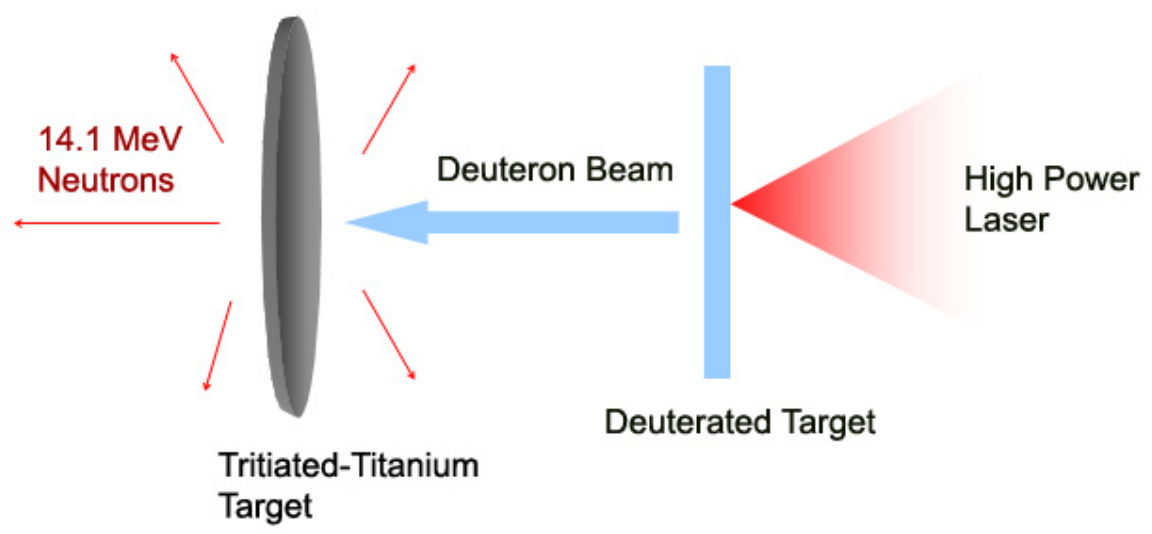

Figure 5.1: Schematic of DT experiment.

al. showed that resistively heating laser targets sufficiently reduced the concentration of hydrogenous contaminants on the target surface. Less hydrogenous contaminants reduced the amount of energy consumed by accelerated protons and allowed for the acceleration of heavier ions from the target bulk material. They reported the acceleration of $\mathrm{F}^{7+}$ ions up to energies of $100 \mathrm{MeV}, \sim 5 \mathrm{MeV} /$ nucleon [48].

Based on previous observations that heavier ions can be accelerated, we attempted to develop a short-pulse source of $14 \mathrm{MeV}$ neutrons by accelerating deuterons into a tritiated-titanium target. The successful production of a short-pulse neutron source could have applications such as neutron radiography and as useful tool in the development of fast neutron diagnostics. A schematic of the experiment is shown in Fig. 5.1. The experiment consists of three main components, each of which are technically challenging: 
- An existing tritiated-titanium target must be adapted to serve as a deuteron catcher inside the laser-target chamber.

- A laser accelerated deuteron beam must be produced with a flux high enough to produce a measurable neutron signal.

- $14 \mathrm{MeV}$ neutrons must be uniquely identified.

Each of these components are discussed in detail below along with results that may indicate successful neutron production are described.

\section{$5.2 \quad$ A Tritiated Titanium Target}

Professor Edward Morse of the Nuclear Engineering Department of UC Berkeley kindly provided a one-inch round, 20-mil thick copper substrate with a coating of $1.25 \mathrm{mg} / \mathrm{cm}^{2}$ of tritiated titanium at a stoichiometry of $\mathrm{TiT}_{1.6}$. The activity of the source in 1983 when it was acquired by Prof. Morse was $4.8 \mathrm{Ci}$. Based on the 12.3 year half-life of tritium, the activity of the source at the time of the experiment was calculated to be $1.6 \mathrm{Ci}$. At this activity, which is not considered small, care must be taken to ensure the tritiated disk can be used without contaminating researchers (i.e. graduate students and laser techs.) and research equipment (i.e. the laser-target chamber and associated vacuum pumps) with radioactivity.

A stainless steel assembly was constructed in which the tritiated-titanium catcher (TT catcher) could be completely enclosed. A simple schematic of the assembly 
Components

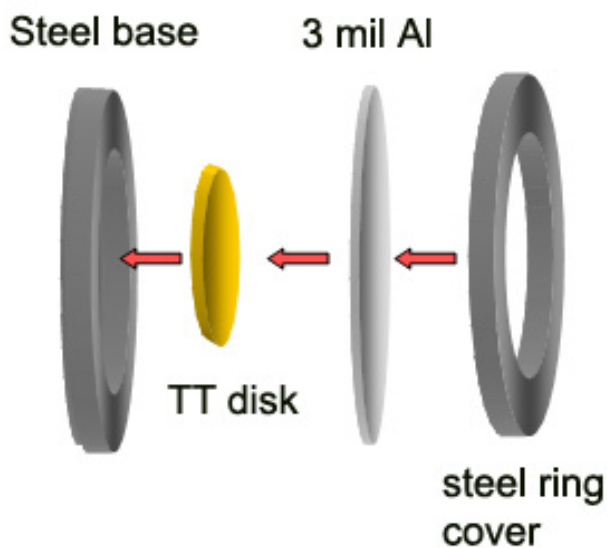

Front View

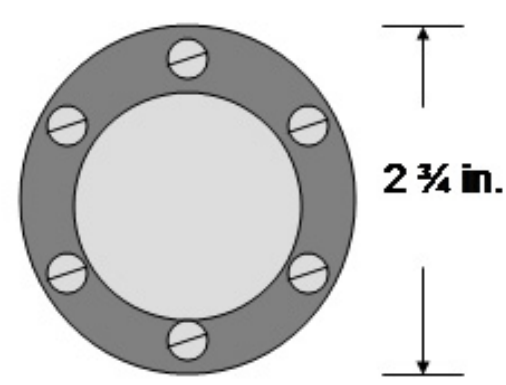

Figure 5.2: Simple diagram of stainless-steel assembly that contains the tritiated-titanium disk used for deuteron catcher in the DT experiment.

is shown in Fig. 5.2. The front face of the assembly must be able to contain the radioactivity but allow the penetration of energetic deuterons. For this reason, a blast shield of 3-mil thick Al was chosen that had sufficient structural integrity to prevent contamination while allowing the penetration of deuterons with energy $>4 \mathrm{MeV}$. Completely assembling the TT catcher at UC Berkeley allowed LLNL to consider the target a sealed source, which eased restrictions on working with radioactivity in the lab. As long as the source is never cracked or damaged it may be used with care by anyone who has had radiological worker training. 


\subsection{Production of a Deuteron Beam}

In order to accelerate deuterons, deuterium must be present in the laser target. Deuterated palladium was considered the best choice for the laser target due to palladium's affinity to the absorption of hydrogenous isotopes and the large difference in the charge-to-mass ratio, $q / m$, between the host and the dopant atom. If, for example, deuterated plastic $\left(\mathrm{CD}_{2}\right)$ had been chosen, the charge-to-mass ratio of various charge states of carbon ions might have competed with the $q / m=\frac{1}{2}$ of the deuteron. A plastic target would also not have been able to withstand radiative heating, a process that was employed to clean the target of contaminants as will be discussed below.

After searching unsuccessfully for commercially available deuterated-palladium foils of thickness $\sim 20 \mu \mathrm{m}$, we decided to fabricate the necessary foils in house. One method of doping, or charging as it is sometimes known, palladium with deuterium is high temperature gaseous diffusion. In this approach, the palladium metal is raised to a high temperature (usually through resistive heating) in a chamber that is then backfilled with deuterium gas. The diffusion constant of palladium is sufficiently high at temperatures $>500{ }^{\circ} \mathrm{C}$ for the gas to diffuse freely through the metal. When the metal cools, the diffusion constant decreases and the gas is trapped as a dopant in the metal lattice $[49,50]$.

The gaseous diffusion method works well and is capable of reaching a maximum dopant stoichiometry of $\mathrm{PdD}_{2}$. Unfortunately, running a current through a chamber filled to 1 ATM with a highly flammable gas is not something for which national 
laboratories like granting graduate students permission, and construction of our own gaseous diffusion chamber was abandoned after several months of bureaucratic effort.

A different approach in which the only safety precautions are latex gloves and protective goggles is electrolytic charging. In this method, metal foils are used as the anode and cathode of a voltage source. When the anode and cathode are submerged in water, a small current passes between them that is sufficiently strong to disassociate the water molecule into positive and negative ions,

$$
\mathrm{H}_{2} \mathrm{O} \longrightarrow \mathrm{H}^{+}+\mathrm{OH}^{-} \text {. }
$$

Once liberated, the $\mathrm{OH}^{-}$ion is attracted to the anode and the $\mathrm{H}^{+}$ion is attracted to the cathode. A schematic of the electrolytic cell with heavy water substituted for normal water is shown in Fig. 5.3. Foils were cleaned before being placed in the electrolytic cell according to the procedure,

1. one minute, $\mathrm{Na}_{2} \mathrm{OH}$

2. rinse with de-ionized water

3. two minutes, $1 \mathcal{M ~ H C l}$

4. rinse with de-ionized water

5. 30 minutes, soak in electrolytic cell

which is similar to the procedures described in Refs. [51, 52, 53]. When titanium foils were charged, bubbles were apparent on both the anode and the cathode. When 


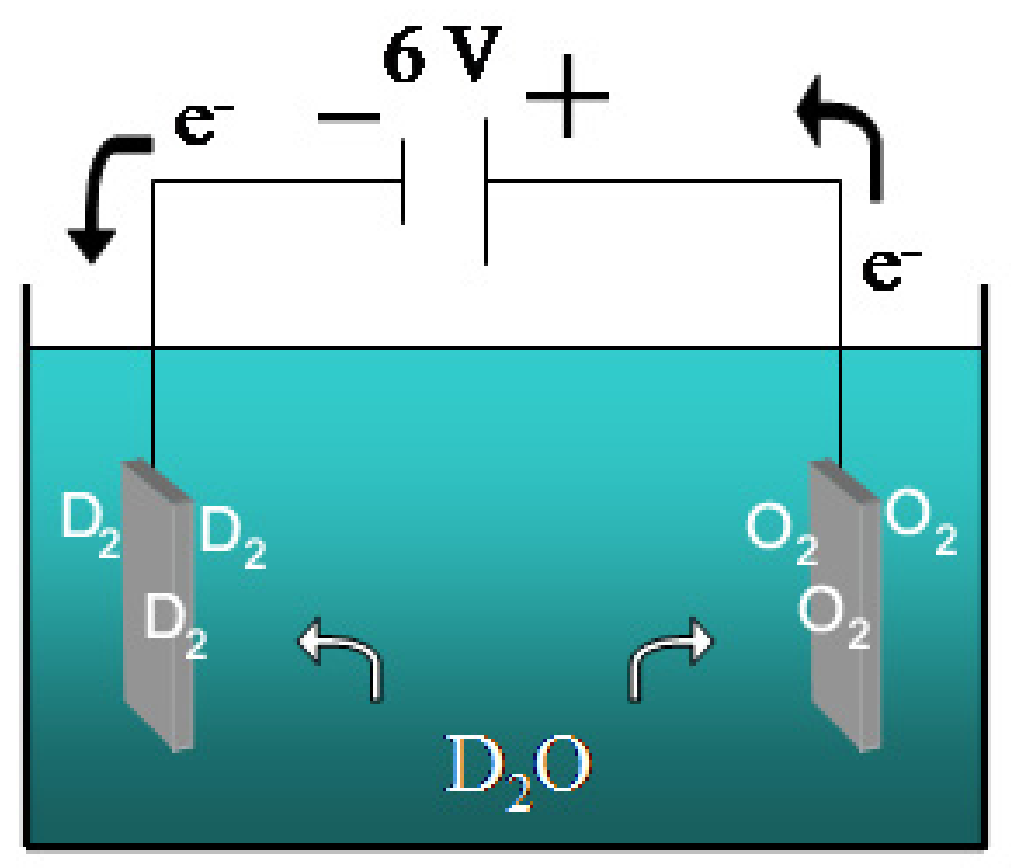

Figure 5.3: Schematic of heavy-water electrolytic cell. Current $(\sim 12 \mathrm{~mA})$ flowing through water is sufficiently strong to disassociate water molecules into separate ions. Negative and positive ions are then attracted to the metal foils acting as the anode and cathode respectively.

palladium foils were charged, bubbles were apparent on the anode, however very few, if any, bubbles could be seen on the cathode. We interpreted this as evidence of deuterium diffusion into the foil attached to the cathode.

To determine the effectiveness of electrolytic charging, a sample from one of the foils was analyzed by Secondary Ion Mass Spectrometry (SIMS). The analysis showed deuterium diffusion into $600 \mathrm{~nm}$ of the material as shown in Fig. 5.4. As shown in the figure, several foils were analyzed. Foils that had no charging and those that were charged with normal water $\left(\mathrm{H}_{2} \mathrm{O}\right)$ showed no deuterium concentration in the material, as expected. 


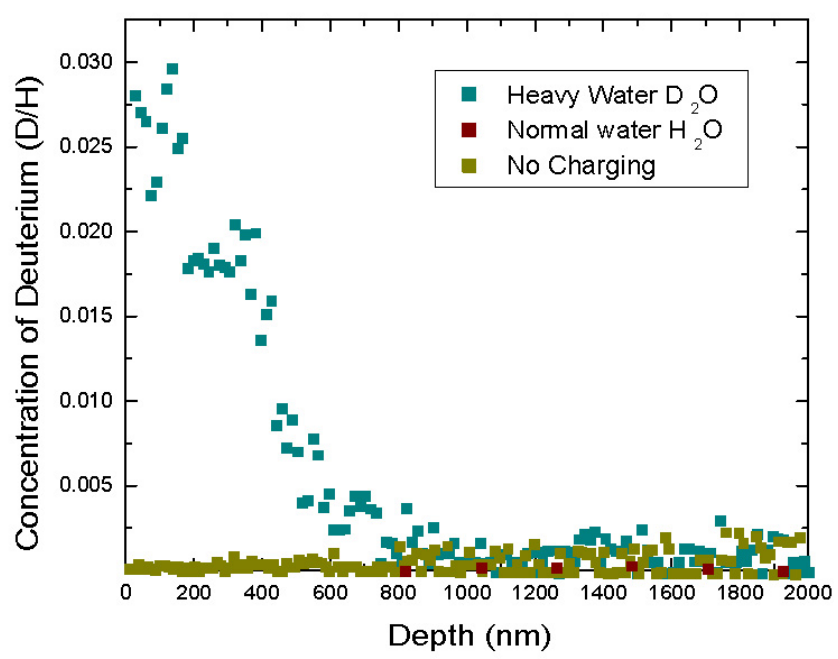

Figure 5.4: SIMS analysis shows deuterium diffused into the palladium foil to a depth of $\sim 600 \mathrm{~nm}$. Charging with normal water and no charging at all showed the expected result of no deuterium concentration in the target material.

Once deuterium is successfully doped into the laser target, we are still left with the problem of removing contaminants from the target surface. Resistive heating, as mentioned at the beginning of this chapter, is not a viable option for removing contaminants in this case. High temperatures associated with resistive heating, $>500^{\circ} \mathrm{C}$, would cause outgasing of the dopant from the palladium foil. To prevent this, we employed a small radiative heater, which we hoped would raise the surface of the target to a temperature sufficient to drive off hydrogenous contaminants without resulting in total loss of the dopant. A schematic of the radiative heater in close proximity to the laser target is shown in Fig. 5.5. A thermocouple on the back surface of the target showed the temperature of the foil was $\sim 250^{\circ} \mathrm{C}$. 


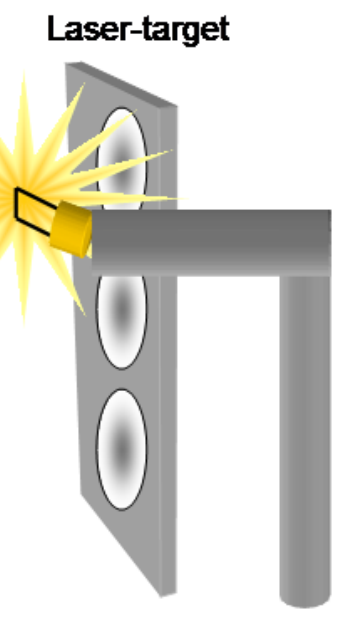

Figure 5.5: $40 \mathrm{~W}$ light bulb filament used as radiative heater, in close proximity $(2-3 \mathrm{~mm})$ to laser target. Thermocouple showed temperature of foil was $\sim 250^{\circ} \mathrm{C}$.

The effectiveness of radiative heating as a tool for removing target contaminants was determined by radiating palladium foils charged with normal water, $\mathrm{H}_{2} \mathrm{O}$. Normal water was used instead of heavy water since RCF was the principle detector and the film's response to protons rather than deuterons was absolutely calibrated. The results from one series of shots is shown in Fig. 5.6. The data shown in part (a) of the figure shows a normal proton beam from a $25-\mu \mathrm{m}$ thick Pd foil. Protons are apparent up to the tenth piece of RCF indicating a maximum proton energy $>20 \mathrm{MeV}$. Part (b) shows the result of radiatively heating a $\mathrm{Pd}$ foil of the same thickness. A blurry radiograph of the filament from $\mathrm{x}$ rays and or electrons is visible on the RCF, and protons are only visible up to the third layer of film or roughly $8 \mathrm{MeV}$. Part (c) of the figure shows the result of radiatively cleaning a foil that has been charged with hydrogen. The radiograph of the filament in this case is much clearer due to the 
(a)

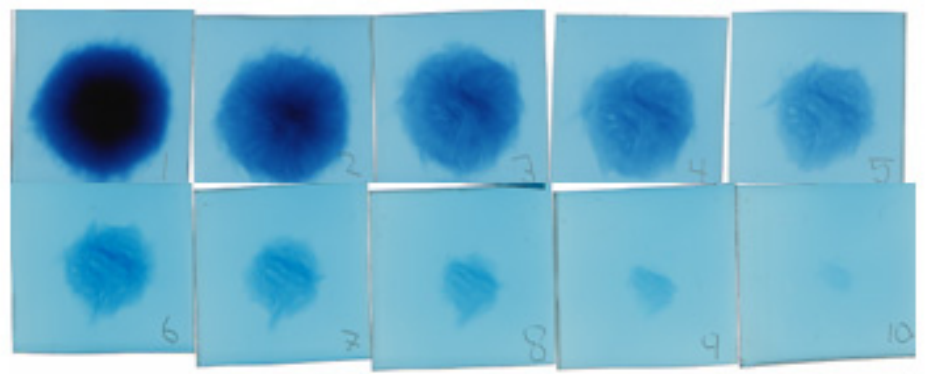

(b)

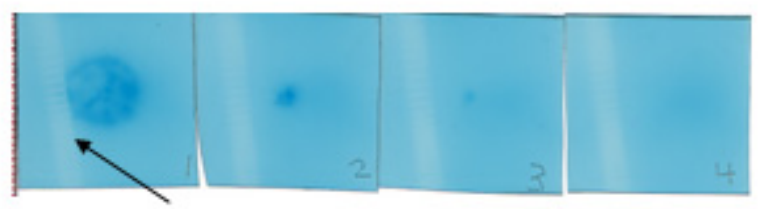

Radiograph of Heating Filament

(c)

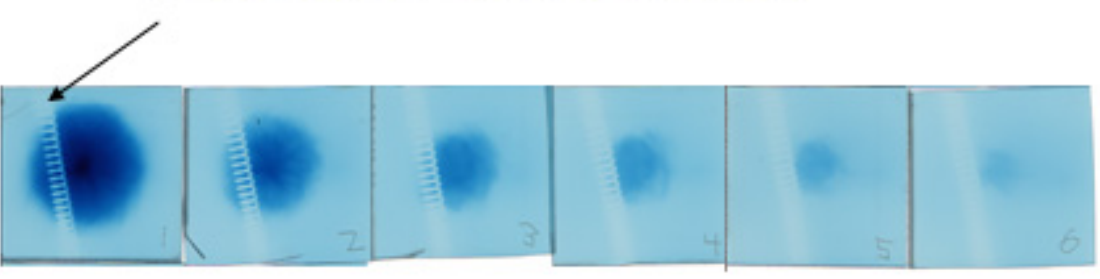

Figure 5.6: Shot series on 25- $\mu \mathrm{m}$ thick Pdfoils, described in detail in the text. RCF on all shots was of type MD-55. (a) Shot\# 10011605, $E_{L}=10.7 \mathrm{~J}$, Maximum ion $>20 \mathrm{MeV}$. (b) Shot\# 10021550, Heated, $E_{L}=10.7 \mathrm{~J}$, Maximum ion $>8 \mathrm{MeV}$. (c) Shot\# 10021630, charged with $\mathrm{H}$, Heated, $E_{L}=9.7 \mathrm{~J}$, Maximum ion $>13 \mathrm{MeV}$.

higher resolution of the proton beam as a radiography tool. Protons are visible up to the sixth layer of RCF indicating a maximum energy $>13 \mathrm{MeV}$. The fact that protons are not visible in a large dose in the data shown in part (b) of the figure and yet returned in the doped target, can be taken as evidence that those shown in Fig. 5.6(c) are from the dopant, not surface contamination.

Quantifying the data using the range-energy-deposition code described in Section 4.1, we can determine the temperature of the proton distribution, $k T$, and the 


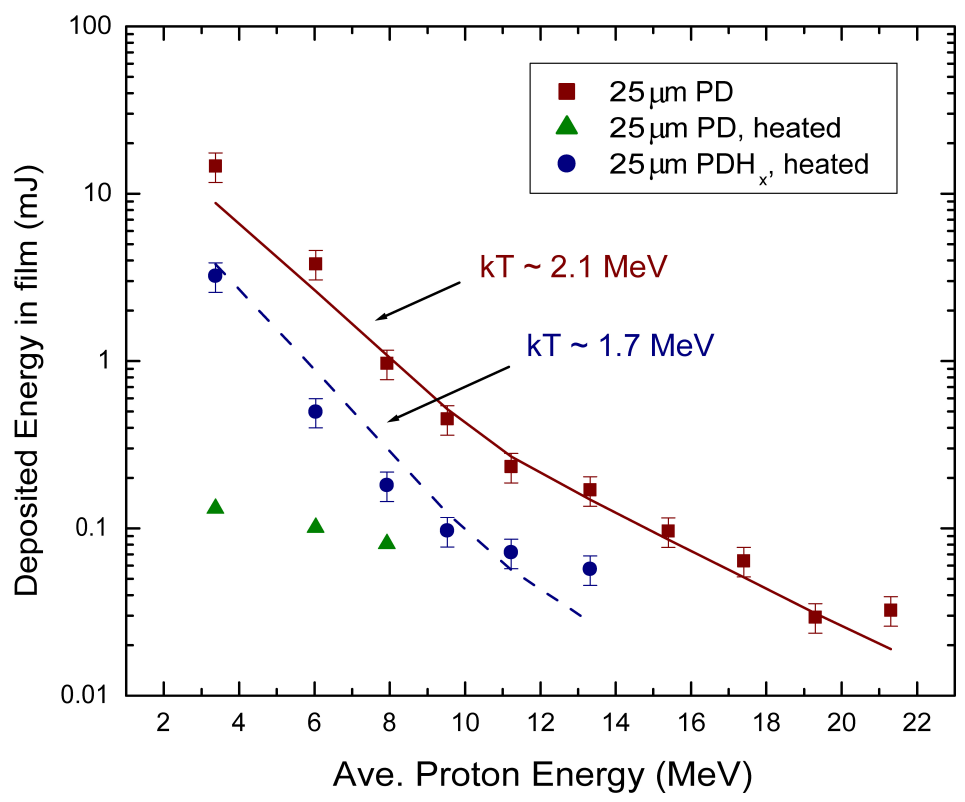

Figure 5.7: Quantitative analysis of proton beam from $\mathrm{Pd}$ targets shows proton yield significantly reduced when un-doped targets are radiatively heated. The reappearance of the proton beam from a heated, doped target $\mathrm{PdH}_{x}$ shows acceleration of doped protons instead of surface contaminants is possible.

total number of protons accelerated. The results of our analysis are shown in Fig. 5.7. For the unheated, uncharged $25-\mu \mathrm{m}$ thick $\mathrm{Pd}$ target, corresponding to the data shown in Fig. 5.6(a), we determined the $k T$ and total proton number to be $2.1 \mathrm{MeV}$ and $3 \times 10^{11}$ respectively. This represents approximately $1.4 \%$ of laser energy converted into kinetic energy of protons. The deposited dose in the film from the heated $\mathrm{Pd}$ target was too low to fit a Maxwellian distribution. However, we can see from the figure, the proton yield is lower by several orders of magnitude. The proton beam from the heated, hydrogen charged target, $\mathrm{PdH}_{x}$, corresponding to the data shown in Fig. 5.6(c), was partially obscured by the heating filament. This slightly decreased 
the measurable yield, but still allowed a sufficient dose to fit to a Maxwellian distribution. For this shot, we determined the proton beam to have a $k T$ and total proton number of $1.7 \mathrm{MeV}$ and $1.5 \times 10^{11}$ respectively and approximately $0.6 \%$ of the laser energy.

At this point, it would appear to be a simple matter of substituting a foil charged with deuterium rather than hydrogen to create an energetic deuteron beam. However, due to several uncertainties, the actual experiment was more challenging than it initially appeared as will be discussed in the next section.

\subsection{Neutron Detection}

The tritiated-titanium catcher was placed $54 \mathrm{~mm}$ behind the Pd foil. The JanUSP laser was incident on the foil at an angle of $22^{\circ}$. Gold disks approximately one-inch round and 0.02-inch thick were placed 4 inches directly behind the catcher. The results of two laser shots, one with a deuterated target and the tritium catcher in place and one without, are shown in Fig. 5.8.

As described in Chapter 4, the only possible mechanism for the production of the $411 \mathrm{keV}$ line of ${ }^{198} \mathrm{Au}$ is the low energy neutron absorption by ${ }^{197} \mathrm{Au}$. Low energy neutrons are produced on every shot from $(i o n, n)$ or $(\gamma, n)$ reactions caused by the accelerated beam or gamma rays interacting with the chamber walls. This peak had the same yield, $\sim 1.4 \times 10^{4}$ counts on both shots as we would expect since its production threshold is unaffected by high energy neutrons. 


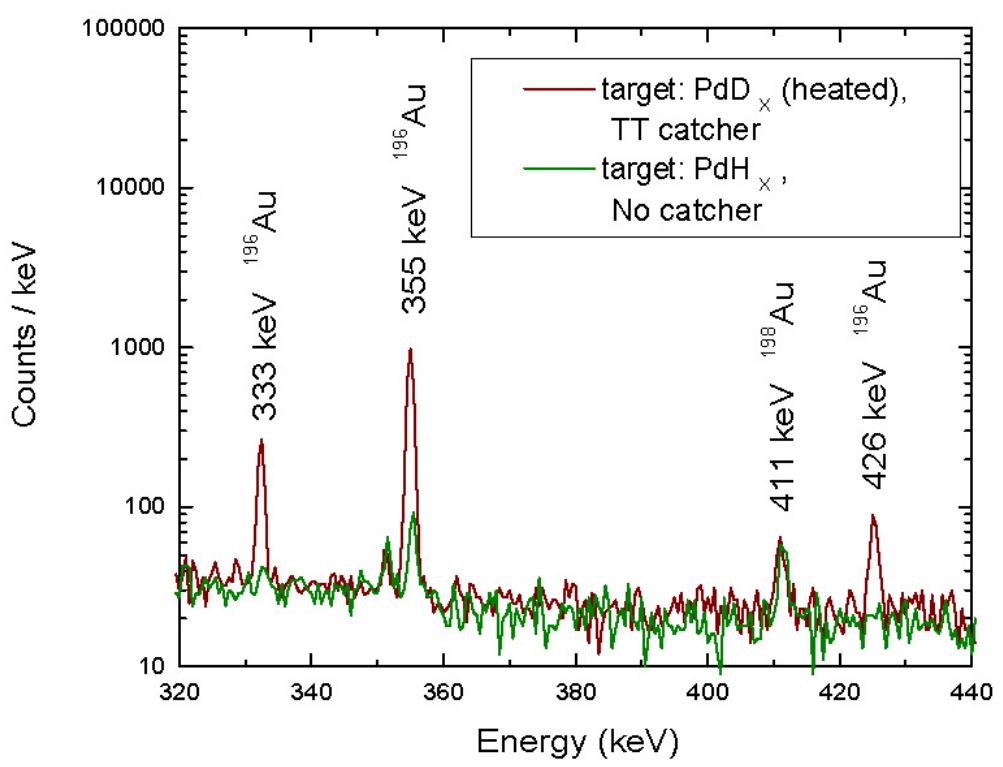

Figure 5.8: Gold activation data. Red trace shows the activation of Shot\# 10311732, radiatively heated deuterated-palladium target with TT catcher in place, laser energy 8.5 J. Green trace shows the activation of Shot\# 11061520, no deuterium or tritium in chamber, laser energy $13.7 \mathrm{~J}$.

There are two possible sources for the production of ${ }^{196} \mathrm{Au}$ : the $(n, 2 n)$ reaction from high energy neutrons and the $(\gamma, n)$ from high energy gamma rays. The yield of ${ }^{196} \mathrm{Au}$ from the DT shot (shown as the red trace in Fig. 5.8), was $\sim 6.02 \times 10^{5}$ counts. This is approximately a factor of 20 higher than the control shot (shown as the green trace), which had a yield of $\sim 3.38 \times 10^{4}$ counts. A factor of 20 is higher than could expected from a shot-to-shot variation in the production of gamma rays from the laser-matter interaction. Especially when we consider the laser energy was higher on the control shot which would suggest a higher gamma flux. We therefore conclude that the increase in ${ }^{196} \mathrm{Au}$ activation on the DT shot is evidence of high energy neutrons.

Furthermore, if we presume the control shot to be a measure of the gamma-ray 
background present on an average shot, we can subtract the yield of the control shot from the yield of the DT shot and calculate how many deuterons would be necessary to produce the measured signal. Using Eq. (4.6) we can calculate the number of neutron yield to be $3.6 \times 10^{7}$. Substituting this number into Eq. (4.7) tells us that $1.1 \times 10^{12}$ deuterons are necessary to produce the measured gold activation counts.

This number of deuterium ions, $1.1 \times 10^{12}$, with energy sufficient to penetrate the $\mathrm{TT}$ catcher blast shield, $>4 \mathrm{MeV}$, would constitute approximately $8.3 \%$ of the laser energy. Although this number is within the realm of possibilities, it is highly unlikely. To date, the highest conversion efficiency of laser energy into energetic ions is $\sim 7 \%$, reported from the Petawatt Laser experiments described in Section 3.3.3. Conversion efficiency can be qualitatively correlated with laser energy. It is unlikely that the JanUSP laser - with an average laser pulse energy of $10 \mathrm{~J}$ - could have a higher conversion efficiency into energetic ions than the $\sim 500 \mathrm{~J}$ pulse energies achievable with the Petawatt Laser.

The experiment had several uncertainties that made it difficult to quantitatively repeat. First, and foremost, radiative heating is simply not a good method for cleaning target surfaces. The temperature laser targets reached during heating was very sensitive to the distance between the target and radiative heater. Even millimeter sized perturbations in the target-to-heater distance resulted in temperature differences in tens of degrees. Therefore, it was never clear that targets could be repeatably raised to the same temperature. 
It was also never clear that targets retained the same amount of deuterium/hydrogen charge after heating. This brings us to a second uncertainty, target fabrication. In laser-plasma experiments, results can only be as good as the target. In this experiment, targets were doped with deuterium/hydrogen by electrolytic charging. This method was selected over the tried and true method of high-temperature gaseous diffusion because of the reduced safety and bureaucratic constraints associated a desk-top, low-temperature, low-voltage facility. In retrospect, electrolytic charging may not be sufficient to achieve the necessary dopant levels. Certainly, a target doped to the stoichiometric limit $\left(\mathrm{PdD}_{2}\right)$ would have more deuterons available to accelerate than a target doped to only a depth of $800 \mathrm{~nm}$.

A third uncertainty of the experiment was repeatability. Only five shots were taken with gold samples as the detection medium. Of those five shots, only two those presented in Fig. 5.8 - had appreciable signals of ${ }^{196} \mathrm{Au}$. Because the TT catcher was in place behind the target instead of RCF, it was difficult to determine if a shot was successful in producing an ion beam or not. ${ }^{196} \mathrm{Au}$ can detect only gamma rays and neutrons and only if those particles have energy $>8 \mathrm{MeV}$. If the target was not sufficiently cleaned, and energy went into accelerating protons, we would not know. If deuterons were accelerated, but at an energy insufficient to penetrate the blast shield of the TT catcher, again, we would not know.

The experiment did show an increase in gold activation that may be attributed to $14-\mathrm{MeV}$ fusion neutrons. This could be taken as evidence that deuterons were 
successfully accelerated from a deuterated-palladium target. Due to the uncertainties listed above, the results were difficult to quantify and achieve repeatably. Were the experiment to be performed again, a different method of removing surface contamination should be used, targets should be charged to the stoichiometric limit, and a method of simultaneously monitoring protons, deuterons and gamma rays should be employed.

For this procedure to be beneficial as a source of $14-\mathrm{MeV}$ neutrons for material studies and/or neutron radiography, the neutron yield and laser repition rate must be several orders of magnitude larger than currently achievable. For comparison, the Rotating Target Neutron Source (RTNS) at University of California, Berkeley ${ }^{1}-$ commonly used to study the effect of fusion neutrons on various materials - produces $1.0 \times 10^{12}-6 \times 10^{12} \mathrm{n} / \mathrm{s}$. Increasing neutron yield from the DT experiment could easily be done by employing a petawatt laser to irradiate deuterated targets. As described in Chapter 3, petawatt lasers can accelerate $>10^{13}$ protons from typical metallic targets. If the same lasers were used to irradiate highly deuterated palladium targets, it is presumable $>10^{12}$ deuterons could be accelerated. At this level of deuteron yield it would much easier to discern the difference in gamma-activation and that attributed to $14-\mathrm{MeV}$ neutrons in gold. This level of deuteron flux could lead to a comparable amount neutrons per laser-shot. However, to attain a flux similar to the RTNS, a petawatt laser repition rate of $1 \mathrm{~Hz}$ would be required. To date, the

\footnotetext{
${ }^{1}$ For more information on the RTNS please refer the facility website: http://www.nuc.berkeley.edu/fusion/neutron/rtns.html
} 
highest repition rate for a high energy laser is $>30 \mathrm{~min}$. Therefore, even with current petawatt laser technology, a yield of $10^{12}$ neutrons per laser-shot would translate into a flux of $5.5 \times 10^{8} \mathrm{n} / \mathrm{s}$.

An aspect of the DT Experiment that researchers may find attractive is the short time scale of the neutron pulse. Most neutron pulses produced by accelerator based technology - such as RTNS - have pulse lengths on the order of nanoseconds. Only two methods of producing ultra-short neutron pulses $(<1 \mathrm{ps})$ presently exist. The first is know as cluster fusion - pioneered by Todd Ditmire - in which atomic clusters of deuterium gas are irradiated with an ultra-short pulse laser [54, 55]. Cluster fusion experiments have attained DD neutron yields of $10^{5}$ neutrons per Joule of laser energy. The DT experiment described above - if the neutron yeild is to be believed - attained a DT neutron yield of $4.2 \times 10^{6}$ neutrons per Joule of laser energy. Ultra-short neutron pulses could open the door to the regime of time-resolved neutron damage, which is currently studied only through theory and simulation. 


\section{Chapter 6}

\section{Removal of Surface Contaminants}

\section{by Ion Sputtering}

Using an argon-ion sputter gun, contaminants from one side of the laser target can be selectively removed without affecting the other side. Removing contaminants in this way, allows for a direct comparison between the number of protons accelerated from the front surface and those accelerated from the back surface. In this chapter, we present experiments that show how ion sputtering either the front or back surface of a laser target affects the producible proton beam. Both aluminum and gold targets of $15-\mu \mathrm{m}$ thickness were investigated. Based on the experimental results, we conclude that the majority $(>99 \%)$ of high energy protons $(E>5 \mathrm{MeV})$ from the interaction of an ultra-intense laser pulse with a thin foil originate on the back surface of the foil. 


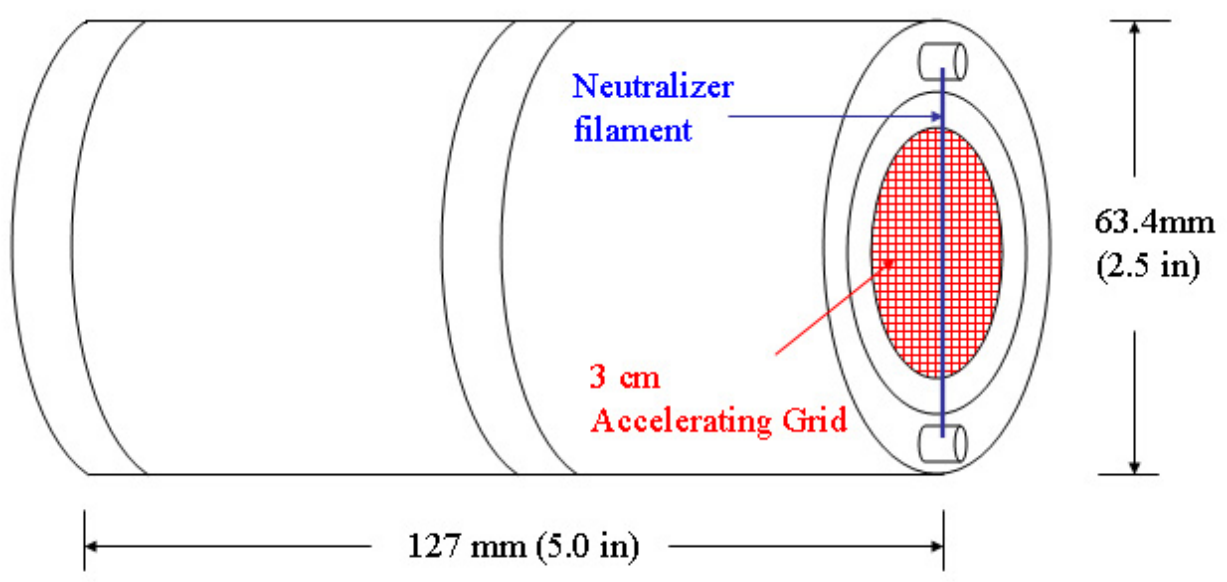

Figure 6.1: Kaufman type, permanent magnet $3 \mathrm{~cm}$ Ion Source

\subsection{The Ion Sputter Gun}

Removing contaminants from laser-target surfaces without raising the temperature of the target material can be a daunting task. Mackinnon et al. used a secondary laser to create a $100-\mu \mathrm{m}$ scale length plasma on the back of the foil, which reduced the maximum proton energy to $<5 \mathrm{MeV}$ and greatly reduced the proton yield [56]. However, using a long-pulse laser to irradiate the back surface is a rather large perturbation of the foil, and it is difficult to isolate the effect of removing the contaminants from the possible laser-plasma interactions caused by the secondary laser pulse. Not to mention, experience has shown that experimental complexity grows in a highly nonlinear manner related to the number of lasers involved. Therefore, the possibility of ion sputtering as a means of reducing surface contamination was embraced.

Laser targets were etched (or "cleaned") with a Kaufman type, permanent magnet 
$3 \mathrm{~cm}$ Ion Source manufactured by Commonwealth Scientific Corporation [57]. A schematic of the ion source is shown in Fig. 6.1. The cleaning of a laser target and the operation of the ion source can be summarized as follows:

1. The target chamber is evacuated to a pressure less than $5 \times 10^{-5}$ torr.

(At this pressure, the monolayer formation rate for $\mathrm{H}_{2} \mathrm{O}$ and $\mathrm{CO}_{2}$ with a near unity sticking coefficient is approximately 15 monolayers/sec.)

2. Argon gas is introduced into the ion source discharge chamber until the pressure in the target chamber measures $\sim 2.4 \times 10^{-4}$ torr.

3. Source cathode emits ion producing electrons.

4. Screen grid forms ions into beamlets.

5. Negative accelerator grid attracts beamlets and forms the ion beam.

6. Neutralizer emits charge balancing electrons.

The neutralizer prevents the target under bombardment from charging up and reflecting ions meant to ballistically collide with contamination molecules.

The ion source was controlled with an IBS 250 Power Supply, a microprocessorcontrolled switching power supply intended to drive gridded ion beam sources [58]. Typical operating parameters for the power supply are shown in Table 6.1. The neutralizer current was kept at the same level as the beam current to ensure charge balance. The target and film-pack holder were both grounded to further prevent 


\begin{tabular}{lc}
\hline \hline & Value \\
& \\
\hline & \\
Carameter & $7.5 \mathrm{~A}$ \\
Discharge Current & $1.5 \mathrm{~A}$ \\
Beam Current & $10.0 \mathrm{~mA}$ \\
Current Density & $1.5 \mathrm{~mA} / \mathrm{cm}^{2}$ \\
Beam Voltage & $500 \mathrm{~V}$ \\
Accelerator Current & $1.0 \mathrm{~A}$ \\
Neutralizer Current & $10.0 \mathrm{~mA}$ \\
& \\
\hline \hline
\end{tabular}

Table 6.1: IBS 250 Power Supply. Typical operating parameters for ion sputter gun charge buildup. An in-situ calibration of the ion-gun etch rate on a thick aluminum foil was measured to be $\sim 170 \AA /$ minute $(\sim 3 \mathrm{AA} / \mathrm{sec})$. Using this value for aluminum, we calculate an approximate etch rate of 1 monolayer/second for the hydrogenous contamination layer. Photographs of the ion gun inside the JanUSP target chamber are shown in Fig. 6.2. 


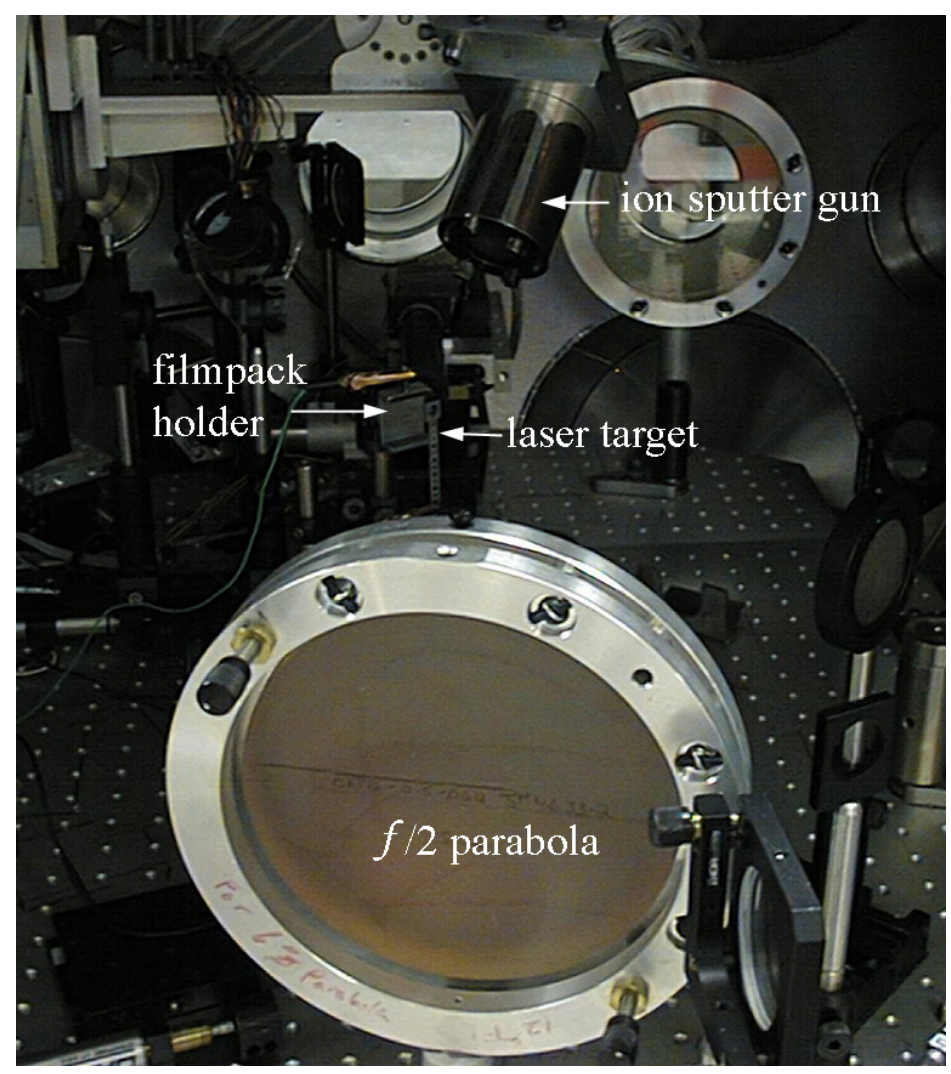

(a)

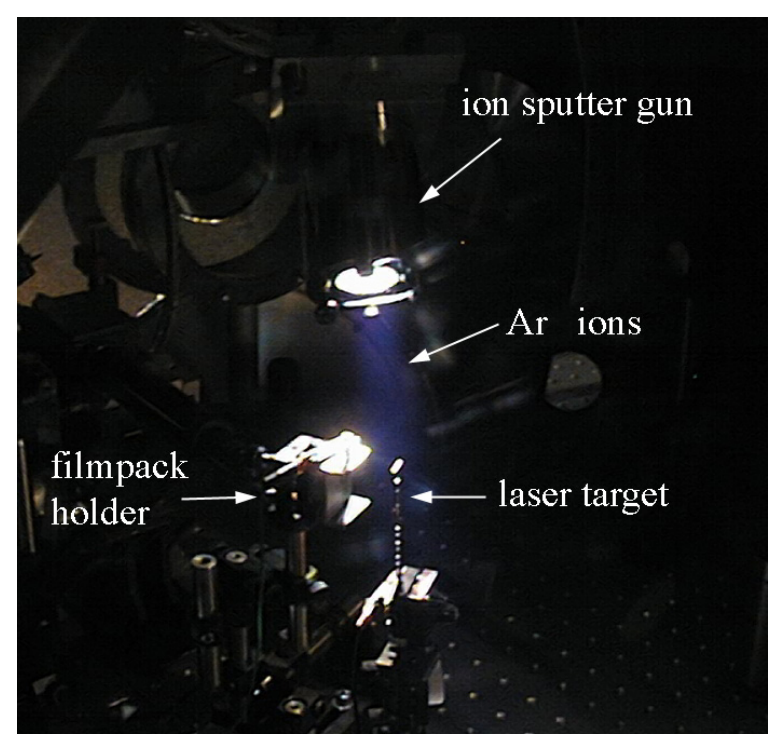

(b)

Figure 6.2: Ion sputter gun shown in the JanUSP target chamber. Source is positioned to etch back surface of the laser target. (a) Ion source is off. (b) Argon ions are visible when source is on. 


\subsection{Surface Cleaning Experiment}

The sputter gun could be positioned to etch either the front surface (laser-interaction side) or the back surface of the laser target with the same gun-to-target distance of $11 \mathrm{~cm}$. The experimental setup with the ion gun positioned to etch the back surface of the target is shown in Fig. 6.3. The vacuum in the experimental chamber was characterized with a residual gas analyzer (RGA) and found to be predominately water vapor. Based on the relative pressure given by the RGA we pressume this water vapor is present at a pressure of $2 \times 10^{-5}$ torr. Hydrocarbon residue (presumably from vacuum pump oil) was present at a lower pressure of $\sim 10^{-7}$ torr. At this pressure, a monolayer of contamination forms very quickly, and continuous cleaning of the target was deemed necessary to ensure the target surface under investigation was free of contaminants. At the time of the experiment, a remote control of the ion gun outside of the laser-interaction room had not been established. Typically shot procedure was

1. Turn on the ion gun.

2. Exit and lock the experiment room, to prevent passers-by from entering and exposing themselves to harmful radiation from the laser-matter interaction at the time of the shot.

3. Take the shot - by firing the laser - while the ion gun is on.

4. Unlock and re-enter the experiment room. 


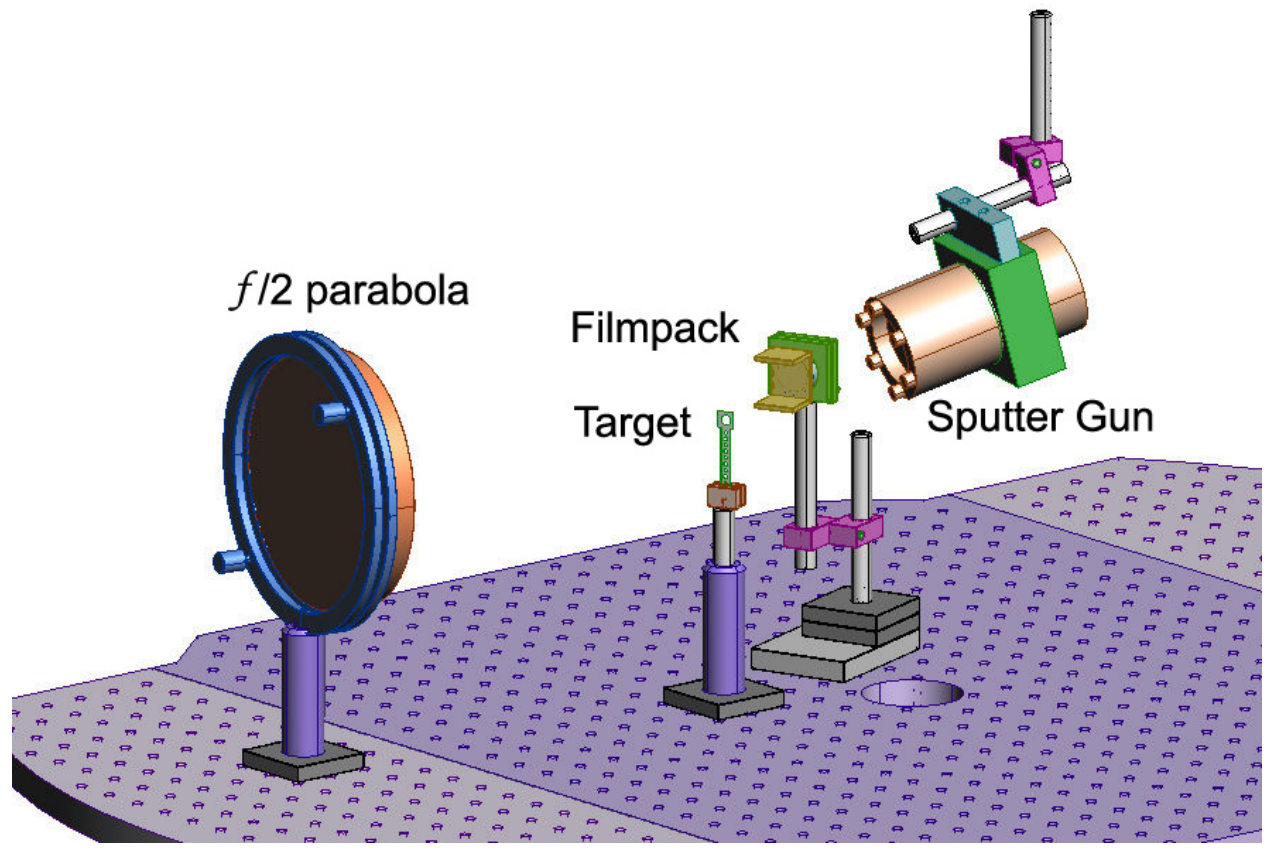

(a)

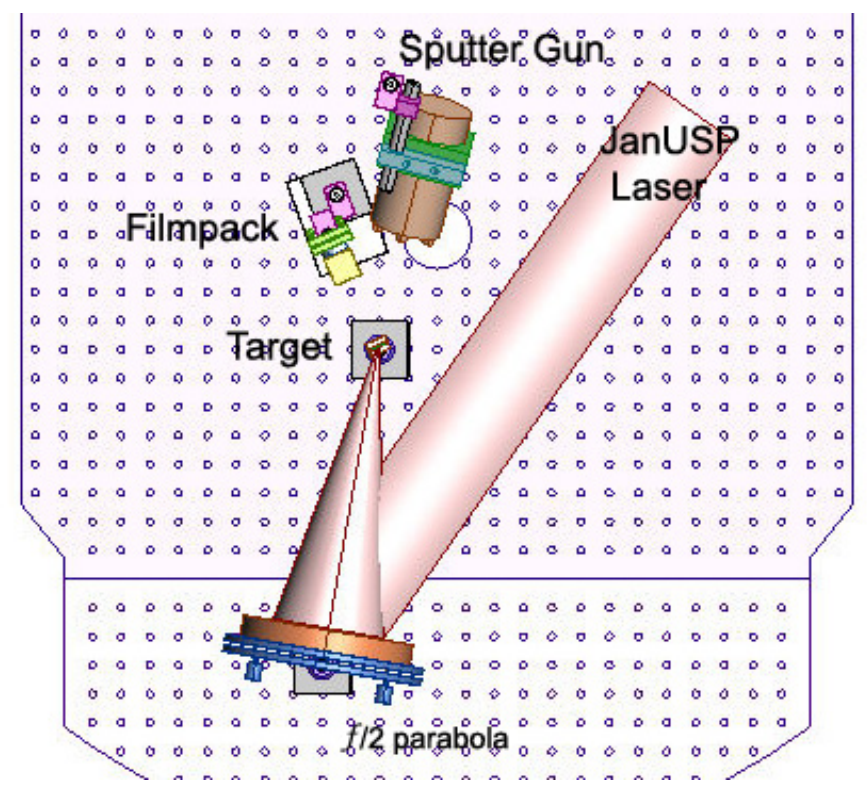

(b)

Figure 6.3: Experimental setup of ion sputter gun cleaning experiment. (a) side view and position of sputter gun when etching back surface of the target. (b) top view with laser beam. 
5. Turn off the ion gun.

6. Vent the target chamber, and retrieve the film.

This procedure limited our minimum etch time to approximately $2 \mathrm{~min}$. The maximum etch time was theoretically unlimited, and on occasion due to technical difficulties with the laser, was longer than desired.

It has been shown that surface modulations on metal foils (present intentionally or otherwise) can imprint modulations onto the accelerated proton beam [59], which appear as lines in the image on the film. These modulations can be used to determine the source size of the proton beam - by counting the number of lines present on the image of the detected beam from a target with a known surface modulation frequency . However, such surface modulations can also serve as points of adsorption for the gas impinging on the target. Rough surface structures on the target can also distort the laminar quality of the accelerated proton beam and complicate interpretation of the film.

To create targets with the smoothest possible surface, Al targets of $15-\mu \mathrm{m}$ thickness were fabricated at LLNL by ion deposition onto glass substrates. Although this process can be expensive - to coat one standard microscope slide cost approximately $\$ 1,000$ - foils can be fabricated with a surface roughness of optical quality, which reduces points of nucleation for contamination and results in a smooth, laminar expansion of the accelerated proton beam. 


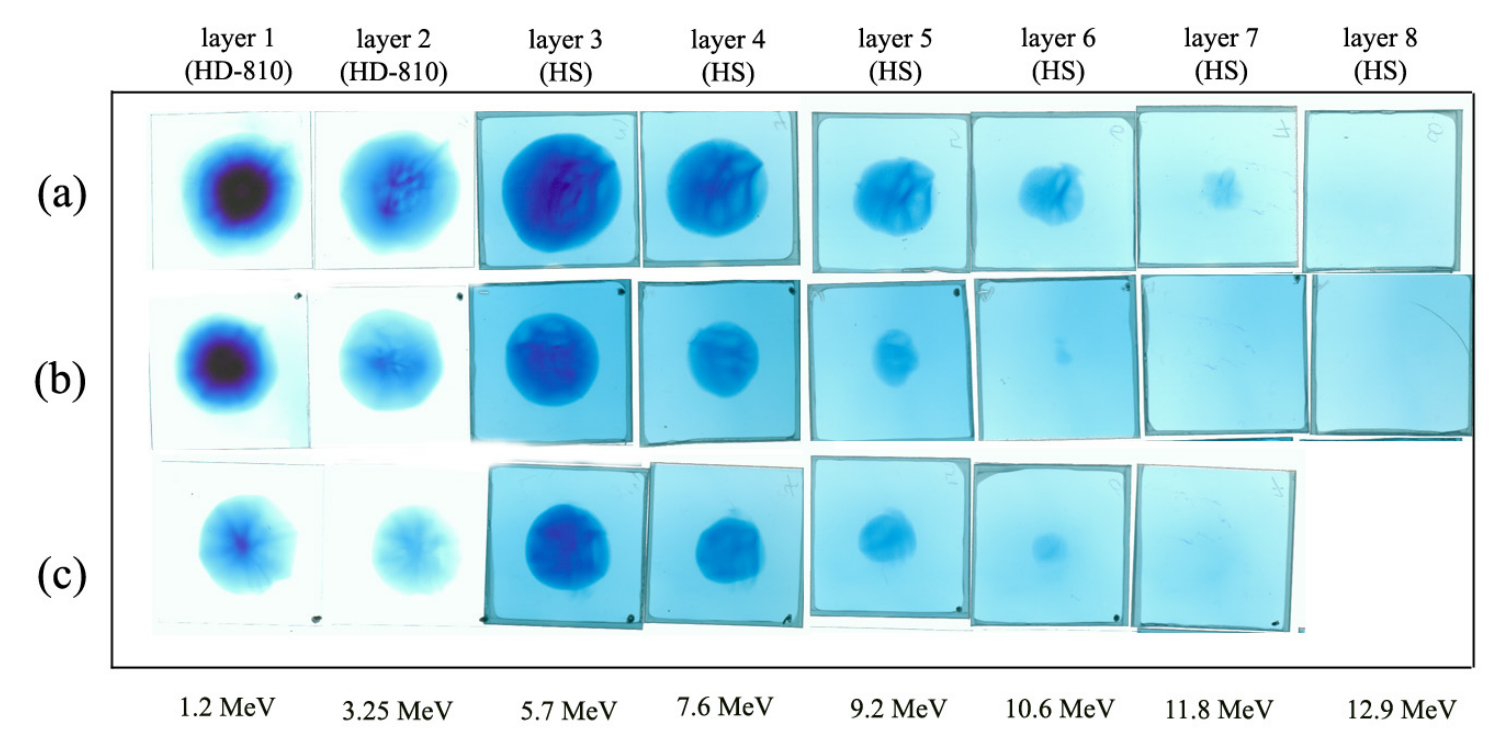

Figure 6.4: Observed proton beam from 15- $\mu \mathrm{m}$ thick $\mathrm{Al}$ targets. Type of film at each layer in the film pack is shown above the data, and average proton energy at that layer is shown below the data. (a) Shot\# 08041850: laser energy of 7.34 J. No ion etching. (b) Shot\# 08042045: laser energy of 7.20 J. Front surface (laser side) of target was etched for $3.2 \mathrm{~min}$. (c) Shot\# 08041810: laser energy of 8.46 J. Back surface of target was etched for 3.3 min.

The results from a typical shot series are shown in Fig. 6.4. All shots shown were taken on the same day at similar laser energies. Fig. 6.4(a) shows a typical proton beam produced by irradiating a $15-\mu \mathrm{m}$ thick Altarget. The effect of etching the target on the front surface or back surface are shown in Figs. 6.4 (b) and (c), respectively. We can see from the data that etching has only a small effect on the proton beam, possibly a small reduction in the total proton yield. This effect does appear to be remarkably different based on which surface - the front or the back - was the etched surface. 

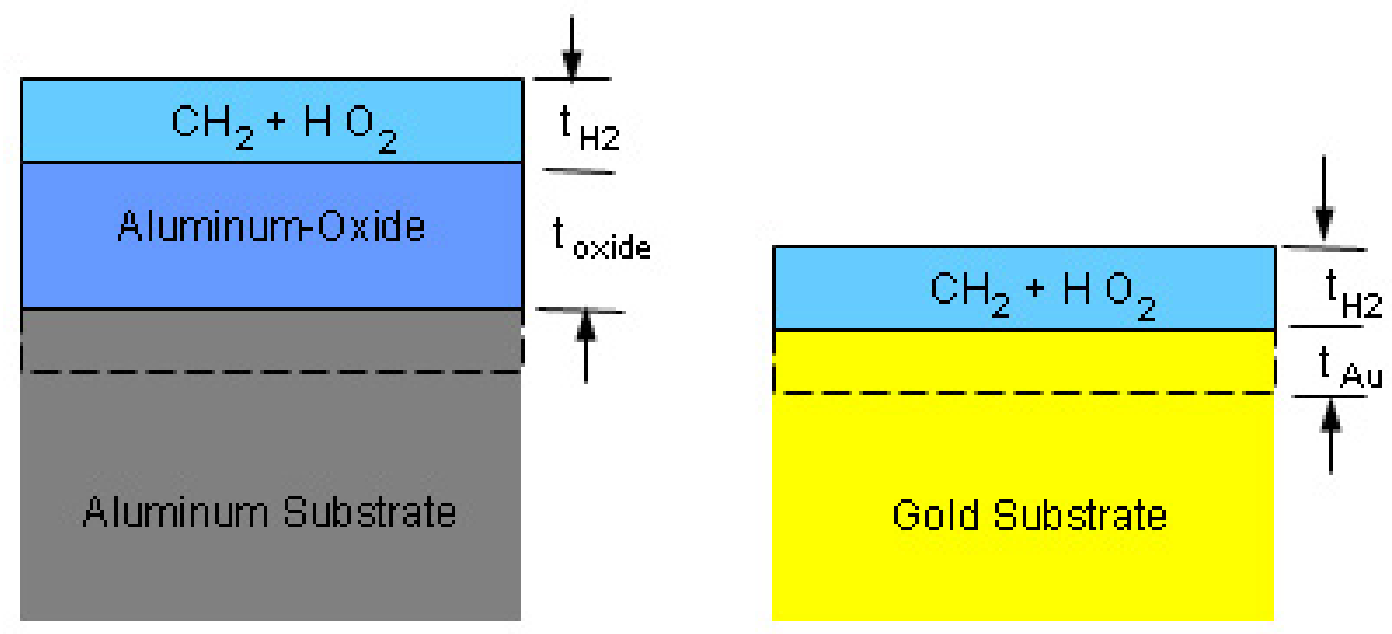

Figure 6.5: X-ray Photoemission Spectroscopy (XPS) showed a 12- $\AA$ thick layer consisting of hydrogenous compounds as well as the metal substrate on both aluminum and gold targets. The aluminum target also possesed a $120-\AA$ thick layer of aluminum-oxide

To understand why ion sputter cleaning has little effect on surface contamination, targets were characterized by X-ray Photoemission Spectroscopy (XPS), which showed a $12-\AA$ thick layer consisting of $23.4 \%$ aluminum, $47.3 \%$ hydrocarbons $\left(\mathrm{CH}_{2}\right)$, and $26 \%$ water vapor $\left(\mathrm{H}_{2} \mathrm{O}\right)$. Below this layer, there also existed a $120-\AA$ thick layer of aluminum-oxide $\left(\mathrm{AlO}_{2}\right)$, as shown in Fig. 6.5. This shows that even after multilayers of hydrocarbons are removed, water vapor in the chamber could react very quickly with the aluminum-oxide layer and trap hydrogen on the surface of the target. It is possible that ion etching does not remove water vapor from the surface faster than it can bond to the oxide layer. A small reduction in the producable proton beam was observed for long etch times, presumably because the oxide layer was completely removed and the adsorption of water vapor onto a pure aluminum surface occurred 
at a slower rate. However, from an aluminum target, the proton beam could never be completely eliminated.

To reduce the effect water vapor had on the laser target, we fabricated targets that were chemically inert to must substances. Using the same ion deposition method, $15-\mu \mathrm{m}$ thick gold foils were produced with optical quality surface roughness. The contamination present on the targets was characterized by XPS, which showed a $12-\AA$ thick layer consisting of $27 \%$ gold, $60.5 \%$ hydrocarbons $\left(\mathrm{CH}_{2}\right)$ and $12.2 \%$ water vapor $\left(\mathrm{H}_{2} \mathrm{O}\right)$, as shown in Fig. 6.5. To ensure the total number of protons observed in experiment is consistent with the number of protons available to the accelerating electric field, we need to calculate the atom density of protons in the contamination layer on the target surface.

We start by finding the thickness of the $\mathrm{H}_{2}$-containing layer. From the XPS analysis, we know the total thickness, $t_{\mathrm{H}_{2}}+t_{\mathrm{Au}}=12 \AA$. The atomic density of gold is $\rho=5.8 \times 10^{22}$ atoms $/ \mathrm{cm}^{3}$. The density of the $\mathrm{H}_{2}$-containing layer is obtained in several steps.

- The $\mathrm{H}_{2}$ density of bulk liquid water is $1 \mathrm{~g} / \mathrm{cm}^{3}$ or $3.2 \times 10^{22}$ molecules $\mathrm{H}_{2} / \mathrm{cm}^{3}$.

- The hydrocarbon has apporximately the density of gasoline, $0.65 \mathrm{~g} / \mathrm{cm}^{3}$.

- The molecular weight of the $\mathrm{H}_{2}$-containing unit $\left(\mathrm{CH}_{4}\right)$ is 14 , so the molecular density of this layer is $0.65 \times 6 \times 10^{23} / 14=2.8 \times 10^{22} \mathrm{moleculesH}_{2} / \mathrm{cm}^{3}$. 
This gives us the average density of the $\mathrm{H}_{2}$-containing layer,

$$
\rho_{\mathrm{H}_{2}}=\frac{0.122 \times 3.2 \times 10^{22}+0.605 \times 2.8 \times 10^{22}}{0.122+0.605}=2.9 \times 10^{22} \text { molecules } \mathrm{H}_{2} / \mathrm{cm}^{3} .
$$

For a unit of area of surface $\left(1 \mathrm{~cm}^{2}\right)$, the volume of gold is $t_{\mathrm{Au}}$; in this volume, there are $t_{\mathrm{Au}} \rho_{\mathrm{Au}}$ atoms of gold. Similarly, the quantity of $\mathrm{H}_{2}$ in the $\mathrm{H}_{2}$-containing layer is $t_{\mathrm{H}_{2}} \rho_{\mathrm{H}_{2}}$. This gives us the ratio,

$$
\frac{t_{\mathrm{H}_{2}} \rho_{\mathrm{H}_{2}}}{t_{\mathrm{Au}} \rho_{\mathrm{Au}}}=\frac{0.73}{0.27},
$$

and using the desities calculated above gives us

$$
t_{\mathrm{H}_{2}}=5.4 t_{\mathrm{Au}} .
$$

Solving Eqs. (6.1) and (6.2) simultaneously we calculate the layer thickness to be $t_{\mathrm{H}_{2}}=10.1 \AA$.

Previous experiments have shown the source size of the proton beam on the back surface of the target to be $\sim 200 \mu \mathrm{m}$ in diameter [59]. The number of $\mathrm{H}_{2}$ units is a source size of $0.02 \mathrm{~cm}^{2}$ is

$$
N_{\mathrm{H}_{2}}=\pi(0.02 / 2)^{2} \times 1.01 \times 10^{-7} \times 2.9 \times 10^{22}=9.2 \times 10^{11} \text { molecules. }
$$

The total number of protons $\left(\mathrm{p}_{\text {total }}^{+}\right)$available to be accelerated by the rear surface electrostatic sheath is simply twice the number of $\mathrm{H}_{2}$ units, $\mathrm{p}_{\text {total }}^{+}=1.8 \times 10^{12}$.

The detected proton beam from irradiating an uncleaned target with an energy of $6.94 \mathrm{~J}$ is shown in Fig. 6.6(a). The data shows a smooth 2-D spatial image of 


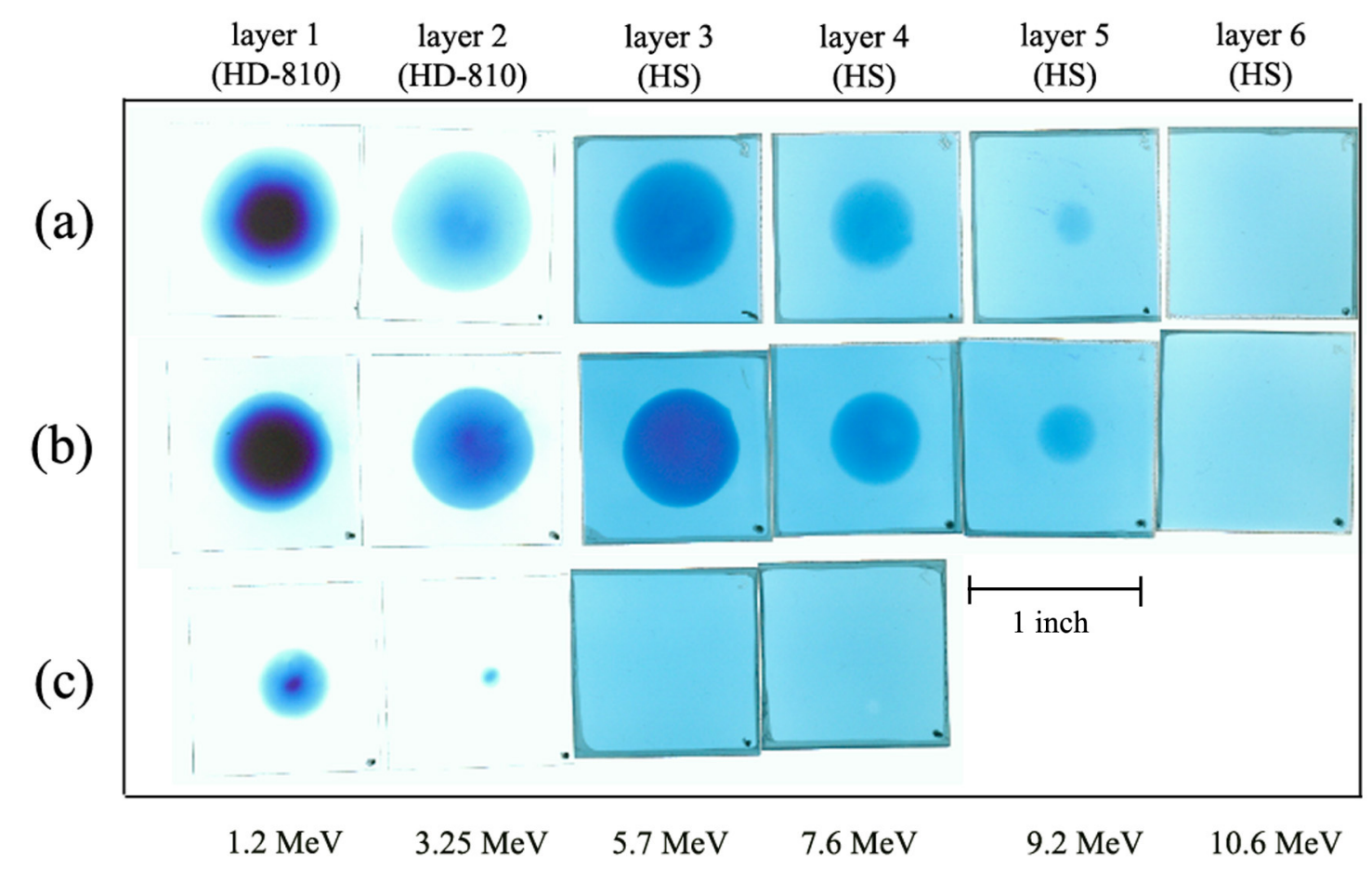

Figure 6.6: Observed proton beam from 15- $\mu \mathrm{m}$ thick $\mathrm{Au}$ targets. Type of film at each layer in the film pack is shown above the data, and average proton energy at that layer is shown below the data. (a) Shot\# 08041535: laser energy of 6.94 J. No ion etching. (b) Shot\# 08042000: laser energy of $8.41 \mathrm{~J}$. Front surface (laser side) of target was etched for 2.6 min. (c) Shot\# 08041630: laser energy of 7.67 J. Back surface of target was etched for 4.0 min.

the proton beam up to a maximum energy of $>9 \mathrm{MeV}$, which was a typical and highly repeatable result for targets of the same thickness at comparable laser energies. Fig. 6.6(b) shows the proton beam from a shot with $8.41 \mathrm{~J}$ on a target in which the front surface was etched for 2.6 minutes. Similar to part (a), the data shows a well collimated proton beam up to a maximum energy of $>9 \mathrm{MeV}$. Etching the back surface of the laser target, however, has a dramatic effect, as shown in Fig. 6.6(c). 


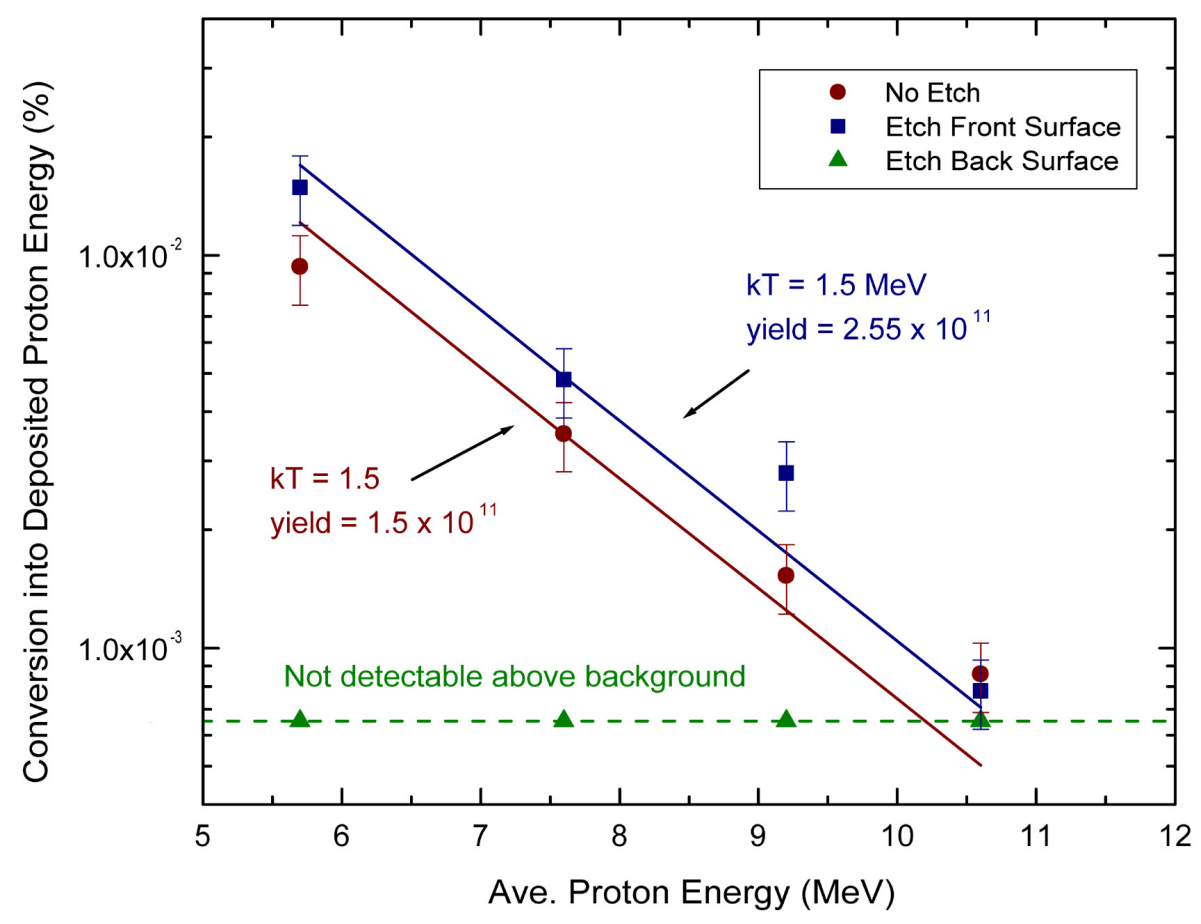

Figure 6.7: Proton spectra from 15- $\mu \mathrm{m}$ thick Au targets fit to a Maxwellian distribution. Shots that were etched only on the front surface or not etched at all show a temperature of $k T=1.5 \mathrm{MeV}$ above proton energies of $5 \mathrm{MeV}$. Shots that were etched on the back surface produced no measurable proton beam above background levels.

This shot had a comparable laser energy of $7.67 \mathrm{~J}$ but was etched on the back surface for 4.0 minutes. Etching the back surface of the target greatly reduced the proton beam number and maximum energy, which made the beam detectable only on the first two layers of RCF. Reduction of the proton beam was highly reproducible on many shots with back-surface etch times of $\sim 2$ min. (corresponding to $>300 \AA$ of material). 
As described in Section 4.1, at energies greater than $5 \mathrm{MeV}$, we fit the proton spectrum to a Maxwellian distribution of the form

$$
N(E)=N_{o} \frac{2}{\sqrt{\pi}(k T)^{3 / 2}} \sqrt{E} e^{-E / k T},
$$

where $N_{o}$ represents the total proton number and $k T$ is the temperature of the distribution in $\mathrm{MeV}$. The energy deposited in each active dye layer of RCF is plotted with the measured dose of the experimental data obtained from the optical density. The proton number $\left(N_{o}\right)$ and temperature $(k T)$ are then adjusted interactively to achieve the best fit with the data. The analysis of the data shown in Fig. 6.6 is presented quantitatively in Fig. 6.7. Above $5 \mathrm{MeV}$, the shot without etching and the shot in which the front surface was etched, both produced a good quality beam with proton yields of $1.5-2.5 \times 10^{11}$ and temperature, $k T=1.5 \mathrm{MeV}$. Etching the back surface of the target produced no measurable proton beam (at $E>5 \mathrm{MeV}$ ) above background levels. This corresponds to a maximum possible yield of $\sim 10^{9}$ for our experimental parameters.

\subsection{Adsorption of Contaminants}

\section{on Target Surfaces}

Why contamination is easy to remove from gold targets, yet difficult to remove from aluminum targets can be qualitatively described with a simple model of physical and chemical adsorption. 


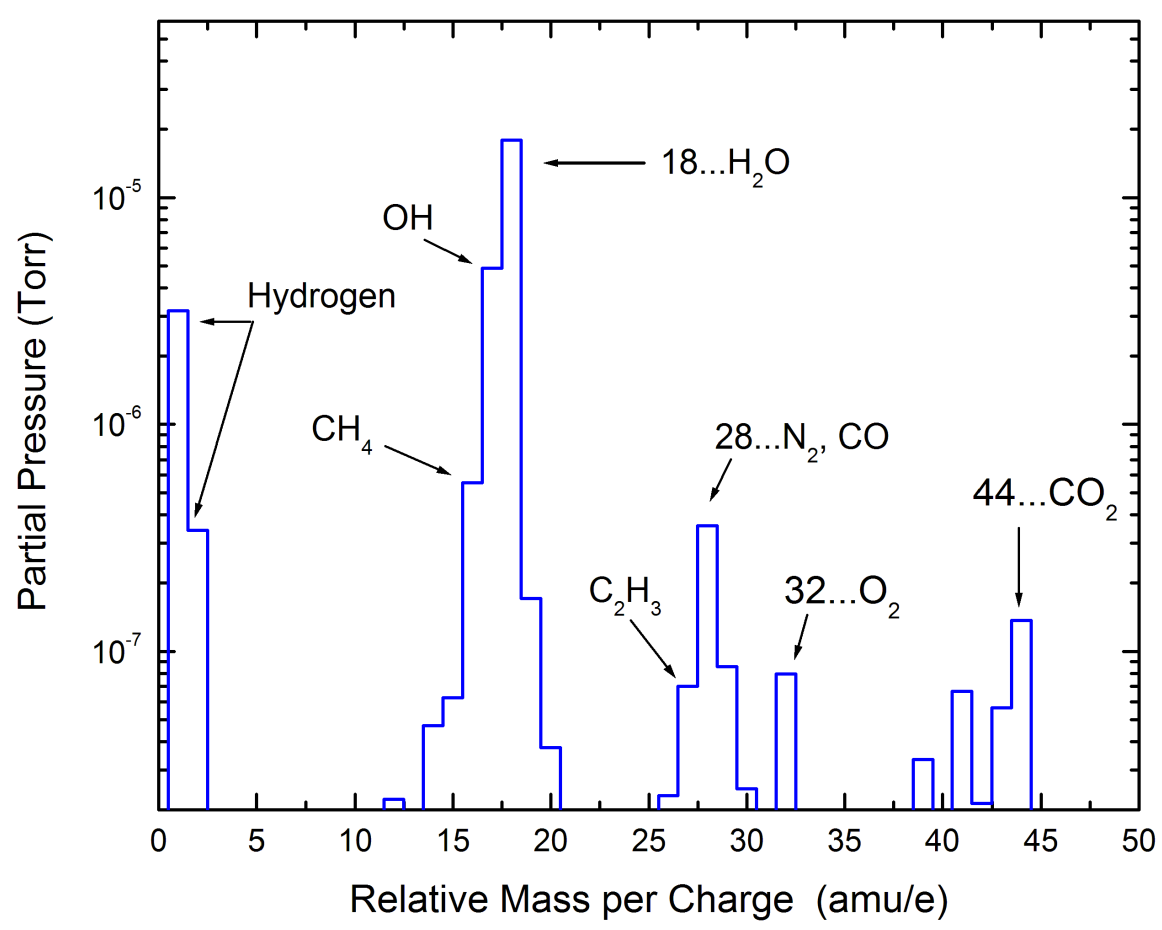

Figure 6.8: Composition and relative pressures of residual gas in JanUSP target chamber.

As described above, the composition and relative pressures of contaminants in the evacuated target chamber were characterized with a Residual Gas Analyzer (RGA), the results of which are shown in Fig. 6.8. We can see from the figure the main source of contamination was predominately water vapor at a pressure of $2 \times 10^{-5}$ torr. Hydrocarbon residue was present at a lower pressure of $\sim 10^{-7}$ torr. This shows that once the ever-present layer of hydrocarbons is removed, only water vapor should contribute to monolayer formation.

Chemical bonding of the water molecule to a solid surface is often thought of in terms of the Lewis dot model, in which the oxygen atom contributes six valence 
Figure 6.9: Lewis dot model of $\mathrm{H}_{2} \mathrm{O}$ molecule. The two lone pairs enable the molecule to interact with other molecules by acting as an electron donor.

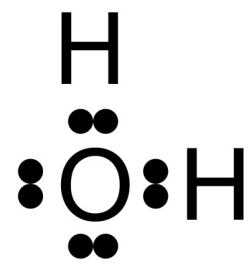

electrons and each hydrogen atom contributes one, as shown in Fig. 6.9. The four valence electrons associated with the oxygen atom represent the two "lone pairs", and the four valence electrons shared between the hydrogen and oxygen atoms represent the intermolecular bonds. The two lone pairs enable the molecule to interact with other molecules by acting as an electron donor. The adsorption of water onto a surface is possible if the lone pair density can interact with either the substrate or neighboring molecules [60, 61].

The breaking of chemical bonds, leading to the dissociation of adsorbed water on some surfaces is possible if the Gibbs free energy of the dissociation products is higher than the that of molecular adsorption. If only one of the internal $\mathrm{O}-\mathrm{H}$ bonds is broken, the products are adsorbed hydroxyl and hydrogen. This type of dissociation into hydroxyl groups is favored in the adsorption of water onto aluminum-oxide surfaces. Water can then form multilayered adsorbed states by hydrogen bonding to the first-layer $\mathrm{H}$ and $\mathrm{OH}$ or $\mathrm{H}_{2} \mathrm{O}$ groups. This high propensity to water vapor adsorption makes removing hydrogenous contaminants from aluminum foils very difficult at a vacuum pressure of $10^{-5}$ torr.

Metallic gold, which is chemically inert, adsorbs water vapor only weakly and only 
at low temperatures. This makes gold targets an ideal choice when investigating the source of accelerated surface contaminants. Once hydrocarbons are removed from a surface by ion sputtering, that surface should remain clean even at room temperature and a vacuum pressure of $10^{-5}$ torr. For a more quantitative description of adsorption with respect to gold and aluminum foils please refer to Appendix A.

\subsection{Simulation of Experimental Results}

To gain insight into the ion acceleration mechanisms present near the target surfaces, we performed 1-D Particle-In-Cell (PIC) computer simulations [62]. This is a good approximation, as the dominant ion acceleration mechanisms are collisionless in nature, due to the fact that the electron sheath for these interactions is typically $>100 \mathrm{keV}$ in temperature. In these simulations, the solid is approximated by a 15- $\mu \mathrm{m}$ slab of plasma with the following characteristics: electron density ramps up from 0 to $60 n_{c r}$ over $1.4 \mu \mathrm{m}$, stays $60 n_{c r}$ for $15 \mu \mathrm{m}$, with an abrupt fall off to $0 n_{c r}$

over $8 \AA$. The increasing density slope roughly models the preformed plasma created by the prepulse and ASE that exist in front of the laser.

To determine the proper charge-to-mass ratio for the heavy, bulk ions we must first determine the electric field present on the back surface of the target. To do this, we must calculate the hot-electron temperature and density. For our laser parameters, the hot electron temperature is given by Eq. (2.48) to be $T_{h o t}=2.9 \mathrm{MeV}$. The hot 
electron density can be approximated by

$$
n_{\text {hot }}=\frac{\varepsilon E_{\text {laser }}}{\text { volume }<E_{\text {electron }}>}
$$

where $\varepsilon$ is the conversion efficiency into hot electrons, $E_{\text {laser }}$ is the laser energy, $<E_{\text {electron }}>=3 T_{\text {hot }}$ is the relativistic average electron energy, and volume denotes the volume of the hot-electron cloud given by the area of the sheath $(\sim 100 \mu \mathrm{m})$ times the target thickness. With a $15-\mu$ m thick target, a conversion efficiency of $20 \%$, and a $T_{\text {hot }}$ of $2.9 \mathrm{MeV}$, Eq. (6.4) predicts an electron density of $n_{\text {hot }}=1.22 \times 10^{25} \mathrm{~m}^{-3}$. Based on this hot-electron density and temperature, we can calculate a hot-electron Debye length of $\lambda_{D}=3.6 \times 10^{-6} \mathrm{~m}$. Substituting these values into Eq. (3.26) we calculate the electric field to be $\vec{E}=8 \times 10^{11} \mathrm{~V} / \mathrm{m}$.

The level of ionization is predicted by the field-ionization-barrier-suppression (FIBS) model [48], which states the $k+$-ionic state will be created when the electric field exceeds the threshold

$$
E_{k}=\frac{U_{k}^{2} \epsilon_{o} \pi}{e Z}
$$

The ionization potentials for the first 15 ionic states of the $\mathrm{Au}$ atom along with the calculated FIBS threshold are given in Table 6.2. Based on the electric field strength calculated above, the bulk ions were taken to have a charge-to-mass ratio of 0.05583 times that of the protons in order to model heavy gold ions at an ionization of $11+$.

There is a thin sheet of protons on the front surface of the target rising to $3 n_{c r}$ over a distance of $0.10 \mu \mathrm{m}$, simulating the preplasma that exists in front of the target due to the laser prepulse. The main laser pulse (peak $I=8 \times$ 


\begin{tabular}{ccc|ccc}
\hline \hline$k$ & $U_{k}(\mathrm{eV})$ & $E_{k}(\mathrm{~V} / \mathrm{m})$ & $k$ & $U_{k}(\mathrm{eV})$ & $E_{k}(\mathrm{~V} / \mathrm{m})$ \\
\hline & & & & \\
1 & 8.3 & $1.2 \times 10^{10}$ & 9 & 131 & $3.3 \times 10^{11}$ \\
2 & 19.6 & $3.3 \times 10^{10}$ & 10 & 149 & $3.9 \times 10^{11}$ \\
3 & 32.3 & $6.0 \times 10^{10}$ & 11 & 169 & $4.5 \times 10^{11}$ \\
4 & 46.2 & $9.3 \times 10^{10}$ & 12 & 248 & $8.9 \times 10^{11}$ \\
5 & 61.1 & $1.3 \times 10^{11}$ & 13 & 270 & $9.8 \times 10^{11}$ \\
6 & 76.8 & $1.7 \times 10^{11}$ & 14 & 293 & $1.1 \times 10^{12}$ \\
7 & 93.3 & $2.2 \times 10^{11}$ & 15 & 316 & $1.4 \times 10^{12}$ \\
8 & 113 & $2.8 \times 10^{11}$ & & \\
\multicolumn{7}{l}{}
\end{tabular}

Table 6.2: Calculated field ionization barrier suppression thresholds for gold. $U_{k}$ is the ionization potential of the $k$ th ionic state [63]. $E_{k}$ is the calculated FIBS threshold field strength.

$10^{19} \mathrm{~W} / \mathrm{cm}^{2}$, pulse length $100 \mathrm{fs}$ FWHM) is incident on the left side of the plasma. An 8-A thick layer of protons on the back surface resembles the hydrogenous contamination layer on the same dimensional scale as existed in the experiment. A schematic of the density profile is shown in Fig. 6.10. The simulation was run for $500 \mathrm{fs}$, at which time no further acceleration of the protons was observed.

We can see from the results of the PIC simulation in Fig. 6.11 that protons from the back surface obtain an energy range 5-14 MeV, which is consistent with the experimental results when the target was not cleaned or cleaned only on the front side as seen in Fig. 6.6(a) and (b). It may be tempting to attribute the well collimated, bright spot in the center of Fig. 6.6(c) to the heavy gold ions from the target bulk material. However, PIC simulations showed the bulk ions reached a maximum energy 


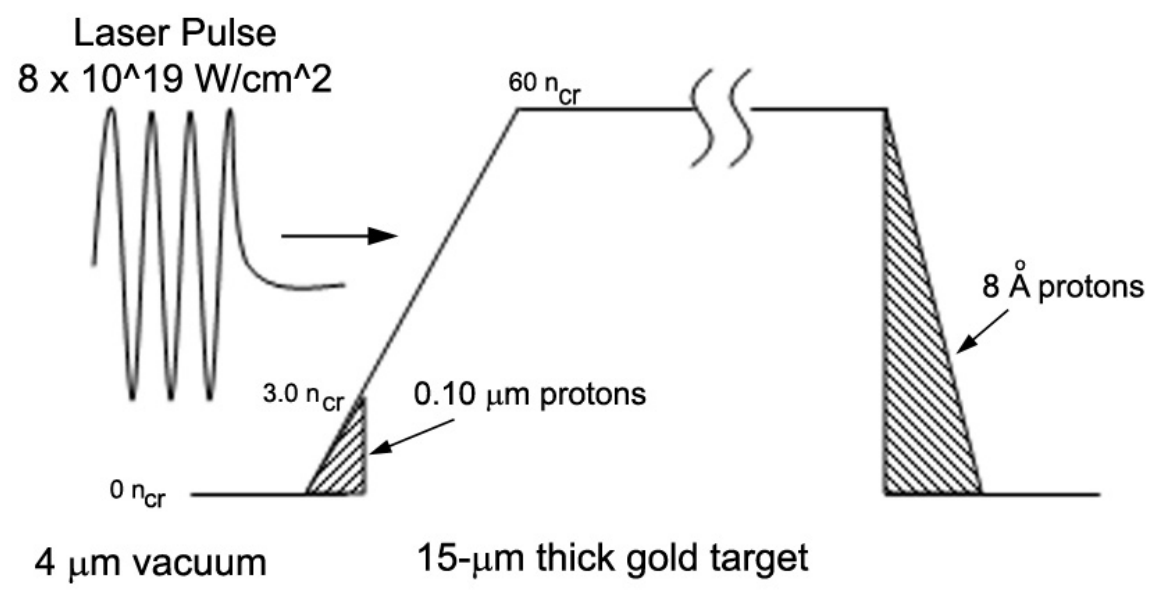

Figure 6.10: Density profiles used in PIC simulation, described in the text.

of $140 \mathrm{MeV}$ even when the back surface protons were removed. SRIM calculations [41] predict that gold ions would need energy $>180 \mathrm{MeV}$ just to penetrate the $18-\mu \mathrm{m}$ thick $\mathrm{Al}$ blast shield in front of the film pack. It is possible, of course, that we were too conservative in predicting the ionization level of gold to only $\mathrm{Au}^{11+}$. A higher level of ionization would increase the final energy given by the PIC model, however, an ionization greater than $11+$ due to field ionization is not expected at the predicted back surface fields of $\vec{E}=8 \times 10^{11} \mathrm{~V} / \mathrm{m}$.

We can also see from Fig. 6.11 that the front surface protons reach a maximum energy of $\sim 4 \mathrm{MeV}$, consistent with the laser ponderomotive potential on the front surface of the target. At this energy, it is conceivable that front surface protons could penetrate the length of the gold target and the aluminum blast shield to deposit their remaining energy $(<3 \mathrm{MeV})$ in the first one or two layers of thin $\mathrm{RCF}$. The fact that 


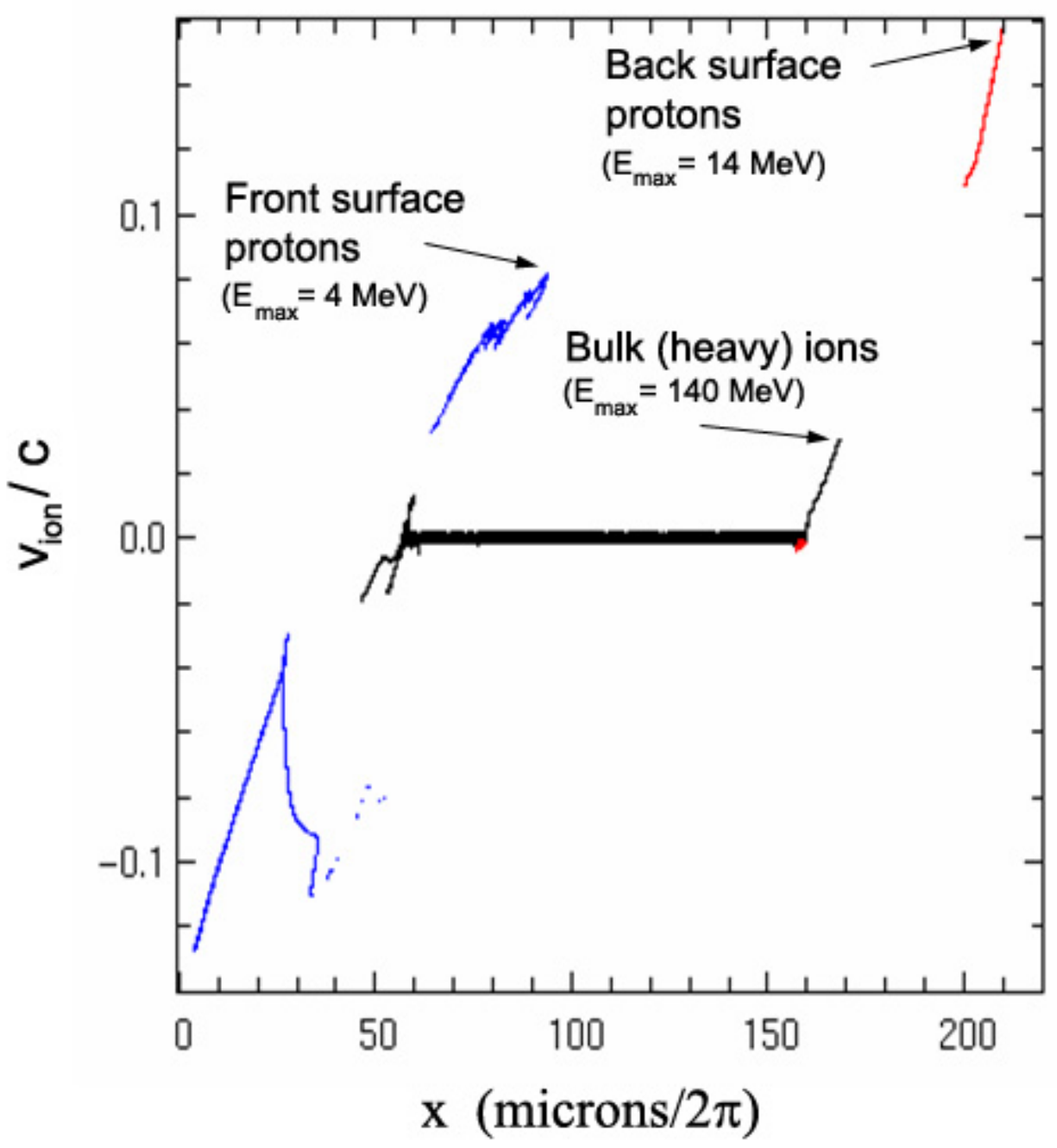

Figure 6.11: Result of 1-D PIC simulation at time, $t=500$ fs. Blue dots show protons from the back surface of the target obtain energies between 5-14 MeV. Red dots show front surface protons reach a maximum energy of $4 \mathrm{MeV}$. Heavy (gold) ions, shown by the black dots, reach a maximum energy of $140 \mathrm{MeV}$. 
the bright center spot of Fig. 6.6(c) is so well collimated, tends to argue against the case for front surface protons which have a broad spatial distribution [37]. Given that the image on the first two pieces of film in Fig. 6.6(c) is in the same position as the images of Figs. 6.6(a) and (b), we attribute the signal to protons on the back surface of the target that were not removed by ion-sputtering. The generated proton beam was decreased in yield to such an extent that no signal was detectable at energies $E>5 \mathrm{MeV}$.

In conclusion, we have shown that irradiating a thin aluminum and gold foils with an ultra-intense laser pulse produces a proton beam with a yield of $>10^{11}$ and temperature, $k T=1.5 \mathrm{MeV}$ with a maximum proton energy $>9 \mathrm{MeV}$. In the case of aluminum foils, removing contaminants from the target surface was difficult due to the adsorption rate of water vapor onto the present aluminum-oxide layer. Aluminum targets had to be etched extensively before we could measure a reduction in the proton yield.

Gold targets, which are chemically inert to water vapor, were more susceptible to ion sputtering and allowed for a direct comparison between the relative contribution to the proton beam from front surface and back surface contaminants. Removing contaminants from the front surface of the laser target, had no observable effect on the proton beam. However, removing contaminants from the back surface of the laser target reduced the proton beam by two orders of magnitude to, at most, a yield of $\sim 10^{9}$ and a maximum proton energy $<4 \mathrm{MeV}$. Based on these observations, we 
conclude that the majority ( $>99 \%)$ of high energy protons $(E>5 \mathrm{MeV})$ from the interaction of an ultra-intense laser pulse with a thin foil originate on the back surface of the foil. Our experimental results are in agreement with PIC simulations showing back surface protons reach energies up to $14 \mathrm{MeV}$, while front surface protons reach a maximum energy of $4 \mathrm{MeV}$.

Our results, both experimentally and through PIC simulations, offer additional support to the back-surface electrostatic sheath described by the TNSA model presented in Chapter 3. The data presented show quite clearly that protons with energy $>5 \mathrm{MeV}$ originate predominantly from the back surface of the laser target. PIC simulations performed to model our experimental results show protons on the back surface reach a maximum energy several times higher than those on the front surface. Protons accelerated on the front surface of the laser target do not reach a maximum energy several times the ponderomotive potential, but only approximately equal to the ponderomotive potential. If the laser target was very thin (i.e. $\sim 1 \mu \mathrm{m}$ as in the case of the Michigan experiments presented in Chapter 3), a back surface sheath may not have time to form, and the majority of protons would gain energy from the ponderomotive potential on the front surface of the target. Within our experimental parameters $I \lambda^{2}>10^{19} \mathrm{~W} \mu \mathrm{m}^{2} / \mathrm{cm}^{2}$ and target thickness $\sim 15 \mu \mathrm{m}$, only the taregt normal sheath acceleration model (TNSA) - presented in Section 4.1 - accurately describes our results. 


\section{Chapter 7}

\section{Concluding Remarks and}

\section{Recommendations for Future Work}

Hydrogenous contaminants exist on the surface of laser targets due to hydrocarbons (from h'andling or vacuum pump oil) and water vapor present in the vacuum chamber. Irradiating a target with an ultra-intense laser pulse can accelerate these contaminants to energies of several or tens of MeV. Protons, having a high chargeto-mass ratio of $q / m=1$, are preferentially accelerated. Protons at the laser-plasma interface on the front surface of the target can be accelerated up to energies of order the ponderomotive potential of the laser pulse. At the back surface of the target, an electrostatic sheath of hot electrons can accelerate protons to energies of several times the ponderomotive potential of the laser pulse. Which of these mechanisms was the dominating process of proton acceleration, and to what extent heavier ions - such as 
deuterons - can be accelerated, were questions posed at the beginning of this thesis. In this, the final chapter of the thesis, how well these questions were answered is discussed, and recommendations are made for answering remaining questions related to laser-ion acceleration and its implications for the fast ignitor approach to ICF.

\subsection{Deuteron Acceleration and the Production of DT-Fusion Neutrons}

Removing hydrogenous contaminants from laser targets by radiative heating allowed for the acceleration of hydrogen or deuterium dopants from the interior of the target. Detection of ${ }^{196} \mathrm{Au}$ in gold samples placed near a tritiated-titanium target bombarded with laser-accelerated deuterons showed evidence of $14.1 \mathrm{MeV}$ neutrons from the DT-fusion reaction. While this result can be considered a modest success, the experiment did have several problems that need to be addressed.

Firstly, radiative heating was not the best choice for removing contamination from the target surface. Radiatively heating a target raises the temperature of the entire target, driving away contaminants from all surfaces. This makes the question of whether energetic protons originate from the front or back surface impossible to address. Also, as shown in the radiograph of Fig. 5.6, the heating element must be so close to the target in the vacuum chamber that it obscures the accelerated beam. This

proximity to the target requires that the filament be replaced often due to damage it 
sustains from debris associated with the exploding foil.

Most importantly, dopant acceleration from radiatively heated targets was difficult to reproduce. The maximum temperature of the target was highly dependant on the position of the filament. A subtle change in position - even several millimeters could result in temperature changes of tens of degrees. Removing contaminants while preserving enough of the dopant to be accelerated required finding the proper position for the heating element and strictly limiting the amount of time the target was heated. Both of these constraints were far too stringent to be considered for practical everyday use in any application of laser-ion beams.

Although the DT experiment had its problems it should be stated that there is no observable physics barrier to overcome. Achievable laser power continues to increase. As stated in Chapter 5, repeating the experiment on a petawatt-size laser could have significantly better results. With a laser system capable of generating proton beams with a maximum energy $>50 \mathrm{MeV}$, a deuteron beam of high yield and energy should be easy to produce after removing surface contaminants by ion sputtering. Once a deuteron beam is repeatably produced, it would be a simple matter of irradiating a tritiated target to produce fusion neutrons. A picosecond pulse of $14.1 \mathrm{MeV}$ neutrons would have beneficial applications to fast neutron diagnostics for the National Ignition Facility, neutron radiography and time-resolved neutron damage studies. 


\subsection{The Origin of Accelerated Protons}

Selectively removing contamination from one surface of the laser target - either the front or back surface - at a time allowed us to answer the question of whether energetic protons originated from the front or back surface of the target. Using an argon-ion sputter gun to remove contamination by etching the target surface, was both effective and repeatable.

As we can see from the data shown in Fig. 6.6 and the analysis shown in Fig. 6.7, etching the front surface of the laser target had no measurable affect on the proton beam, while etching the back surface of the laser target significantly reduced the total yield of protons with energies $>5 \mathrm{MeV}$. In fact, the total yield of protons was reduce to less than $1 \%$ of a typical yield when contaminants were removed from the back surface. PIC simulations specific to our experimental parameters showed that protons on the front surface of the target reach a maximum energy of approximately the laser-ponderomotive potential $(\sim 4 \mathrm{MeV})$ while those on the back surface reach a maximum energy of several times the ponderomotive potential $(\sim 13 \mathrm{MeV})$, as shown in Fig. 6.11.

\subsection{Future Work}

Now that hydrogenous contaminants can be effectively removed from targets, and the back surface sheath effect has been shown to be the dominant mechanism in laser- 
ion acceleration, the challenge remains to customize the ion beam to the necessary parameters for fast ignition. Energy deposition into a assembled fuel may be enhanced by accelerating ions heavier than protons. Targets made of materials with appropriate charge-to-mass ratios could be cleaned of contaminants and irradiated to produce heavy ion beams. High-Z elements (i.e. gold) with small charge-to-mass ratios would be mostly immobile for most of the duration of the sheath field and gain little energy. Lighter elements (i.e. carbon, fluorine) have been shown to reach energies up to $100 \mathrm{MeV}$ [48]. Targets fabricated with a graphite or light-element chemical compound layer on the back surface of the target could generate high energy beams of heavierthan-proton ions.

Focusing the ion beam to a spot size several microns in diameter may prove the highest obstacle to overcome for fast ignition. However, given the laminar nature of the expanding ion beam and the fact that the sheath field is normal to the back surface of the target, ballistically focusing the ion beam by shaping the laser target should be possible. Patel et al. have already shown that irradiating half-spherical shells increases the energy deposited in a secondary foil behind the laser target [64]. Energetic protons from a $320-\mu \mathrm{m}$ diameter hemispherical Al shell produce a heated area 16 times smaller in a $10 \mu \mathrm{m} \mathrm{Al}$ foil placed in a plane coinciding with the geometric center of the shell, than when an $\mathrm{Al}$ foil was placed a similar distance from a flat foil. A schematic of the experimental setup and significant results is reproduced in Fig. 7.1. 


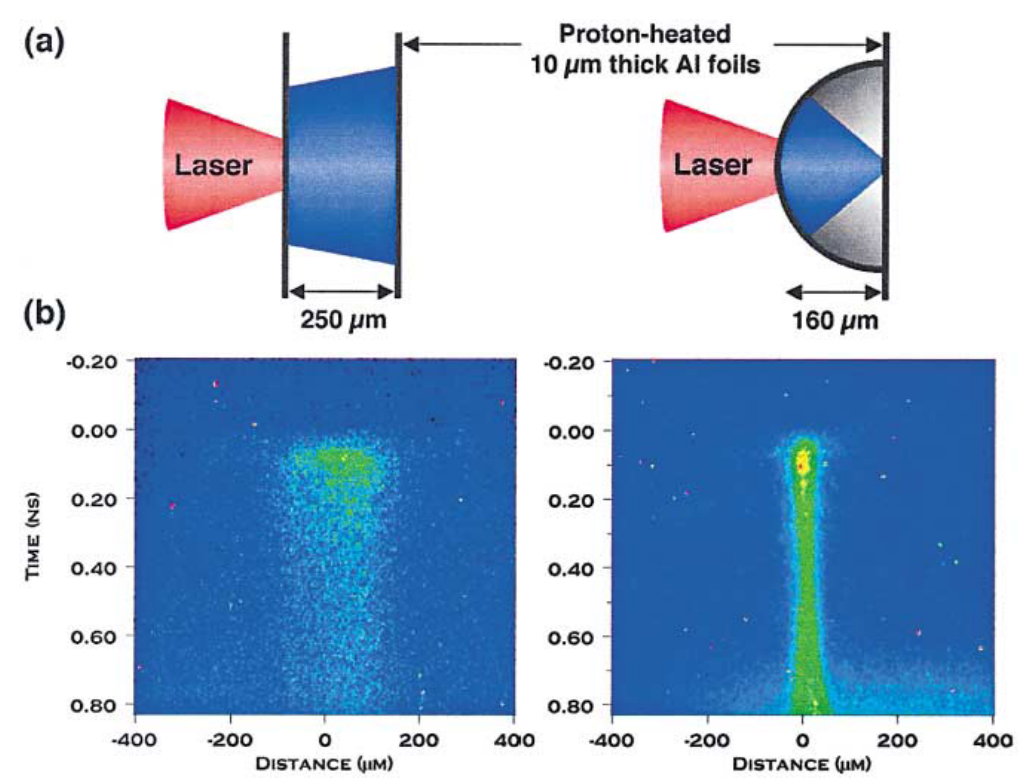

Figure 7.1: (a) Experimental setup for flat and focusing target geometries. Each target consists of a flat or hemispherical 10- $\mu \mathrm{m}$ thick Al target irradiated by the laser, and a flat $10-\mu \mathrm{m}$ thick $\mathrm{Al}$ foil to be heated by the protons. (b) Corresponding streak camera images showing space- and time-resolved thermal emission at $570 \mathrm{~nm}$ from the rear side of the proton-heated foil. The streak camera images an $800 \mu \mathrm{m}$ spatial region with a $1 \mathrm{~ns}$ temporal window. Figure used with permission from P. Patel [64].

Fast ignition experiments are currently underway at several labs around the world. The Institute of Laser Engineering (ILE) at Osaka University has a 12-beam laser facility capable of delivering approximately $10 \mathrm{~kJ}$ of energy for direct-drive isobaric compression. Recently they have completed construction on a petawatt-class laser and have already observed an increase in neutron production up to 3 orders of magnitude when using the petawatt laser in conjunction with conventional isobaric compression [65]. ILE has also begun exploring the possibility of using re-entrant cone targets to exclude the plasma blow off from one sector of the target. Such a cone would allow the ignition laser a clear, close approach to the assembled core without relying 


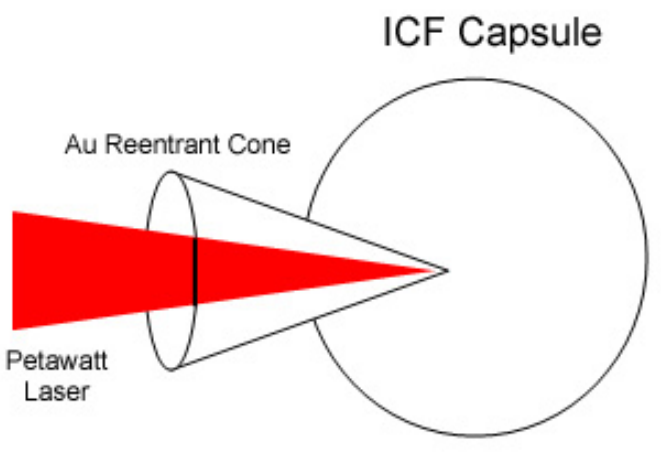

(a)

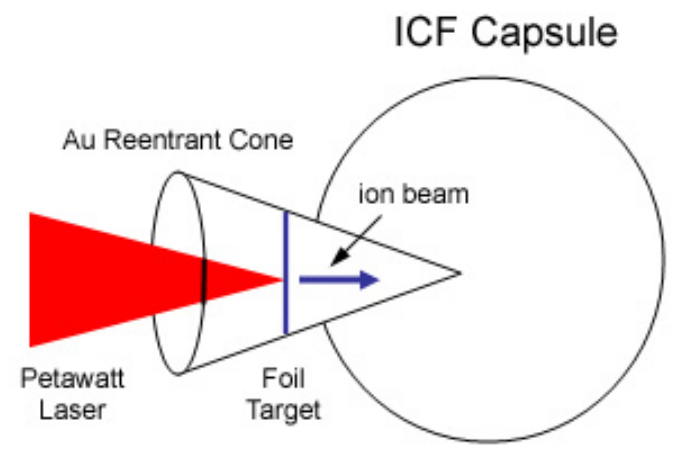

(b)

Figure 7.2: (a) Typical ICF fuel capsule shown with re-entry cone. The capsule is compressed by standard techniques then a petawatt laser enters reentrant cone to heat the assembled fuel. (b) Capsule is compressed by standard techniques, but petawatt laser enters reentrant cone to irradiate a target, producing an ion-beam to heat assembled fuel.

on ponderomotive tunneling [66]. A simple diagram of such a target is shown in Fig. 7.2(a). For a detailed review of the fast ignitor research at ILE please refer to Ref. [67].

The Laboratory for Laser Energetics (LLE) at the University of Rochester has begun construction on the OMEGA EP, a petawatt laser facility to be used in conjunction with the existing 60-beam OMEGA laser. A two-beam facility, each beam of the OMEGA EP${ }^{1}$ will have energy capability of $2.6 \mathrm{~kJ}$ per beam and pulse lengths ranging from 1 to $100 \mathrm{ps}$, capable of producing a peak power of 2 to $3 \mathrm{PW}$ and focused intensities up to $6 \times 10^{20} \mathrm{~W} / \mathrm{cm}^{2}$. The OMEGA EP will demonstrate the effectiveness of fast ignition on a compressed fusion capsule and show the feasibility of a similar short pulse system on the NIF.

\footnotetext{
${ }^{1}$ Expected laser parameters given in DOE, February 2003 Progress Report on the Laboratory for Laser Energetics, Inertial Confinement Fusion Program Activities.
} 
Although neither of the facilities mentioned above were designed to demonstrate ion-beam assisted fast ignition, the opportunity exists. Re-entrant cone targets, for example, could be modified to produce energetic ion beams by the simple inclusion of a flat (or curved) foil target within the cone. This modification is shown in Fig. 7.2(b). The petawatt laser would produce an energetic ion beam capable of depositing energy very effectively into the assembled fuel of the compressed capsule. A re-entrant cone approach to ion-assisted fast ignition may be more feasible than the modified hohlraum approach described by Roth et al. presented in Chapter 1 .

The advantages of fusion over other forms of power will ensure the continuation of ICF research around the world at various academic and government laboratories for many years to come. Until ignition is achieved in the laboratory, no path to controllable fusion can ruled out. For this reason, fast ignitor research will equally continue and, as many hope, hasten the first practical demonstration of inertial fusion power. Ion acceleration from ultra-intense laser pulses could improve the feasibility of the fast ignitor in both the direct and indirect drive approaches to ICF. 


\section{Appendix A}

\section{Adsorption of Water onto $\mathrm{Au}$ and}

\section{Al Foils}

The field of adsorption and surface science in general has been a well developed

field for over a century. The volume of information available on the subject led J. E.

Lennard-Jones, an eminent scientist in the field, in 1932 to state:

The literature pertaining to the sorption of gases by solids is now so vast that it is impossible for any, except those who are specialists in the experimental technique, rightly to appraise the work, which has been done, or to understand the main theoretical problems which require elucidation [68].

With this in mind, what follows is a nonspecialist's view of the pertinent aspects of adsorption theory that explain why contaminants are easier to remove from gold foils than aluminum foils. 
Adsorption is the physical process by which molecules are attracted to and become attached to material surfaces. This is different from absorption, in which molecules diffuse past the surface, entering the solid in much the same way a gas dissolves into a liquid. In the case of solids, a molecule (or atom) must first be adsorbed onto the surface before in can be absorbed into the bulk of the solid itself. There are two principal mechanisms for the adsorption of molecules on surfaces:

Physisorption Molecular attraction and bonds are due to weak Van der Waalstype forces. There is no significant redistribution of electron density in either the molecule or at the substrate surface. Typical energies of physisorption are between 1 and $10 \mathrm{kcal} / \mathrm{mol}$.

Chemisorption Molecular bonds are broken due to chemical reactions, involving substantial rearrangement of electron densities in the adsorbate and the substrate. Typical energies of chemisorption are between 10 and $200 \mathrm{kcal} / \mathrm{mol}$.

A major breakthrough in the understanding of adsorption was achieved when Lennard-Jones first postulated that the potential energy associated with adsorption was governed by these two processes [68]. A simply drawing similar to the one Lennard-Jones proposed is shown in Fig. A.1. As shown in the figure, chemisorption has a much deeper potential well than that of physisorption. This model is known as activated adsorption, since bonds between the atoms in the impinging gas sufficiently activate to overcome the energy barrier. 


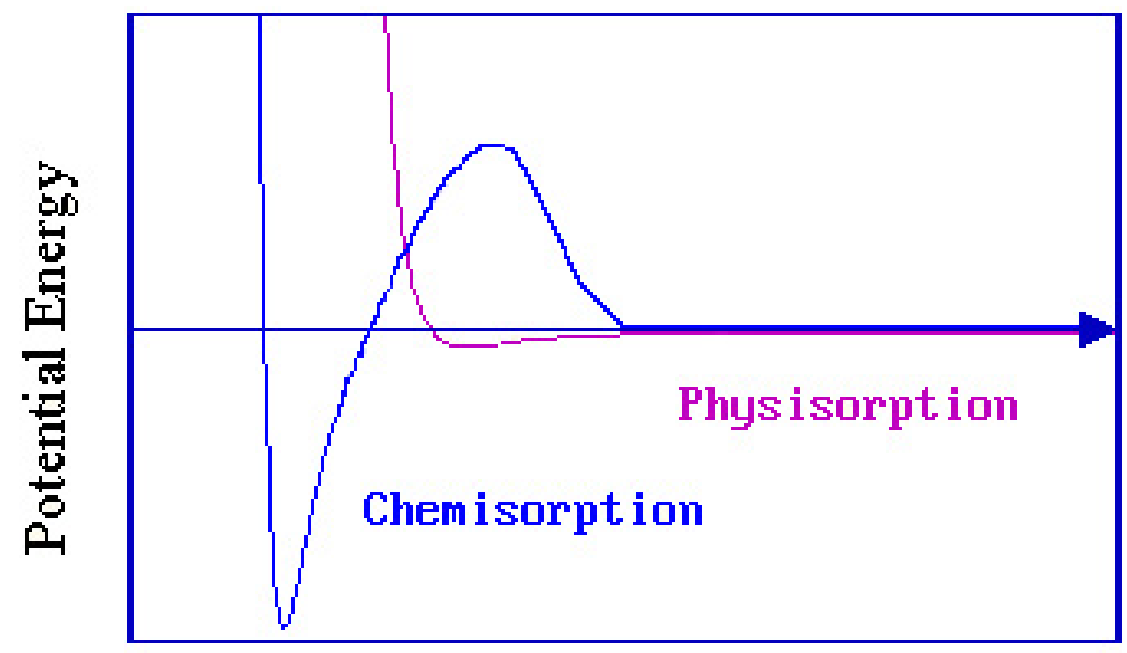

Distance from Surface

Figure A.1: Qualitative drawing of adsorption potential energy curve. The gas-surface interaction is governed by either physisorption of chemisorption.

Adsorbate layers typically have densities between those of a gas and a liquid. At liquid density, it does not take many molecules to form a monolayer. As an example, let's consider the adsorption of water in a layer $3 \AA$ thick on a surface. The coverage of adsorbed water in molecules $/ \mathrm{cm}^{2},\left[n_{A}\right]$, is given by,

$$
\begin{aligned}
{\left[n_{A}\right] } & =\left(1 \frac{\mathrm{g}}{\mathrm{cm}^{3}}\right)\left(3 \times 10^{-8} \mathrm{~cm}\right)\left(\frac{6 \times 10^{23} \text { molecule }}{18 \mathrm{~g}}\right) \\
& =1 \times 10^{15} \frac{\text { molecules }}{\mathrm{cm}^{2}} .
\end{aligned}
$$

From Eq. (A.1) we can see that a monolayer of water contains $\sim 10^{15} \frac{\text { molecules }}{\mathrm{cm}^{2}}$.

When adsorption occurs on a clean surface, heat is liberated during the formation of the surface bond. The heat of adsorption, $\Delta H_{a d s}$, associated with the layer of the adsorbate is determined by the interaction strength between atoms and molecules in 
the monolayer and the surface on which they are adsorbed (for a review of monolayer adsorption, c.f. Ref. [69]). The net rate of physisorption of a gas onto a solid surface can be written in the form

$$
F\left[\frac{\text { molecules }}{\mathrm{cm}^{2} \mathrm{~s}}\right]=\alpha k\left[n_{A}\right]_{g}-k^{\prime}\left[n_{A}\right]_{s}
$$

where $\left[n_{A}\right]_{s}\left[\frac{\text { molecules }}{\mathrm{cm}^{2}}\right]$ is the surface concentration of the adsorbate and $\left[n_{A}\right]_{g}\left[\frac{\text { molecules }}{\mathrm{cm}^{3}}\right]$ is the concentration of the species that exists in the gas phase. We can simplify Eq. (A.2) and take the rate of desorption to be zero, which leaves us with the net rate of gas molecules striking the surface,

$$
F\left[\frac{\text { molecules }}{\mathrm{cm}^{2} \mathrm{~s}}\right]=\alpha k\left[n_{A}\right]_{g}
$$

where

$$
k=\left(\frac{R T}{2 \pi M_{A}}\right)^{1 / 2}[\mathrm{~cm} / \mathrm{s}]
$$

where $\alpha$ is the sticking coefficient. $M_{A}$ is the molecular weight of the impinging molecules, $R$ is the gas constant and $T$ is the temperature in Kelvin. The surface concentration, $\left[n_{A}\right]_{s}$, under these conditions is the product of the incident flux, $F$, and the surface residence time $\tau$,

$$
\left[n_{A}\right]_{s}=\alpha F \tau
$$

The residence time in the above equation is sometimes called the sojourn time, and defined as the average time spent by an adsorbed molecule at a particular site. If the incident molecule remains on the surface long enough to achieve thermal equilibrium 
with the surface atoms, $\tau$ has the form,

$$
\tau=\tau_{o} e^{\Delta H_{a d s} / R T},
$$

where $\tau_{o}$ (typically with a value between $10^{-12} \mathrm{~s}$ and $10^{-13} \mathrm{~s}$ ) is associated with the average vibrational frequency of the immobile adsorbate. The heat of adsorption is defined as the binding energy of the adsorbed species and is always positive. Clearly, the higher $\Delta H_{a d s}$ and the lower the temperature, $T$, the longer the residence time.

If we substitute the vapor density by the pressure using the ideal gas law $\left[n_{A}\right]_{g}=$ $N_{A} P / R T$ (where $N_{A}$ is Avogadro's number) we can rewrite Eq. (A.5) as:

$$
\left[n_{A}\right]_{s}=\frac{\alpha P N_{A}}{\sqrt{2 \pi M_{A} R T}} \tau_{o} e^{\Delta H_{a d s} / R T} .
$$

We can further simplify this by taking $\alpha \approx 1, R=8.3144 \times 10^{7}\left[\mathrm{erg} \mathrm{mol}^{-1} \mathrm{~K}^{-1}\right]$, and making use of the identity 10 grams $/\left(\mathrm{s}^{2} \mathrm{~cm}\right)=.0075$ torr rewrite Eq. (A.7) as

$$
\left[n_{A}\right]_{s}=3.52 \times 10^{22} \frac{P_{\text {torr }}}{\sqrt{M_{A} T}} \tau_{o} e^{\Delta H_{a d s} / R T},
$$

where the pressure is in [torr].

There is a shortcoming to Eq. (A.8) that needs correcting. We have not accounted for the fact that monolayer formation can only occur while sites for the adsorbate are unoccupied. If there is no room for a gas molecule to adsorb onto the surface, monolayer formation must stop. Langmuir developed a useful model for describing this process [70]. He assumed that any gas molecule that strikes an adsorbed molecule must be reflected, while any molecule that strikes the bare surface must be adsorbed. 
If $\left[n_{o}\right]$ is the surface concentration on a completely filled surface, the concentration of sites available for adsorption after building up an adsorbate concentration $\left[n_{A}\right]_{S}$ is $\left[n_{o}\right]-\left[n_{A}\right]_{S}$. Of the flux, $F$, striking the surface, a fraction $\left[n_{A}\right]_{S} /\left[n_{o}\right]$ will strike molecules already adsorbed and therefore be reflected. Thus, only the fraction (1$\left[n_{A}\right]_{S} /\left[n_{o}\right]$ ) of the total flux will be available for adsorption. As a result Eq. (A.5) must be modified to

$$
\left[n_{A}\right]_{S}=\left(1-\frac{\left[n_{A}\right]_{S}}{\left[n_{o}\right]}\right) F \tau
$$

which modifies Eq. (A.8) to:

$$
\left[n_{A}\right]_{s}=\frac{3.52 \times 10^{22} \frac{P_{\text {torr }}}{\sqrt{M_{A} T}} \tau_{o} e^{\Delta H_{\text {ads }} / R T}}{1+\frac{1}{\left[n_{o}\right]} 3.52 \times 10^{22} \frac{P_{\text {torr }}}{\sqrt{M_{A} T}} \tau_{o} e^{\Delta H_{\text {ads }} / R T}} .
$$

We can see from Eq. (A.10) for a given adsorption energy, the concentration of the adsorbate on the surface will increase with increasing pressure and decrease with increasing temperature. For a weakly reacting adsorbant, only capable of physisorption (such as water vapor on gold, $\Delta H_{a d s}=3 \mathrm{kcal} / \mathrm{mol}$ [71]), we can see that monolayers are difficult to form in regions of high vacuum and moderate temperatures. From a three dimensional plot of the surface concentration function, shown as Fig. A.2 we can see that even at low temperatures an adsorbant cannot remained physisorbed to the surface at low pressures. At standard operating pressure, $10^{-5}$ torr, and temperature, $300^{\circ} \mathrm{K}$, Eq. (A.10) predicts a surface concentration of $\left[n_{A}\right]_{s}=7.34 \times 10^{5}$ molecules $/ \mathrm{cm}^{2}$. This is more than nine orders of magnitude away from a monolayer concentration of $10^{15}$ molecules $/ \mathrm{cm}^{2}$. 


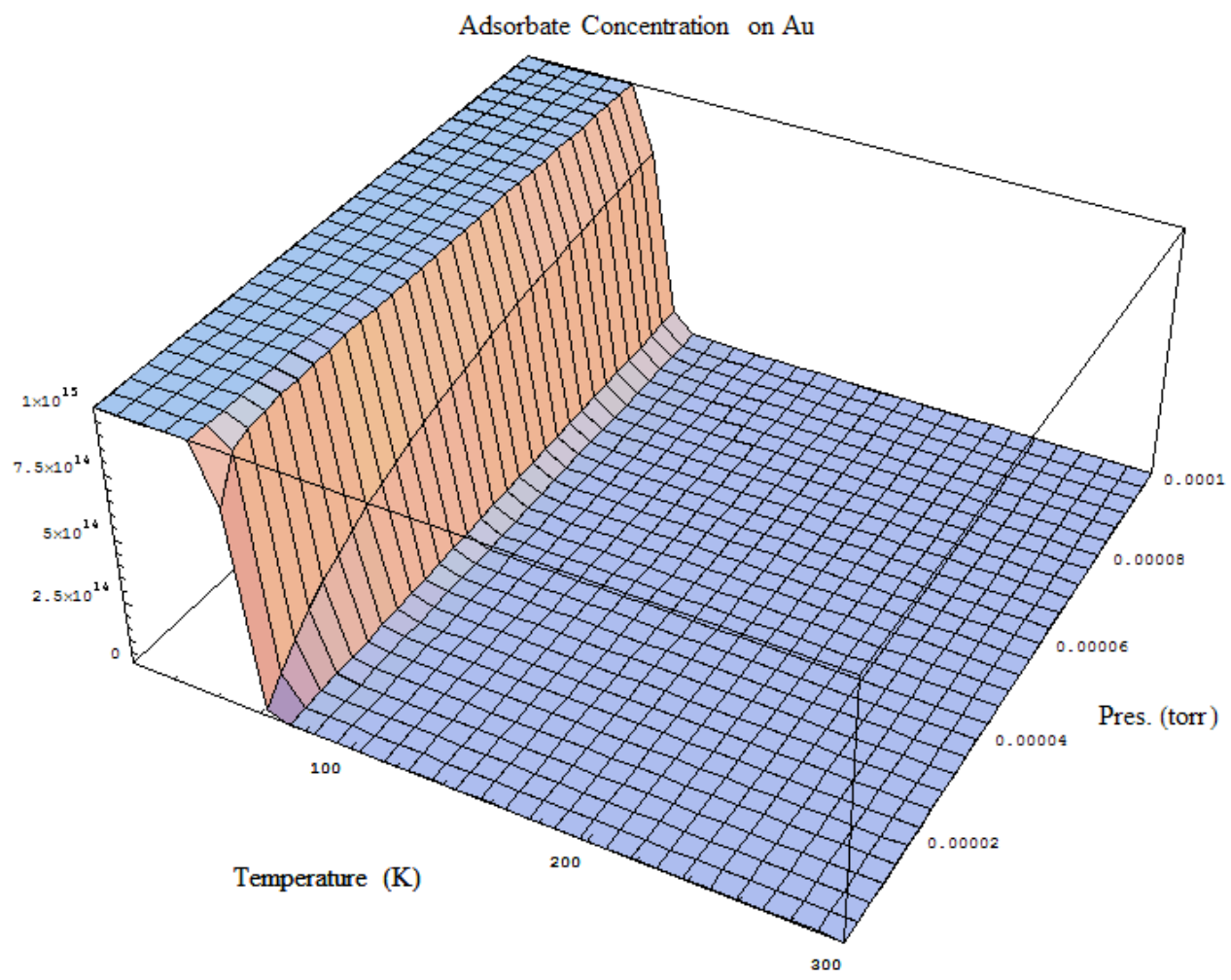

Figure A.2: Three dimensional plot of the adsorbate concentration of water vapor on gold. Heat of adsorption and maximum number of adsorption sites were take to be $\Delta H_{a d s}=$ $3 \mathrm{kcal} / \mathrm{mol}$ and $\left[n_{o}\right]=10^{15}$ molecules $/ \mathrm{cm}^{2}$ respectively.

However, molecules with a strong permanent dipole, such as water vapor, can react strongly with an ionic surface, such as aluminum-oxide $\left(\Delta H_{a d s} \approx 15 \mathrm{kcal} / \mathrm{mol}\right)$ [72]. If an oxide layer is present on the aluminum a monolayer can form very easily at room temperature even at low pressures. Fig. A.3 shows a three dimensional plot of adsorbate concentration at this higher adsorption energy. We can see from the plot that a monolayer forms very easily at standard operating pressure and temperature. Eq. (A.10) predicts a surface concentration of $\left[n_{A}\right]_{s}=4.1 \times 10^{14}$ molecules $/ \mathrm{cm}^{2}$ less than one order of magnitude away from a completely covered surface. 


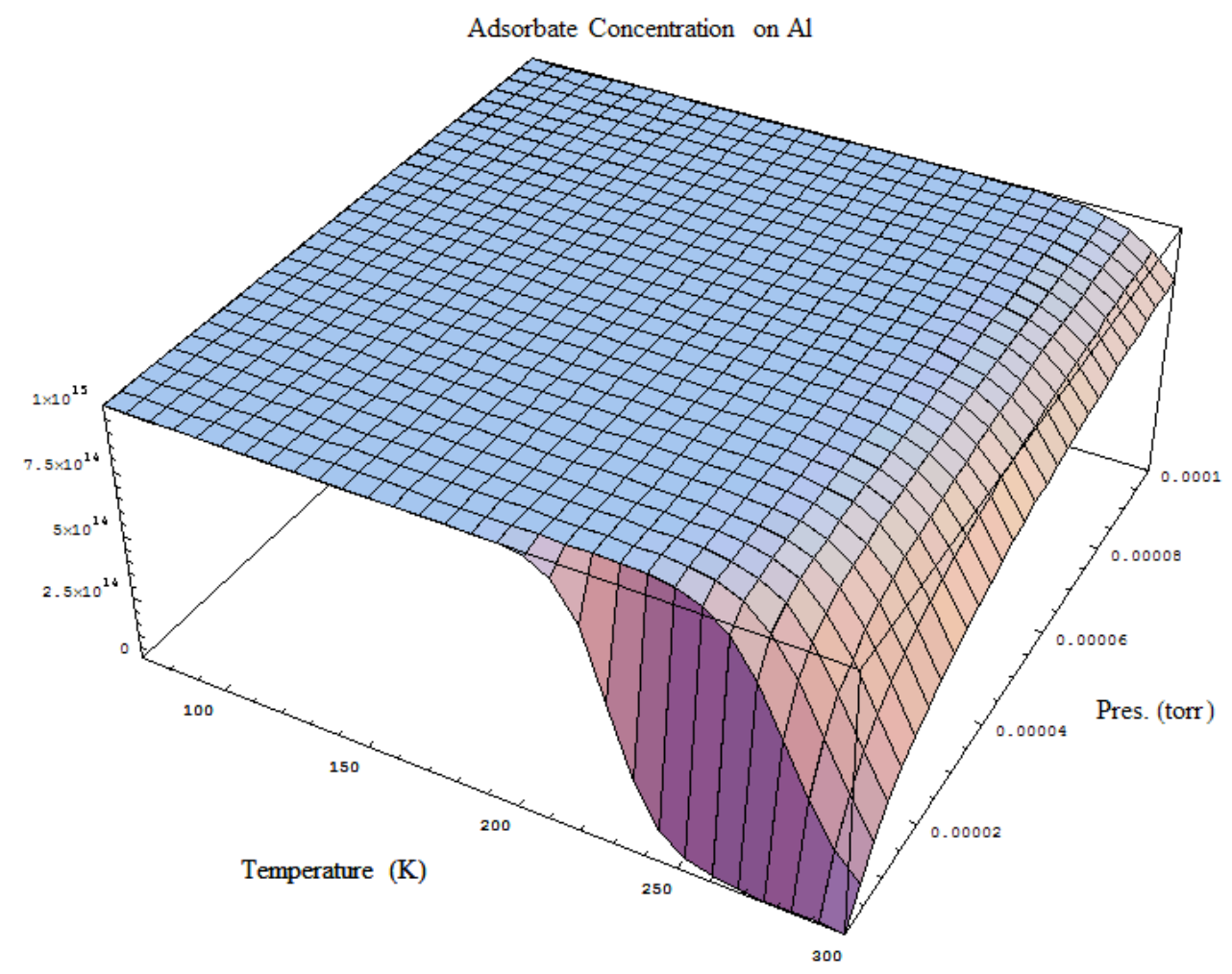

Figure A.3: Three dimensional plot of the adsorbate concentration of water vapor on aluminum-oxide. Heat of adsorption and maximum number of adsorption sites were take to be $\Delta H_{a d s}=15 \mathrm{kcal} / \mathrm{mol}$ and $\left[n_{o}\right]=10^{15}$ molecules $/ \mathrm{cm}^{2}$ respectively.

The above description of surface adsorption helps to explain why gold targets are much easier to clean by ion sputtering than aluminum targets. Given that the primary source of contamination in the target chamber is water vapor, once the ever-present layer of hydrocarbons in removed from the surface of gold targets, the low goldwater-vapor adsorption energy will prevent re-deposition of contamination onto the target surface at operating temperature and pressure. Aluminum targets, however, have a thick oxide layer in addition to the hydrocarbon layer. This oxide layer has an adsorption energy five times larger than gold with respect to water vapor, and 
Adsorption of Water onto $\mathrm{Au}$ and $\mathrm{Al}$ Foils

monolayer formation occurs on the surface faster than ion sputtering can be effective. 


\section{Appendix B}

\section{Code: Analysis of RCF}

What follows is the source code used in the analysis of RCF presented in this thesis. Image analysis (calculation of deposited dose in the film) was performed exclusively in Mathematica, while number crunching of matrices in regard to proton stopping in the associated layers of the RCF was performed via calls to Java methods. This allowed the ease of input parameter manipulation, and real time plotting of results without having to frequently recompile the code. Both forms of the code are well commented and should be easy to follow, presuming a working knowledge of Mathematica v. 4.2 and Javav. 2.0.

Although, theoretically, Mathematica notebooks can be saved as $\mathrm{T}_{\mathrm{EX}}$ files, getting one's version of $\mathrm{T}_{\mathrm{E}} \mathrm{X}$ to recognize the specific fonts and packages necessary to read such a file is rather agonizing. Therefore the presentation of the Mathematica code may have one or two errors that escaped detection during proof reading. Please 
Code: Analysis of $R C F$

accept my apologies should such errors be discovered. The Java code presented is exactly like the source thanks to the very useful \verbatim environment distributed with most forms of $\mathrm{HT}_{\mathrm{E}} \mathrm{X} 2_{\varepsilon}$.

\section{B.1 Mathematica Script}

\section{- Loading the Image}

The very first thing we have to do is load the necessary packages.

$$
\begin{aligned}
\text { In[1]: }= & \text { Clear ["Global'*"]; } \\
& \text { Needs ["Graphics 'Graphics'"] } \\
& \text { Needs ["Graphics'Colors'"] } \\
& \text { Needs ["Graphics 'MultipleListPlot'"] }
\end{aligned}
$$

Next, we need to define the file path and import the image. We start by importing the Unsigned 16-bit bytes from the LLNL specific .img file. Remember the .img file is generated in little Endian byte order, so we must specify that (ByteOrdering $\rightarrow-1$ ) in the import command.

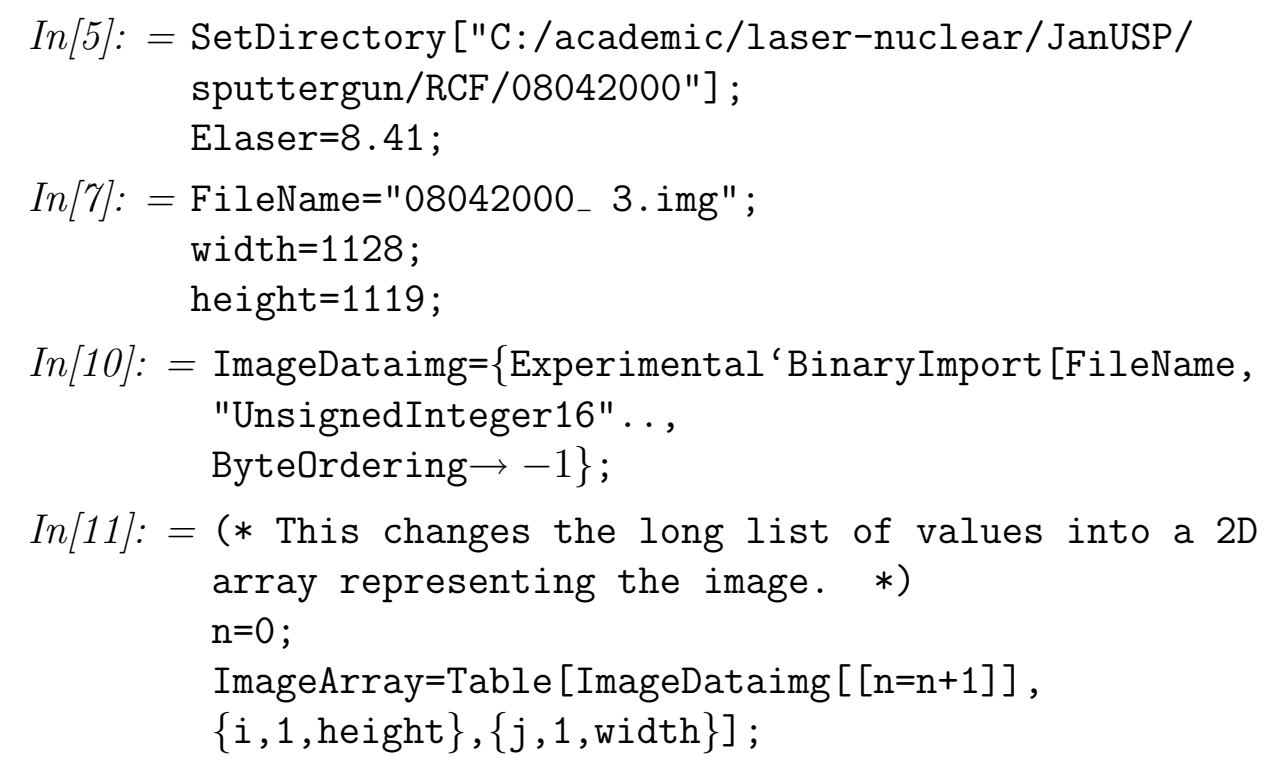

- Displaying the Image 
To display the image, we simply use the ListDensityPlot function, which plots a $2 \mathrm{D}$ array of various height values.

$$
\text { In[13]: = FullImage=ListDensityPlot [ImageArray, Mesh } \rightarrow \text { False }] \text {; }
$$

\section{- Region-Of-Interest Analysis}

Here we select the region of interest by defining two corner points of a square. The region of interest should be selected to surround the entire proton image and then remain the same for each piece of film for a particular shot. There is one subtlety, in that the index for the ROI is defined in terms of $\{\{\mathrm{y} 1, \mathrm{x} 1\},\{\mathrm{y} 2, \mathrm{x} 2\}\}$.

$$
\begin{aligned}
& \operatorname{In}[14]:=\operatorname{roi}=\{\{100,100\},\{1000,1100\}\} \\
& \operatorname{In}[15]:=\text { roiImageArray=Table }[\operatorname{ImageArray}[[i, j]], \\
& \{i, \operatorname{roi}[[1,1]], \operatorname{roi}[[2,1]]\} \text {, } \\
& \{j, r o i[[1,2]], r o i[[2,2]]\}] \text {; } \\
& \text { In[16]: = FullImage }= \\
& \text { ListDensityPlot [ImageArray, Mesh } \rightarrow \text { False, } \\
& \text { PlotLabel } \rightarrow \text { FontForm ["Full Image", \{"Times-Italic",14\}], } \\
& \text { DisplayFunction } \rightarrow \text { Identity] ; roi Image }= \\
& \text { ListDensityPlot [roiImageArray, Mesh } \rightarrow \text { False, } \\
& \text { PlotLabel } \rightarrow \text { FontForm ["Selected ROI", \{"Times-Italic", 14\}], } \\
& \text { AspectRatio } \rightarrow \text { Automatic, DisplayFunction } \rightarrow \text { Identity] ; } \\
& \text { Show [GraphicsArray [\{FullImage, roi Image }\} \text {, ImageSize } \rightarrow 500 \text {, } \\
& \text { DisplayFunction } \rightarrow \text { \$DisplayFunction] ] ; }
\end{aligned}
$$

A horizontal line-out of the ROI can be generated by averaging the y-pixel values. The result is then a simple list of $\mathrm{x}$ and $\mathrm{y}$ coordinates that can be plotted with ListPlot [ ]. Ploting the line-out of the average ROI is done to determine the background level of the film. This number is then subtracted from the data. Although the conversion from O.D. to dose is very nonlinear, the background can be as high as $20 \%$ for film with low dose.

$$
\begin{aligned}
\operatorname{In}[18]:= & \text { xmaxroi=Dimensions [roiImageArray] [[1]]; } \\
& \text { ymaxroi=Dimensions [roiImageArray }[[2]] ;
\end{aligned}
$$




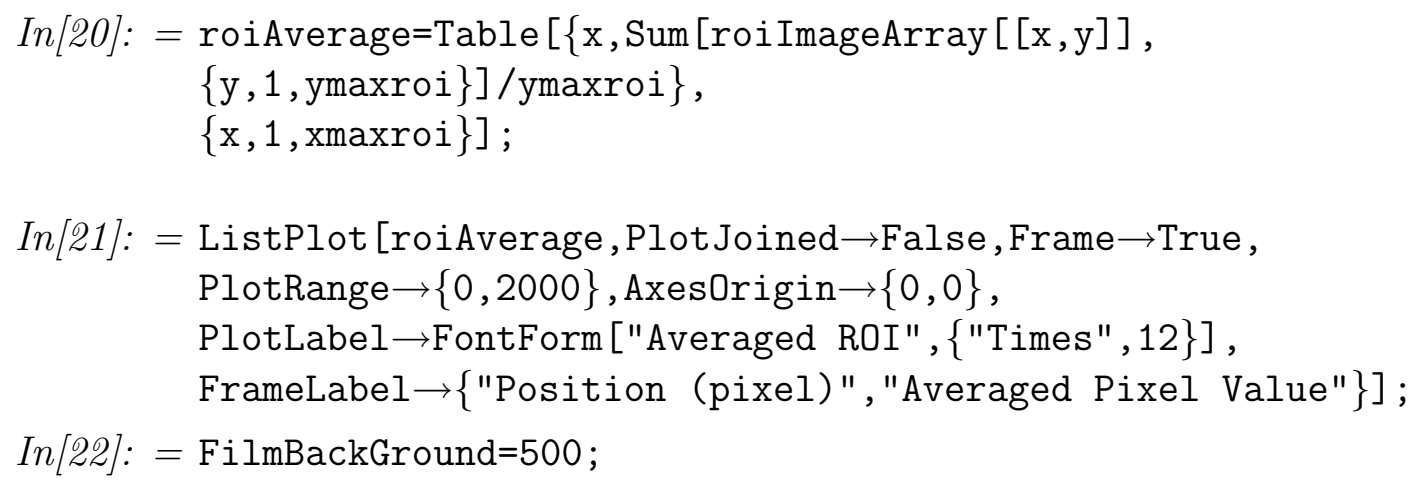

\section{- Integration Over Surface of ROI in Dose (krad)}

For the purpose of Dose calculations from radiochromic film, we are interested in the total optical density of the film. Initially, we extract a list of pixel values from the selected ROI. These are then converted to optical density (O.D.) by multiplying the pixel value by the constant, 0.00125 ,

$$
\text { O.D. }=\text { pixel value } \times 0.00125 \text {. }
$$

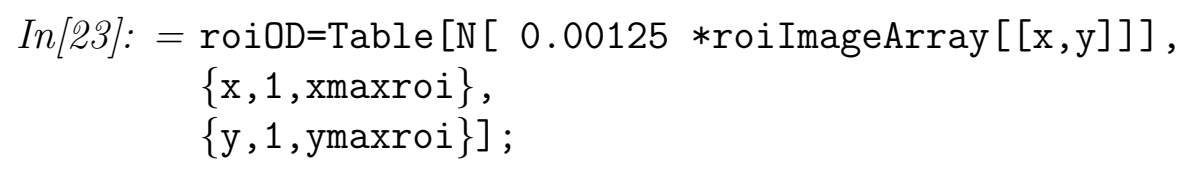

Next, we convert the optical density values into Dose given by the LLNL calibration,

$$
\begin{aligned}
\text { Dose (O. D. }) & =1.12 \times 10^{\alpha-\beta} \\
\alpha & =30.517 \text { O.D. }{ }^{0.5}+13.668 \text { O.D. }{ }^{1.3} \\
\beta & =10.8379 \text { O.D. }{ }^{0.1}+30.86 \text { O.D. }{ }^{0.9}+2.09 \text { O.D. } .^{1.7}
\end{aligned}
$$

where O.D. is the optical density and Dose is in units of krad.

$$
\begin{aligned}
\operatorname{In}[24]:= & \text { Dosekrad }\left[\mathrm{x}_{-}\right]= \\
& 1.12 * 10^{\wedge}\left(\left(-10.8379 *\left(\mathrm{x}^{\wedge} 0.1\right)\right)+\left(30.517 *\left(\mathrm{x}^{\wedge} 0.5\right)\right)\right. \\
& -\left(30.86 *\left(\mathrm{x}^{\wedge} 0.9\right)\right)+(13.668 * \\
& \left.\left.\left(\mathrm{x}^{\wedge} 1.3\right)\right)-\left(2.09 *\left(\mathrm{x}^{\wedge} 1.7\right)\right)\right)
\end{aligned}
$$

Now we simply convert the region of interest into dose. 


$$
\begin{array}{ll}
\text { MD-55 } & : \text { RCFthickness }=32 \times 10^{-4} \text { Centimeter } \\
\text { HS } & : \text { RCFthickness }=40 \times 10^{-4} \text { Centimeter } \\
\text { HD-810 } & : \text { RCFthickness }=6.5 \times 10^{-4} \text { Centimeter }
\end{array}
$$

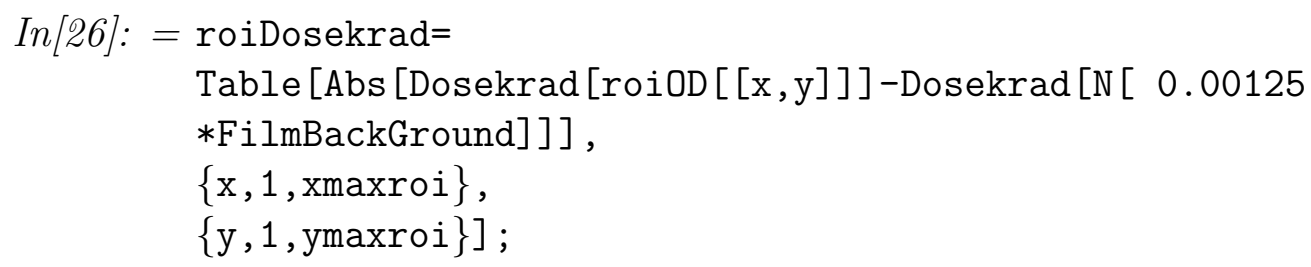

The integration under this surface gives the total dose in krads for the area contained within the selected ROI. Computationally, we equate the surface integral with a sumation of the dose values times the incremental area, which is the pixel size in $\mathrm{cm}$.

$$
\begin{aligned}
& \text { Total Dose }=\int \text { Dose } d A=\sum(\text { Dose value } \times \text { pixel area }) \\
\text { In[27]: }= & \text { pixelsize }=22 \text { 10^ }(-4) \text { Centimeter } * 22 \text { 10^ }(-4) \text { Centimeter } \\
& \left(* \text { pixel size in } \mathrm{cm}^{\wedge} 2,\right. \text { presuming a } \\
& 22-\text { micron-square pixel } *) \\
\text { In[28]: }= & \text { TotalDose=Sum[roiDosekrad }[\mathrm{x}, \mathrm{y}]] * \text { pixelsize }, \\
& \{\mathrm{x}, 1, \text { Dimensions [roiOD] }[[1]]\} \\
& \{\mathrm{y}, 1, \text { Dimensions [roiOD] }[[2]]\}] * \text { Kilorad }
\end{aligned}
$$

\section{- Total Energy Deposited}

The total dose is in units of krad $\mathrm{cm}^{2}$. However, we are after the total deposited energy described by,

$$
\text { Total Energy Deposited }=\text { Total Dose } \times \rho \times t,
$$

where Total Dose is in units of $\mathrm{J} \mathrm{\textrm {cm } ^ { 2 }} / \mathrm{g}(1 \mathrm{krad}=0.01 \mathrm{~J} / \mathrm{g}), \rho$ is the density of the $\mathrm{RCF}$ active dye layer, and $t$ is the layer thickness.

Next we simply define the remaining constant values (the SI conversion for kilorad, the RCF dye density and thickness) and solve for the energy deposited. Keep in mind, the RCF dye density is different for various film types:

$$
\begin{aligned}
\text { In[29]: }= & \text { Kilorad }=.01 \text { Joule } / \text { Gram; } \\
& \text { RCFdensity }=1.30 \text { Gram } / \text { Centimeter }^{3} ; \\
& (* \text { c.f. NIM A302, 165-176 }(1991) *) \\
& \text { RCFthickness }=4010=4 \text { Centimeter; }
\end{aligned}
$$




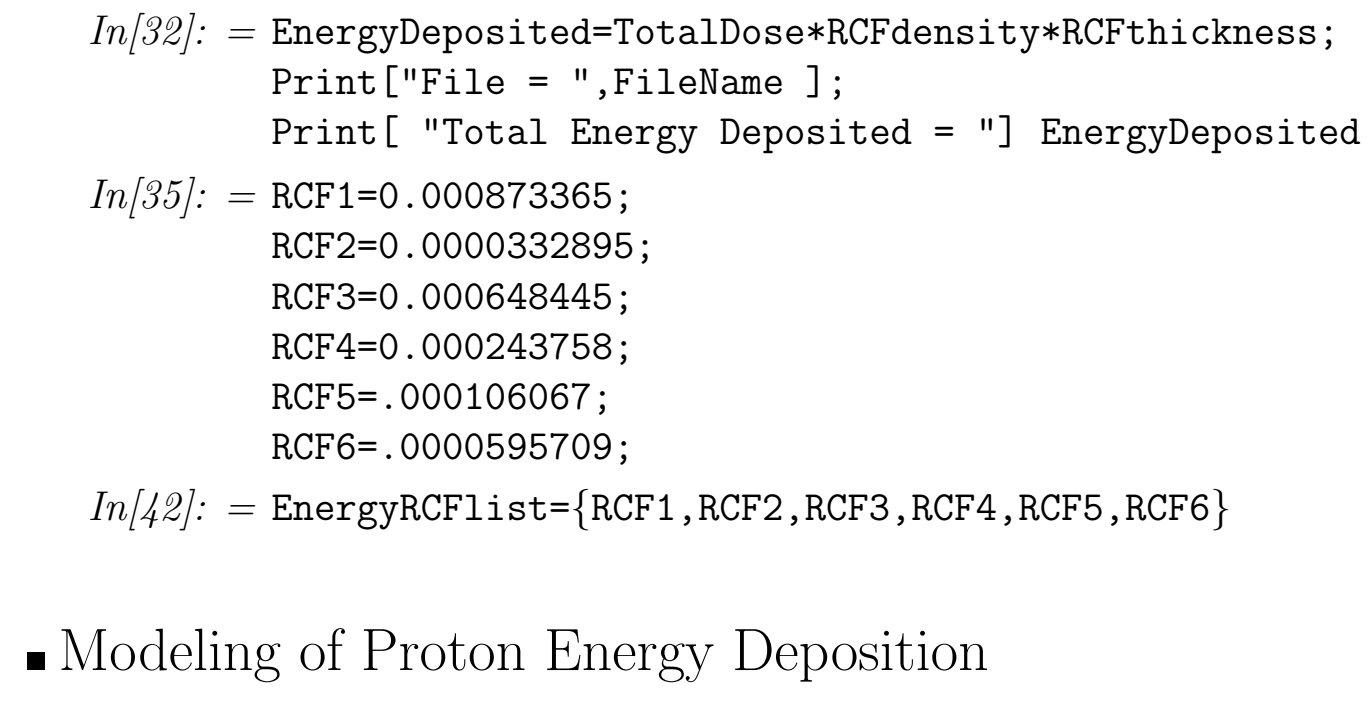

\section{- Modeling of Proton Energy Deposition}

Here, we model the energy deposited in an RCF film pack with an aluminum blast shield. This is done by using the same energy deposition technique that SRIM employs (ref: Stopping Powers and Ranges of Protons and Alpha Particles. Report No. 49, International Commission on Radiation Units and Measurements, Bethesda, MD, 1993; J.F. Ziegler, Comments on ICRU Report 49, Radiat. Res. 152, 219-222, 1999; Comments on those comments, Radiat. Res. 155, 378-381, 2001).

Due to the many nested For-loops and intense number crunching, this part of the code is done primarily with calls to Java methods. For a description of the Java code please refer to that source in class ProtonStopping.java. Several methods are used. The first creates a film pack of appropriate material and thickness. Each micron thickness of the film pack is considered one layer that the proton propagates through. The next method calculates home much energy is lost in each layer given an initial proton energy. Then each initial proton energy is normalized to a Maxwellian distribution of the form:

$$
d n=\frac{2}{\sqrt{\pi}(k T)^{3 / 2}} \sqrt{E} e^{-E / k T} d E .
$$

These energies are then sumed over each layer and the result is a Mathematica list of energy deposited in each layer of the film pack in units of $\mathrm{MeV}$. To get an accurate model to compare with ROI analysis from the film, the dye layers of the film pack must be extracted to represent each piece of RCF.

$$
\begin{aligned}
\text { In[43]: }= & \text { Needs ["JLink'"]; } \\
& \text { InstallJava[]; }
\end{aligned}
$$

Now we define the several parameters the Java code needs for the constructor. 


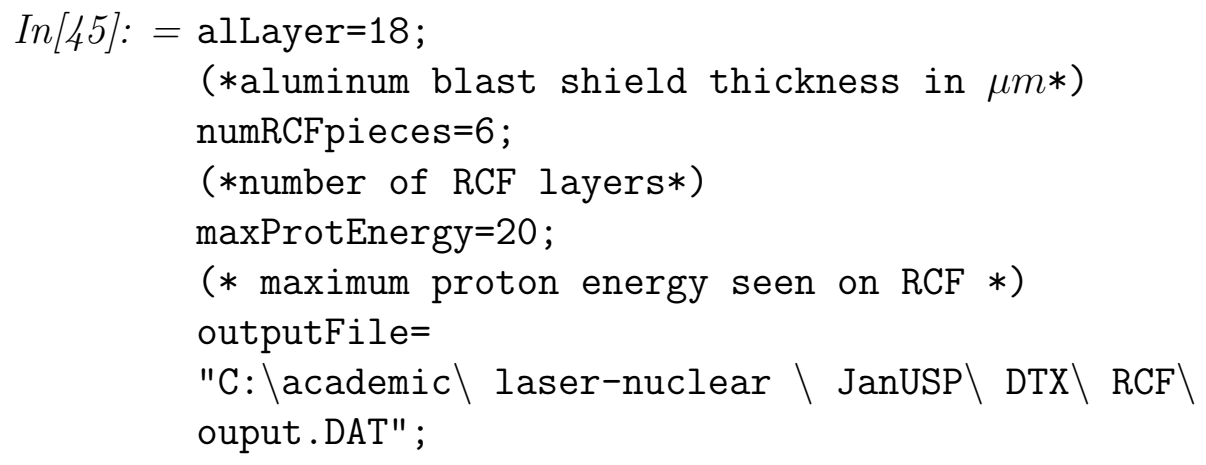

Now we simply load the class with the proper arguments for the constructor:

$$
\begin{aligned}
\text { In[49]: }= & \text { ProtStop }= \\
& \text { JavaNew ["mallen.physics.ProtonStopping", alLayer, numRCFpieces, } \\
& \text { maxProtEnergy, outputFile]; }
\end{aligned}
$$

At this point, we need to define each layer of film according to type. As stated in the Java source code, the definitions are as follows: $\mathrm{HS}=1, \mathrm{MD}-55=2$, HD$810=3$. For example, a film pack consiting of two fronts layers of HD-810 and the next four layers of HS would be represented as: ProtStop@numRCF $=\{3,3,1,1,1,1\}$. SetfilmPackSize is then called to create an empty array of the proper number of 1 micron layers.

$$
\begin{aligned}
\operatorname{In}[50]:= & \operatorname{numRCF}=\{3,3,1,1,1,1\} ; \\
& \text { ProtStop@numRCF }=\text { numRCF } ; \\
& \text { ProtStop@setFilmPackSize []; }
\end{aligned}
$$

Call methods to perform peicewise calculation:

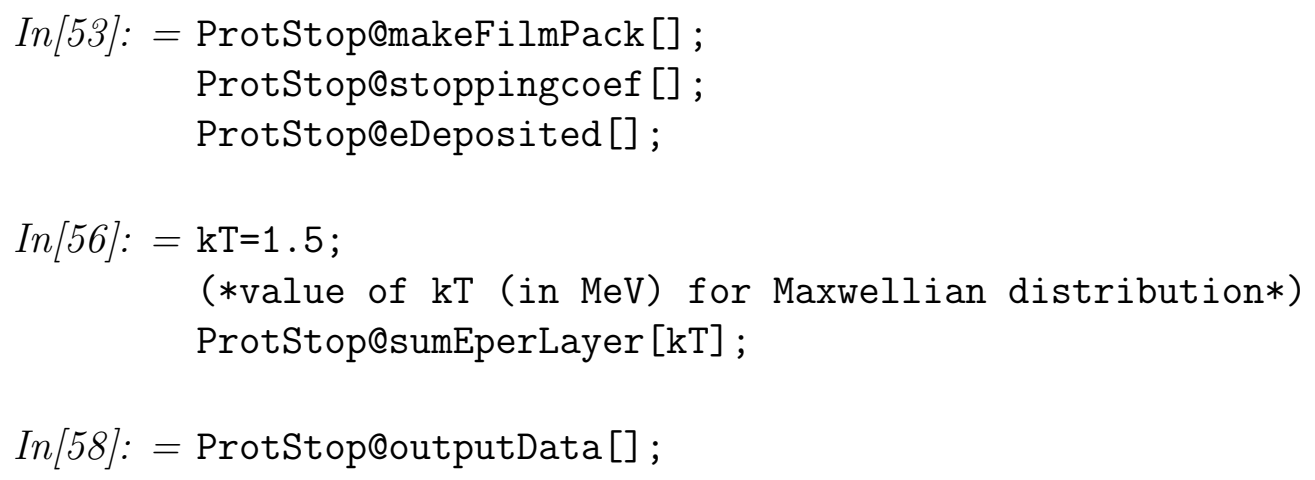

Now grab the final Energy-deposited-per-Layer list from the Java class:

$$
\text { In[59]: = EperLayer=ProtStop@totalEperLayer; }
$$


All we do now is extract only the dye layers and sum them over each piece of RCF. Remeber, the term EnergyRCFList \{\} has been defined above for the ROI film analysis, along with variable RCF1 through RCF-N.

This part is rather complicated. There is probably an easier of way of doing it, but to date I haven't found one. First we start by extracting all of the values contained only in the dye layers. This is done by comparing the EperLayer array with the filmPack array. The array TotalDyeRCF contains only the values from EperLayer of the dye layers.

$$
\begin{aligned}
\text { In[60]: }= & \text { filmPackLayer }=\text { ProtStop@filmPack; } \\
& \text { TotalDyeRCF }=\{\} ; \\
\text { In[62]: }= & \text { Do }[ \\
& \text { If }[\text { filmPackLayer }[[i]]==94, \\
& \text { TotalDyeRCF }=\{\text { TotalDyeRCF, EperLayer }[[i]]\} / / \text { Flatten }], \\
& \{\text { i, } 1, \text { Length }[\text { filmPackLayer }]\}] ;
\end{aligned}
$$

Now that we have an array with just the Energy deposited in the dye layers, we must 1. group the values according to each layer of film and then 2 . sum the values to get a total value deposited in each piece of film. The total energy deposited in each piece of RCF for the fit is referred to as RCFpiece[N].

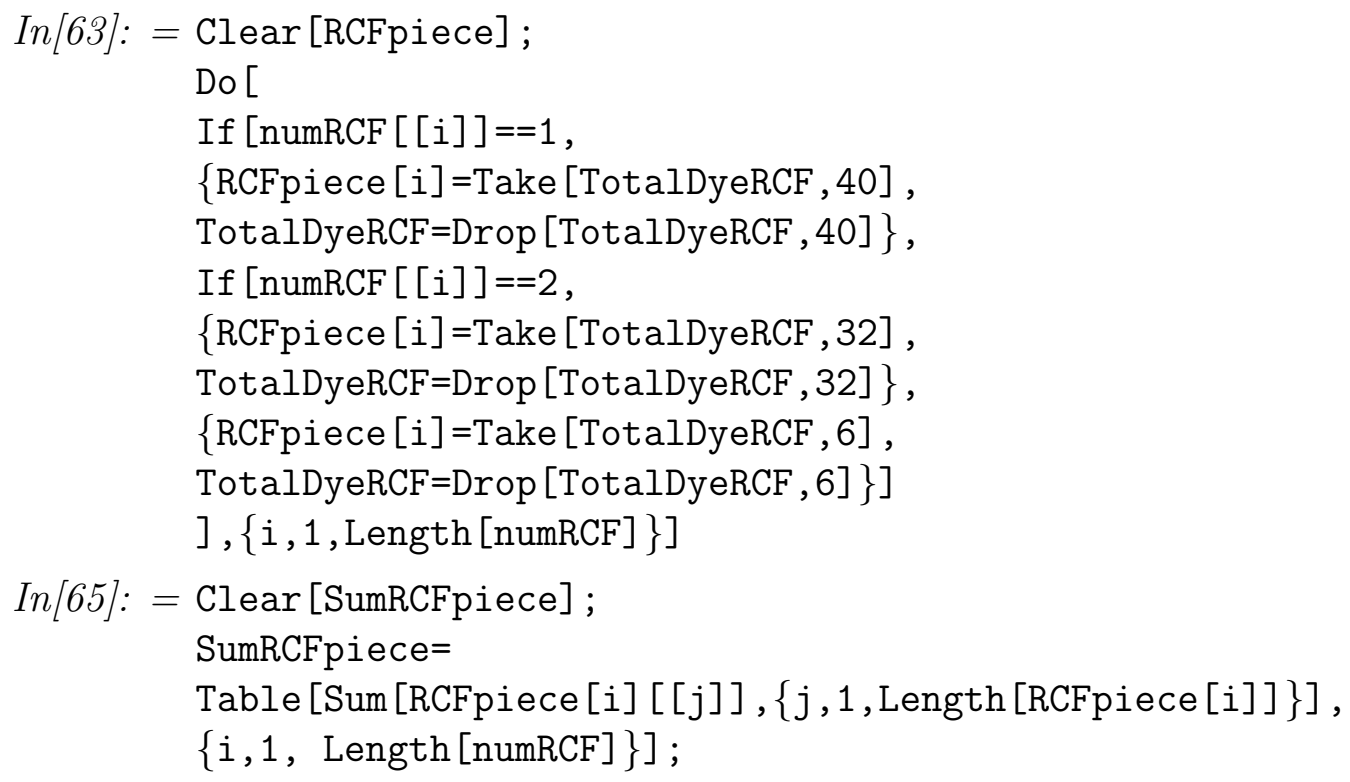

We now have the total energy (calcualted total energy) deposited in each piece of RCF. This information is held in array SumRCFpiece. Now all we do is adjust the scaling parameters until the measured data points fit with the calculated points.

$$
\operatorname{In}[67]:=\text { Nyield=1.5 10^11; }
$$




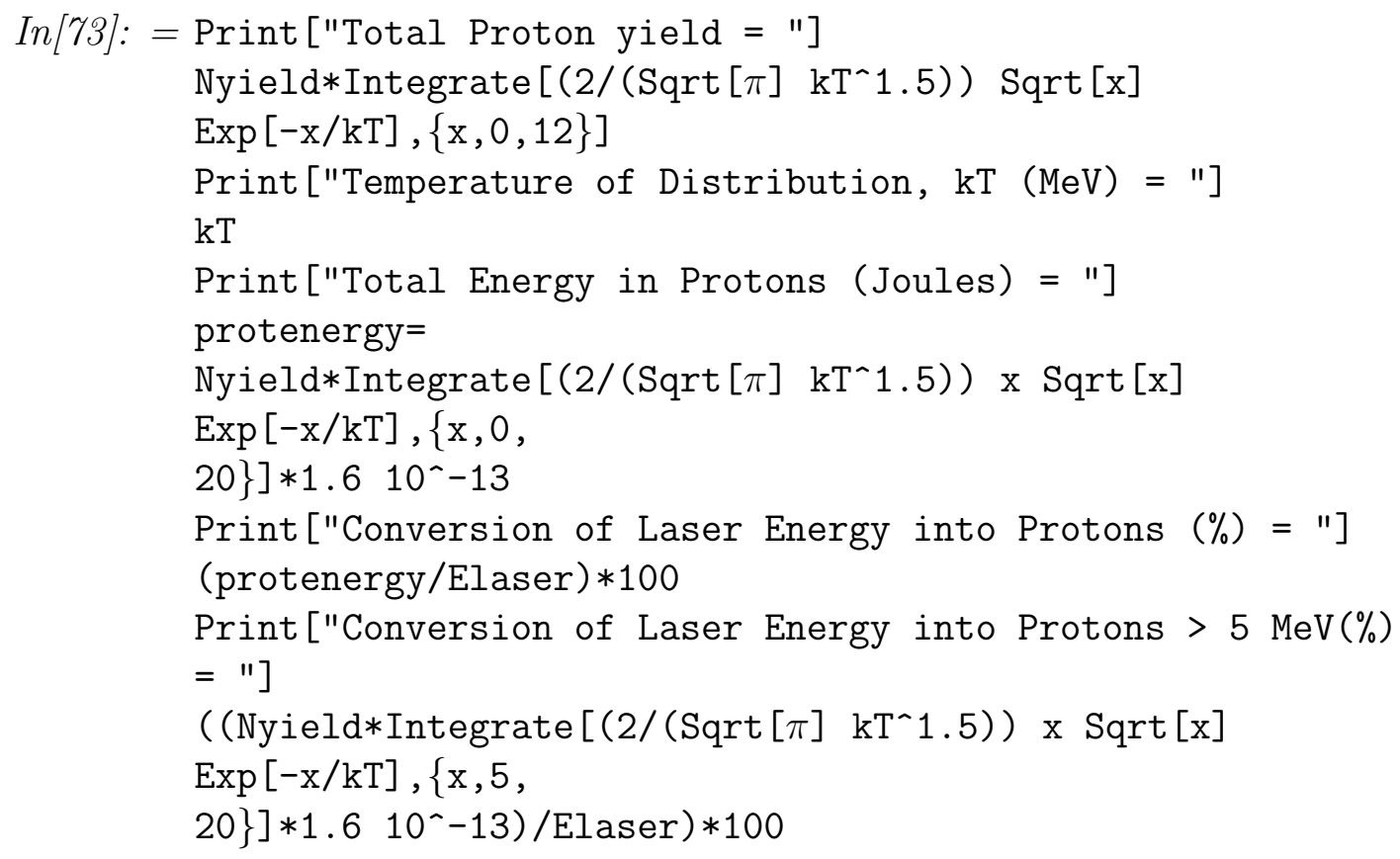




\section{B.2 Java Script}

/*

Purpose:

Generate a 2-D spreadsheet of proton energy deposited in each succesive layer of a film pack. Calculate (Sum) energy deposited in each dye layer of RCF.

1. setVariables(): : initializes some class wide variables: int alThickness (thickness of aluminum filter) int numRCF (number of RCF pieces), double maxPenergy (maximum proton energy for theoretical distribution) this number is recast into an the int maxProtEnergy so it can be used as an index, String fileName (filename of output data file).

2. init():: calls selected methods, essentially runs the program. only method called by driver TestProtStop.java.

3. makeFilmPack(): : build film pack, using the input variables.

Each layer is given a "Z" value that represents either its Atomic number or compound composition: Mylar $(\mathrm{Z}=93), \mathrm{RCF}$ dye $(\mathrm{Z}=94)$. creates filmPack[] where the first layer is filmPack[0]. There are three possible types of RCF. Each type is represented differently in the numRCF [] array.

$\mathrm{HS}=1$,

MD-55 $=2$,

$\mathrm{HD}-810=3$,

All have vrious composition parameters that are handled in the makeFilmPack() switch structure. Total size of filmPack[] = thickness of Al + thickness of each piece of RCF.

For reference of RCF: NIM A302, 165 (1991), or ISP web page www.ispcopr.com

4. stoppingcoef ()$:$ : method to read stopping coefficients from the Scoeff95A file from SRIM2000. Method builds 2-D double array (95 x 17) that will be used later to calculate energy deposited. First row is empty. There is no element $\mathrm{Z}=0$.

5. eDeposited():: creates energy deposited vs layer matrix. matrix defined as eLoss[energy][film-pack-layer]. Energies are numbered 0 to 999 , but correspond to $0.02 \mathrm{MeV}$ up to $20 \mathrm{MeV}$ in steps of $.02 \mathrm{MeV}$. 1000 energies in all. Second index depends on number of layers created in the makeFilmPack() method. 
6. sumEperLayer():: creates normalized sum of energies in each layer. Distribution is normalized to $N=(1 / \mathrm{kT}) \operatorname{Exp}(-$ Enot $/ \mathrm{kT})$. Energies are multiplied by this $N$ and then sumed for each layer. Generates array totalEperLayer [filmPack. length] .

7. ouputData():: writes data to file specified by String fileName declared in constructor or setVariables() method.

Record of revisions:

Date Programmer Description of change

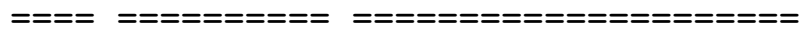

04/14/2003 matthew allen original code: filmpack method

04/16/2003 " added method to read Scoeff file

04/18/2003 " added energy deposition routine

04/25/2003 " added sum energy per layer method

09/03/2003 " modified to handle various forms of RCF

09/17/2003 " modified distribution function

09/23/2003 " Prav found my distribution problem

*/

package mallen.physics;

import java.io.*;

import chapman.io.*;

public class ProtonStopping \{

// Define class wide variables

int alThickness; //thickness of blast shield

public int blastSheildZ = 13; //atomic number of blast sheild

public int numRCF[]; //number of peices of RCF where index

//denotes type: HS=1, MD55=2, HD810=3.

public int filmPack[]; //declare film pack array.

String fileName; //name of output .DAT file.

int numRCFpieces; //number of pieces of film (type defined in MMA code). double maxPenergy; //maximum proton energy (may be double value).

int maxProtEnergy; //maximum proton energy (recast as integer in $\mathrm{keV}$ ).

String infile =

"C: /academic/laser-nuclear

/JanUSP/DTX/Stopping SRIM/Scoeff95Aadjusted.txt";

//location of SRIM Scoeff 


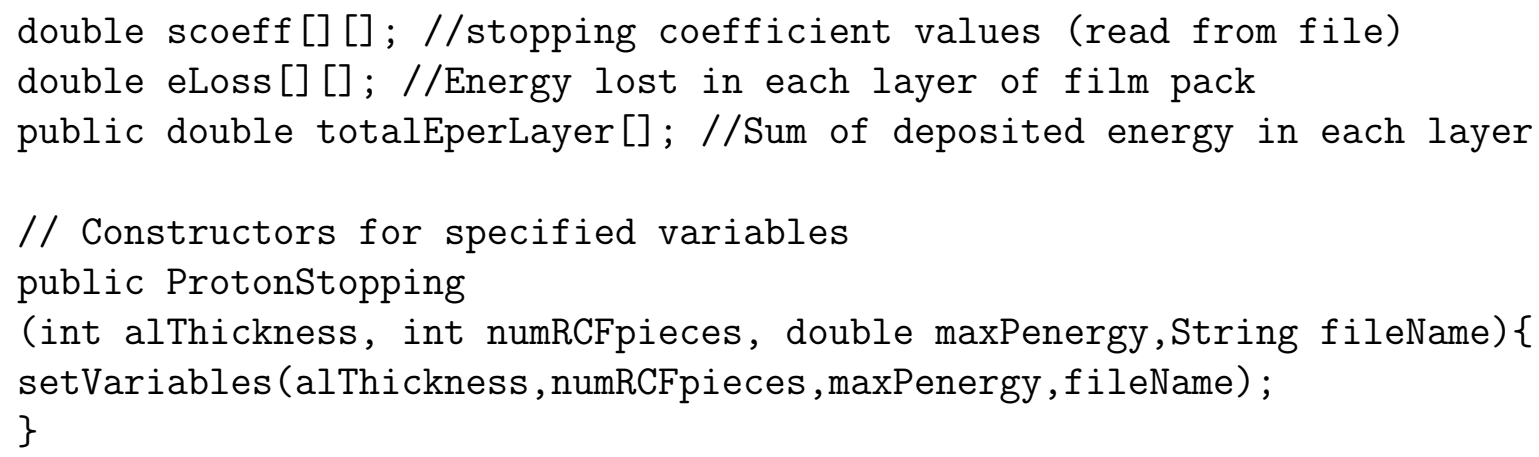


\} this.filmPack $=$ new int [packsize];

\}

// Initializing method

public void init() \{

makeFilmPack();

stoppingcoef ();

eDeposited ();

sumEperLayer (3.0);

//outputData(); //uncomment to write output file

\}

// Create filmpack array with each layer specified Z public void makeFilmPack()\{

//Define index to keep track of layer

int $\mathrm{n}=0$;

// variables for use in each case

int mylarThickness;

int dye;

int middleMylar;

//Create blast shield layer

for ( int $i=0 ; i<=$ alThickness; $i++)\{$

filmPack [i] =blastSheildZ;

\}

$\mathrm{n}=$ alThickness;

//Create sucessive layers of RCF

//begin switch structure for various types of RCF

for (int RCFi=0; RCFi < numRCF. length; RCFi++) \{

switch (numRCF [RCFi]) \{

$/ / \mathrm{RCF}=\mathrm{HS}$

case 1 :

mylarThickness $=97$;

dye $=40$;

//first mylar layer

for ( int $j=0 ; j<=$ mylarThickness; $j++$ ) \{ 


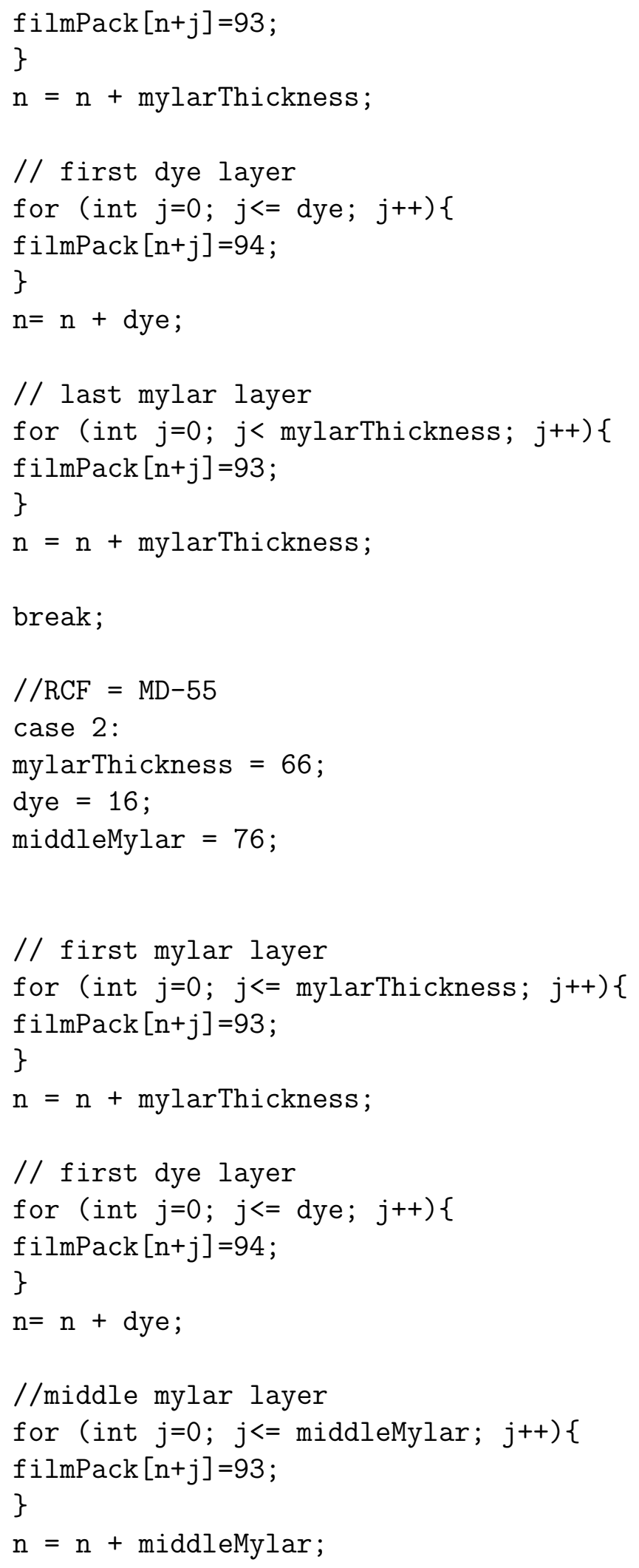


// second dye layer

for (int $j=0 ; j<=$ dye; $j++)\{$

filmPack $[n+j]=94$;

\}

$\mathrm{n}=\mathrm{n}+$ dye

// last mylar layer

for (int $j=0 ; j<$ mylarThickness; $j++$ ) \{

filmPack $[n+j]=93$;

\}

$\mathrm{n}=\mathrm{n}+$ mylarThickness;

break;

$/ / \mathrm{RCF}=\mathrm{HD}-810$

default:

dye $=6$;

mylarThickness $=97$;

// first dye layer

for (int $j=0 ; j<=$ dye; $j++)\{$

filmPack $[n+j]=94$;

\}

$\mathrm{n}=\mathrm{n}+$ dye;

// last mylar layer

for (int $j=0 ; j<$ mylarThickness; $j++$ ) \{

filmPack $[n+j]=93$;

\}

$\mathrm{n}=\mathrm{n}+$ mylarThickness;

break;

\}

\}

\}

public void stoppingcoef () \{

// Declare variables and arrays

int numElements $=92 ; / /$ number of elements and compounds

int numCoeff $=16 ; / /$ number of stopping values in file

this.scoeff $=$ new double[numElements +1$]$ [numCoeff +1$]$; 


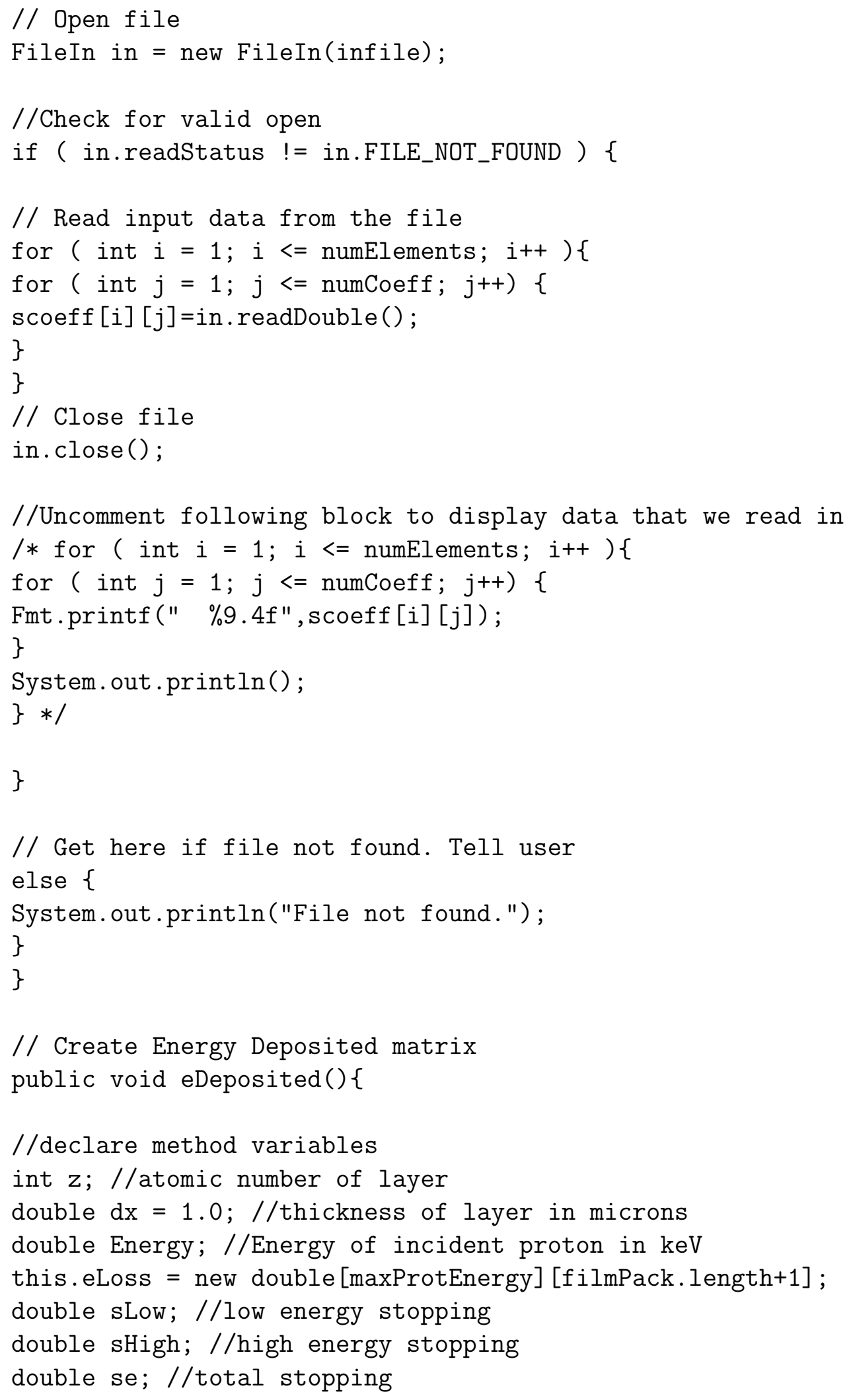


double se1,se2,se3, se4;//segmented stopping

//Nested loop to generate matrix

for (int Enot $=0$; Enot < maxProtEnergy; Enot ++ ) \{

Energy $=($ double $)$ Enot $* 20.0 ; / /$ incident energy in $\mathrm{keV}$

for (int $i=0 ; i<f i l m P a c k$.length; $i++)\{$

if (Energy $!=0)\{$.

$\operatorname{switch}(f i l m P a c k[i])\{$

// plastic layer $\mathrm{z}=93$

case 93:

$z=1$;

sLow $=$ scoeff $[z][9] *$ Math. pow (Energy, scoeff $[z][10])+\operatorname{scoeff}[z][11] *$

Math.pow(Energy, scoeff $[z][12])$;

sHigh=scoeff [z] [13] $*$ Math. $\log ((\operatorname{scoeff}[z][15] /$ Energy $)+(\operatorname{scoeff}[z][16] *$ Energy $))$ /Math.pow(Energy, scoeff [z] [14]);

se1 $=1.0 /(1.0 /$ sLow $+1.0 /$ sHigh $) ;$

$\mathrm{z}=6$;

sLow $=\operatorname{scoeff}[z][9] *$ Math. pow (Energy, scoeff $[z][10])+\operatorname{scoeff}[z][11] *$

Math.pow(Energy, scoeff $[z][12])$;

sHigh=scoeff $[z][13] *$ Math. $\log ((\operatorname{scoeff}[z][15] /$ Energy $)+(\operatorname{scoeff}[z][16] *$ Energy $))$ /Math.pow(Energy, scoeff [z] [14]);

se2 $=1.0 /(1.0 /$ sLow $+1.0 /$ sHigh $) ;$

$z=8$;

sLow $=\operatorname{scoeff}[z][9] *$ Math. pow (Energy, scoeff $[z][10])+\operatorname{scoeff}[z][11] *$

Math.pow(Energy, scoeff $[z][12])$;

sHigh=scoeff [z] [13] $*$ Math. $\log ((\operatorname{scoeff}[z][15] /$ Energy $)+(\operatorname{scoeff}[z][16] *$ Energy $))$ /Math.pow(Energy, scoeff $[z][14])$;

se3 $=1.0 /(1.0 /$ sLow $+1.0 /$ sHigh $) ;$

$\mathrm{se}=(4.0 * \operatorname{se} 1+5.0 * \operatorname{se} 2+2.0 * \operatorname{se} 3) / 11 . ;$ 


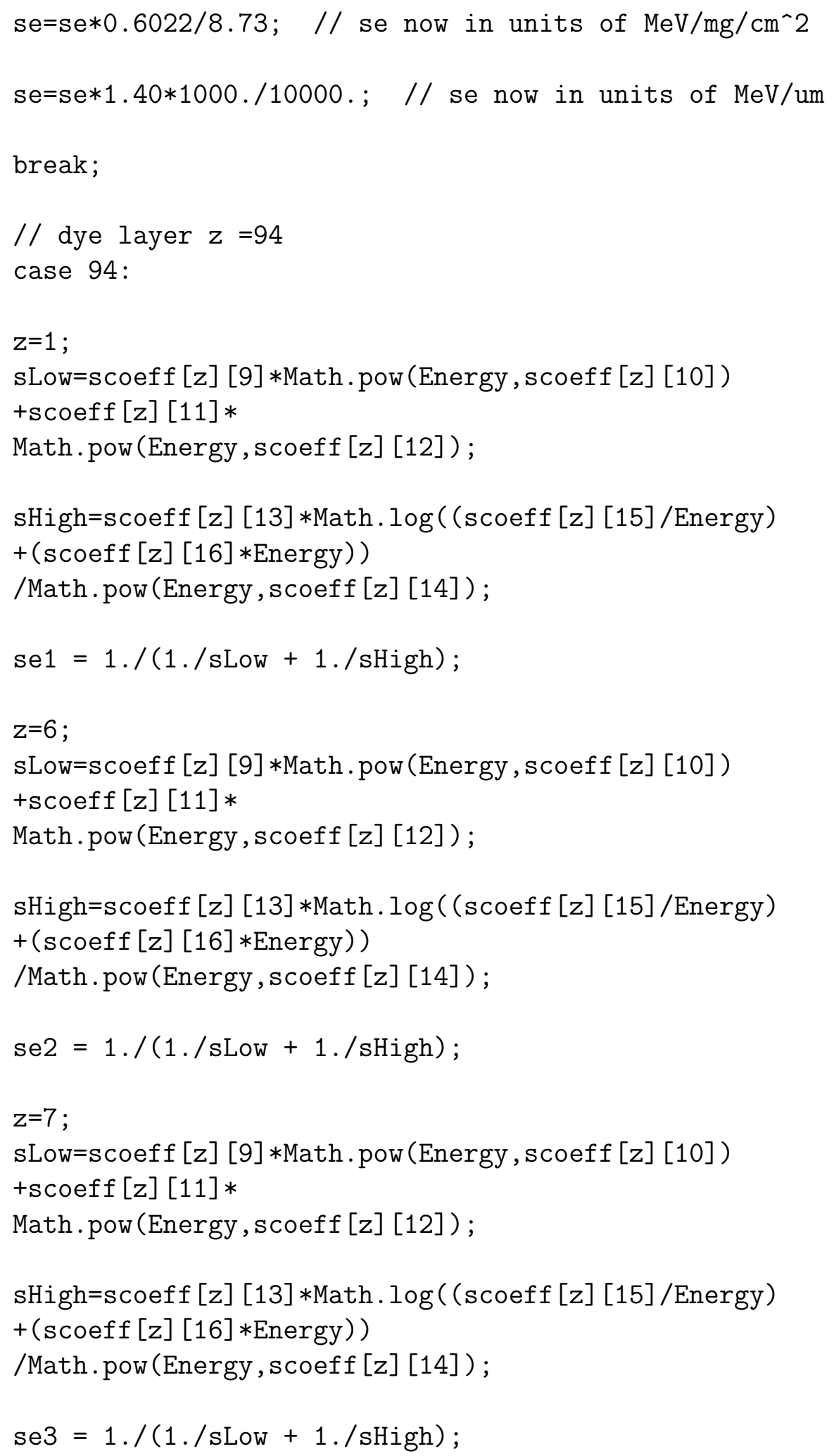


$\mathrm{z}=8$;

sLow $=$ scoeff $[z][9] *$ Math $\cdot$ pow (Energy, scoeff $[z][10])$

$+\operatorname{scoeff}[z][11] *$

Math.pow(Energy, scoeff $[z][12])$;

sHigh=scoeff $[z][13] *$ Math. $\log ((\operatorname{scoeff}[z][15] /$ Energy $)$

$+(\operatorname{scoeff}[z][16] *$ Energy $))$

/Math.pow(Energy, scoeff [z] [14]);

se4 $=1 . /(1 . /$ sLow $+1 . /$ sHigh $) ;$

$\mathrm{se}=(56.0 * \mathrm{se} 1+31.5 * \mathrm{se} 2+5.0 * \mathrm{se} 3+7.5 * \mathrm{se} 4) / 100 . ;$

$\mathrm{se}=\mathrm{se} * 0.6022 / 6.24 ; / /$ se now in units of $\mathrm{MeV} / \mathrm{mg} / \mathrm{cm}^{\wedge} 2$

se=se*1.09*1000./10000.; // se now in units of MeV/um

break;

default:

$z=f i l m P a c k[i]$;

sLow $=$ scoeff $[z][9] *$ Math $\cdot \operatorname{pow}($ Energy, scoeff $[z][10])$

$+\operatorname{scoeff}[z][11] *$

Math.pow(Energy, scoeff $[z][12])$;

sHigh $=$ scoeff $[z][13] *$ Math. $\log ((\operatorname{scoeff}[z][15] /$ Energy $)$

$+(\operatorname{scoeff}[z][16] *$ Energy $))$

/Math.pow(Energy, scoeff [z] [14]);

se $=1.0 /(1.0 /$ sLow $+1.0 /$ sHigh $)$;

se $=$ se $* 0.6022 / \operatorname{scoeff}[z][2] ; / /$ se now in units of $\mathrm{MeV} / \mathrm{mg} / \mathrm{cm}^{\wedge} 2$

se $=\operatorname{se} * \operatorname{scoeff}[z][5] *(1000 . / 10000.) ; / /$ se now in units of MeV/um break;

\}

Energy = Energy/1000.; // incident proton energy in units of MeV eLoss[Enot] [i]=se $* d x ; / /$ energy lost in units of $\mathrm{MeV}$

if $(($ Energy - eLoss [Enot] [i]) >0.) \{

eLoss[Enot][i]=se $* d x ; / /$ energy lost in units of $\mathrm{MeV}$

Energy $=($ Energy-eLoss [Enot] $[i]) * 1000 . ; / /$ reduced energy in keV

\}

else \{

eLoss [Enot] $[i]=$ Energy ; 


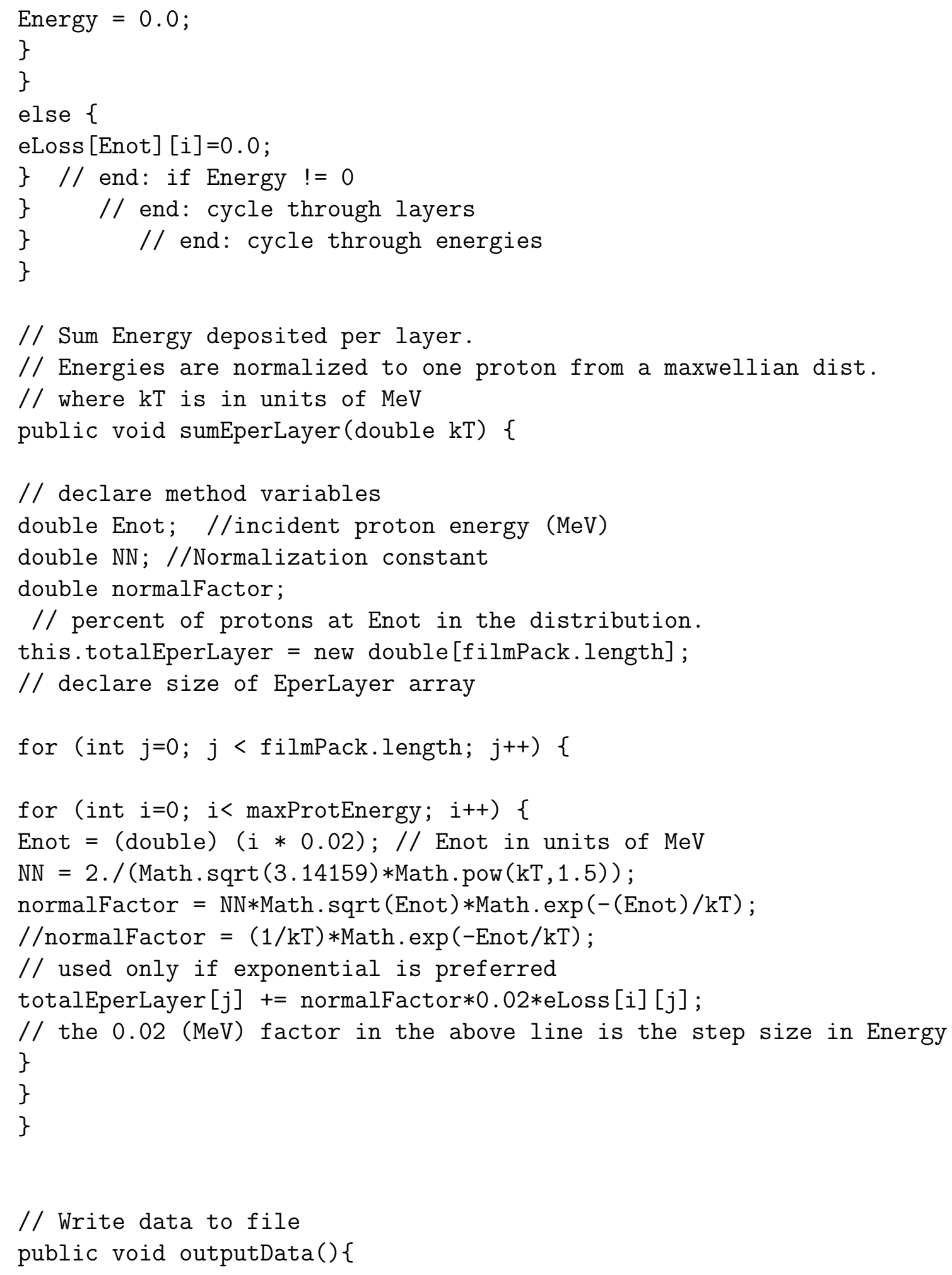




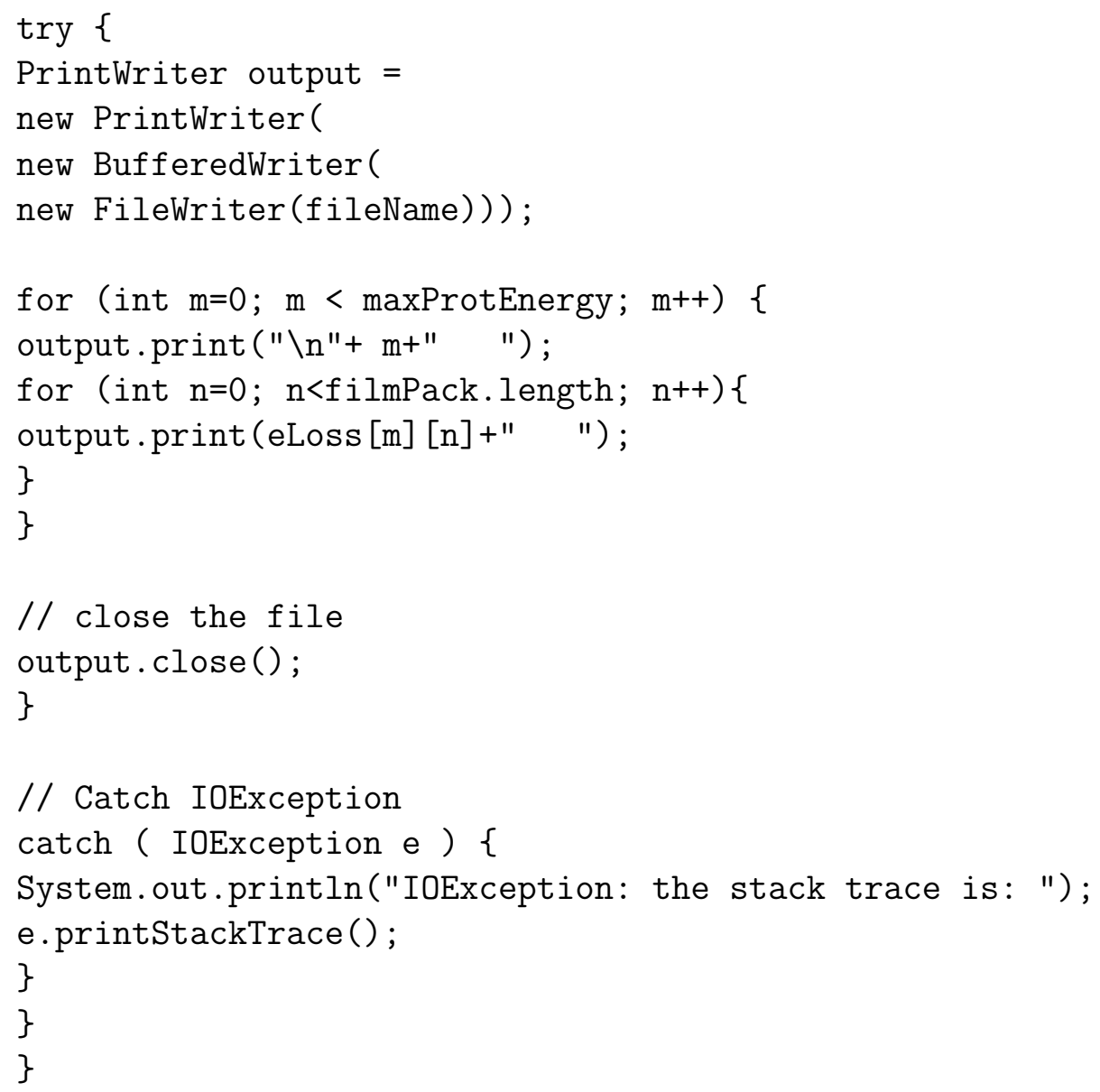




\section{Bibliography}

[1] F. F. Chen, Introduction to Plasma Physics and Controlled Fusion (Kluwer Academic Publishers, New York, 1984).

[2] J. Nuckolls, L. Wood, A. Theissen, and G. Zimmerman, Nature 239, 139 (1972).

[3] J. Lindl, Phys. Plasmas 2, 3933 (1995).

[4] M. D. Rosen, Phys. Plasmas 6, 1690 (1999).

[5] J. Meyer-ter-Vehn, Nucl. Fusion 22, 561 (1982).

[6] M. Tabak, J. Hammer, M. Glinsky, W. L. Kruer, S. C. Wilks, J. Woodworth, E. M. Campbell, and M. D. Perry, Phys. Plasmas 1, 1626 (1994).

[7] R. Kidder, Nucl. Fusion 16, 405 (1976).

[8] M. Roth, T. E. Cowan, M. H. Key, S. P. Hatchett, C. Brown, W. Fountain, J. Johnson, D. M. Pennington, R. A. Snavely, S. C. Wilks, K. Yasuike, H. Ruhl, F. Pegoraro, S. V. Bulanov, E. M. Campbell, M. D. Perry, and H. Powell, Phys. Rev. Lett. 86, 436 (2001). 
[9] M. D. Perry and G. Mourou, Science 264, 917 (1994).

[10] G. A. Mourou, C. P. J. Barty, and M. D. Perry, Physics Today 24, 398 (1998).

[11] D. Strickland and G. Mourou, Opt. Commun. 56, 219 (1985).

[12] D. E. Spence, P. N. Kean, and W. Sibbell, Opt. Lett. 16, 42 (1991).

[13] O. Svelto, Principles of Lasers (Plenum Press, New York, 1998).

[14] J. Kmetec, J. J. Macklin, and J. F. Young, Opt. Lett. 16, 1001 (1991).

[15] A. Sullivan et al., Opt. Lett. 16, 1406 (1991).

[16] P. Maine, D. Strickland, P. Bado, et al., IEEE J. Quant. Elec. 24, 398 (1988).

[17] M. Pessot, P. Maine, and G. Mourou, Opt. Commun. 62, 419 (1987).

[18] M. D. Perry, D. Pennington, and B. C. Stuart, Optics Lett. 24, 160 (1999).

[19] J. D. Bonlie, F. Patterson, D. Price, et al., Appl. Phys. B 70 Suppl., S155 (2000).

[20] A. Sullivan, J. D. Bonlie, D. Price, et al., Optics. Lett. 21, 603 (1996).

[21] F. Patterson, J. D. Bonlie, D. Price, et al., Optics. Lett. 24, 963 (1999).

[22] J. Itatani, J. Faure, M. Nantel, et al., Optics. Commun. 148, 70 (1998).

[23] S. C. Wilks, W. L. Kruer, M. Tabak, and A. B. Langdon, Phys. Rev. Lett. 69, $1383(1992)$. 
[24] S. C. Wilks and W. L. Kruer, IEEE J. Quant. Elec. 33, 1954 (1997).

[25] H. Hora, Laser Plasma Physics: forces and the nonlinearity principle (SPIE Press, Bellingham, WA, 2000).

[26] W. L. Kruer and S. C. Wilks, in Advances in Plasma Physics (AIP, New York, 1994), Chap. Introduction to Ultra-Intense Laser-Plasma Interactions, p. 16.

[27] S. C. Wilks, Phys. Fluids B 5, 2603 (1993).

[28] J. E. Allen and J. G. Andrews, J. Plasma Phys. 4, 187 (1970).

[29] J. Denavit, Phys. Fluids 22, 1384 (1979).

[30] F. Begay and D. W. Forslund, Phys. Fluids 25, 1675 (1982).

[31] S. J. Gitomer et al., Phys. Fluids 29, 2679 (1986).

[32] A. Macksimchuk, S. Gu, K. Flippo, et al., Phys. Rev. Lett. 84, 4108 (2000).

[33] E. Clark, K. Krushelnick, J. R. Davies, et al., Phys. Rev. Lett. 84, 670 (2000).

[34] S. Hatchett, C. G. Brown, T. E. Cowan, et al., Phys. Plasmas 7, 2076 (2000).

[35] R. A. Snavely, M. H. Key, S. P. Hatchett, et al., Phys. Rev. Lett. 85, 2945 (2000).

[36] K. Wharton, S. P. Hatchett, S. C. Wilks, et al., Phys. Rev. Lett. 81, 822 (1998).

[37] S. C. Wilks, A. B. Langdon, T. E. Cowan, et al., Phys. Plasmas 8, 542 (2001).

[38] A. Pukhov, Phys. Rev. Lett. 86, 3562 (2001). 
[39] I. S. Products, GAFCHROMIC Radiation Dosemitry Media, ISP homepage, 2003, http://www.ispcorp.com/products/dosimetry.

[40] N. V. Klassen and L. van der Zwan, Med. Phys. 24, 1924 (1997).

[41] J. Zeigler, The Stopping and Range of Ions in Matter, 2003, http://www.srim.org.

[42] J. Ziegler, Appl. Phys. Rev. 85, 1249 (1999).

[43] H. Andersen and J. Ziegler, Hydrogen Stopping Powers and Ranges in All Elements (Pergamon Press, New York, 1977), Vol. 3.

[44] H. W. Jr., R. Lanier, M. Mustafa, et al., Technical Report No. UCRL-ID-115738, Lawrence Livermore National Laboratory, Livermore, CA 94551 (unpublished).

[45] P. K. Patel, A. J. Mackinnon, D. F. Price, et al., Technical Report No. UCRLJC-143112, Lawrence Livermore National Laboratory, Livermore, CA 94551 (unpublished).

[46] B. Duane, Technical Report No. BNWL-1685, Brookhaven National Laboratory, Upton, NY 11973 (unpublished).

[47] M. Stoyer, T. Sangster, E. Henry, et al., Rev. Sci. Instr. 72, 767 (2001).

[48] M. Hegelich, S. Karsch, G. Pretzler, D. Habs, K. Witte, M. Allen, T. Cowan, P. Audebert, J. Fuchs, J. C. Gauthier, W. Guenther, A. Blasevicz, M. Geissel, and M. Roth, Phys. Rev. Lett. 89, 085002 (2002). 
[49] W. M. Mueller, J. P. Blackledge, and G. G. Libowitz, Metal Hydrides (Academic Press, New York, 1968).

[50] G. Alfred and J. Volkl, Hydrogen in Metals (Springer-Verlag, New York, 1978).

[51] M. Fleischmann and S. Pons, J. Electroanal. Chem. 261, 301 (1989).

[52] M. Fleischmann, S. Pons, and M. Hawkins, J. Electroanal. Chem. 263, 187 (1989).

[53] S. Jones, E. Palmer, J. Czirr, et al., Nature 338, 737 (1989).

[54] T. Ditmire, J. Zweiback, et al., Nature 398, 429 (1999).

[55] K. W. Madison, P. K. Patel, M. Allen, D. Price, and T. Ditmire, J. Opt. Soc. Amer. B 20, 113 (2003).

[56] A. J. Mackinnon, M. Borghesi, S. Hatchett, et al., Phys. Rev. Lett. 86, 1769 (2001).

[57] 3cm DC Source, \# 424945 rev. a ed., Veeco Instruments, 2002.

[58] IBS 250 Power Supply, \# 425193 rev. b ed., Veeco Instruments, 2002.

[59] M. Borghesi, A. Mackinnon, P. K. Patel, et al., Phy. Rev. Lett. (2004), in press.

[60] P. A. Thiel and T. E. Madey, Surf. Sci. Reports 7, 211 (1987).

[61] M. A. Henderson, Surf. Sci. Reports 46, 1 (2002). 
[62] A. B. Langdon and B. F. Lasinski, in Methods in Computational Physics (Academic, New York, 1976), Vol. 16, pp. 327-366, description of ZOHAR laserplasma PIC code.

[63] J. Scofield, private communication.

[64] P. K. Patel, A. J. Mackinnon, M. H. Key, M. E. Foord, M. Allen, D. F. Price, H. Ruhl, P. T. Springer, and R. Stephens, Phys. Rev. Lett. 91, 125004 (2003).

[65] R. Kodama, Nature 418, 933 (2002).

[66] R. B. Stephens, S. P. Hatchett, R. E. Turner, K. A. Tanaka, and R. Kodama, Phys. Rev. Lett. 91, 185001 (2003).

[67] K. Tanaka, R. Kodama, K. Mima, et al., Phys. Plasmas 10, 1925 (2003).

[68] J. Lennard-Jones, Trans. of the Faraday Soc. 28, 333 (1932).

[69] G. A. Somorjai and M. A. V. Hove, in Adsorbed Monolayers on Solid Surfaces, edited by J. D. Dunitz et al. (Springer-Verlag, New York, 1979), Vol. 38.

[70] I. Langmuir, Am. Chem. Soc. 40, 1361 (1918).

[71] J. Gottfried et al., Surf. Sci. 511, 65 (2002).

[72] R. I. Masel, Principles of Adsorption and Reaction on Solid Surfaces (John Wiley and Sons, New York, 1996), p. 208. 